\title{
Bordered Heegaard Floer homology and graph manifolds
}

\author{
JONATHAN HANSELMAN
}

\begin{abstract}
We perform two explicit computations of bordered Heegaard Floer invariants. The first is the type $D$ trimodule associated to the trivial $S^{1}$-bundle over the pair of pants $\mathcal{P}$. The second is a bimodule that is necessary for self-gluing when two torus boundary components of a bordered manifold are glued to each other. Using the results of these two computations, we describe an algorithm for computing $\widehat{\mathrm{HF}}$ of any graph manifold.
\end{abstract}

$57 \mathrm{M} 27,57 \mathrm{R} 58$

\section{Introduction}

Heegaard Floer homology is a collection of invariants for closed 3-manifolds introduced by Ozsváth and Szabó [27]. The package also contains invariants for 4-dimensional cobordisms and for knots and links; see Ozsváth and Szabó [28; 26], and Rasmussen [30]. It has proved to be a sensitive invariant, but in general, it is difficult to compute. The definition involves a chain complex whose generators are combinatorial but whose differential requires counting pseudoholomorphic curves.

There are a few existing algorithms for computing Heegaard Floer homology. Sarkar and Wang [31] developed a method using nice diagrams, for which computing the differential becomes combinatorial. This method has since been refined and extended; see Hales, Karabash and Lock [3], and Ozsváth, Stipsicz and Szabó [20; 21]. Another approach uses grid diagrams and surgery formulas; see Manolescu, Ozsváth and Sarkar [14], Manolescu and Ozsváth [13], and Manolescu, Ozsváth and Thurston [15]. These algorithms greatly increase the number of generators and, as a result, are not practical for large manifolds. A third algorithm is based on computing the bordered Heegaard Floer invariant for the surface diffeomorphism associated with a Heegaard splitting; see Lipshitz, Ozsváth and Thurston [9]. This algorithm has a computer implementation which is practical for splittings up to genus 3 . However, efficiently computing Heegaard Floer homology for general 3-manifolds remains a difficult problem.

If we restrict to particular classes of 3-manifolds, computing Heegaard Floer homology becomes easier. For example, much is known about the Heegaard Floer homology 
of manifolds which are obtained by plumbing circle bundles according to a negative definite tree $\Gamma$. Ozsváth and Szabó [25] gave a combinatorial description of $\mathrm{HF}^{+}$ of these manifolds when the tree $\Gamma$ has at most one "bad" vertex. This class of manifolds includes all Seifert fibered rational homology spheres. Their algorithm for computing $\mathrm{HF}^{+}$has been useful, for instance, in determining the existence of tight contact structures on Seifert fibered spaces; see Lisca and Stipsicz [12]. Némethi [17] introduced an invariant for negative definite plumbings, lattice homology, which is combinatorially computable and conjecturally equivalent to $\mathrm{HF}^{+}$. Recent work has explored this conjectured equivalence; there is a spectral sequence from lattice cohomology to $\mathrm{HF}^{+}$, and they are known to be isomorphic for plumbings with at most two bad vertices; see Ozsváth, Stipsicz and Szabó [22; 23; 24].

There has been significant interest in understanding $L$-spaces (manifolds with minimal Heegaard Floer homology) and the relationship between this condition and the existence of taut foliations and left orderability of the fundamental group. The conjectured relationship between these conditions is known to hold for particular classes of 3manifolds, including Seifert fibered manifolds; see Peters [29], and Boyer, Gordon and Watson [2]. These geometric conditions can help us determine the $L$-space condition of a manifold even if we cannot compute $\widehat{\mathrm{HF}}$ directly. Mauricio [16] used lattice homology and the existence of taut foliations to give sufficient conditions on the weights of a negative definite tree $\Gamma$ under which a plumbing is or is not an $L$-space.

The plumbings of negative definite trees mentioned above are special cases of graph manifolds. A graph manifold is a 3-manifold whose JSJ decomposition contains only Seifert fibered pieces. The non-Seifert fibered pieces in a JSJ decomposition are hyperbolic, so with respect to geometrization, a graph manifold is a manifold with no hyperbolic pieces in its geometric decomposition. Thus graph manifolds represent an important subclass of 3-manifolds. For a brief overview of graph manifolds and their place in 3-manifold topology, see Neumann [19]. In this paper, we present a method for computing $\widehat{\mathrm{HF}}$ for any graph manifold, which is based on computing bordered Heegaard Floer invariants for certain fundamental building blocks from which graph manifolds can be constructed.

This method finds a middle ground between the approaches mentioned above. It is more general than results restricted to negative definite plumbing trees, since it works for arbitrary graph manifolds. At the same time, it is more computationally practical than current algorithms for general 3-manifolds. There is a computer implementation of this algorithm that is capable of handling quite complicated manifolds; it can been used, for instance, to show that the rank of $\widehat{\mathrm{HF}}$ of the graph manifold represented by the weighted tree in Figure 24 is 213,312. 
Let us recall some important facts and terminology concerning graph manifolds, following the notation found in Neumann [18]. A graph manifold can be encoded by a decorated graph:

Definition 1.1 A connected closed plumbing graph is a finite connected graph $\Gamma$ decorated as follows:

- $\quad$ each vertex $i$ carries two integer weights, $g_{i}$ and $e_{i}$;

- each edge carries a sign, + or - .

We allow $\Gamma$ to have multiple edges connecting two vertices or edges connecting a vertex to itself.

A connected closed plumbing graph $\Gamma$ specifies a (prime) graph manifold $M(\Gamma)$ as follows: For each vertex $i$ of $\Gamma$, let $d_{i}$ be the degree of the vertex. Let $F_{i}$ be the compact surface of genus $g_{i}$ with $d_{i}$ boundary components, where if $g_{i}<0$ we mean that $F_{i}$ is nonorientable of genus $\left|g_{i}\right|$. Let $E_{i}$ be the circle bundle with orientable total space over $F_{i}$ with a chosen trivialization on the boundary and Euler number $e_{i}$ (the Euler number is well defined once the trivialization on the boundary is chosen). The edges of $\Gamma$ dictate how the $E_{i}$ are glued together. For each edge connecting vertices $i$ and $j$, a component $S^{1} \times S^{1}$ of $\partial E_{i}$ is glued to a component $S^{1} \times S^{1}$ of $\partial E_{j}$. The gluing always exchanges base and fiber directions; for $(+)$-edges the gluing map is $\left(\begin{array}{ll}0 & 1 \\ 1 & 0\end{array}\right)$, and for $(-)$-edges the gluing map is $\left(\begin{array}{rr}0 & -1 \\ -1 & 0\end{array}\right)$. In either case, the gluing map is orientation reversing, and so $M(\Gamma)$ inherits consistent orientations from all of the $E_{i}$. For each edge connecting a vertex $i$ to itself, two components of $\partial E_{i}$ are glued with the appropriate gluing map.

Every prime graph manifold can be represented by a connected closed plumbing graph (for nonprime graph manifolds we allow disconnected graphs). The representation is not unique, but [18, Section 4] gives a well developed calculus for manipulating plumbing graphs. In particular, plumbing graphs can be reduced to a normal form, and graphs of this form represent manifolds uniquely. A few additional facts are worth mentioning here:

- Often changing the sign of an edge does not change the resulting 3-manifold. In fact, all that matters is the total sign around each loop in $\Gamma$. In particular, for acyclic graphs we may ignore the decoration on the edges.

- It is possible to represent any graph manifold with a plumbing graph such that no vertex is assigned a negative genus. 
- We can describe graph manifolds with boundary by adding an additional weight $b_{i}$ to each vertex $i$. In the construction, $F_{i}$ is the genus $g_{i}$ surface with $b_{i}+d_{i}$ boundary components. $E_{i}$ is the appropriate circle bundle over $F_{i}$, and $b_{i}$ components of $\partial E_{i}$ are not glued to anything.

Bordered Heegaard Floer homology is an extension of Heegaard Floer homology to manifolds with boundary; see Lipshitz, Ozsváth and Thurston [8]. Because graph manifolds decompose so nicely, bordered Heegaard Floer homology provides a natural approach for computing their $\widehat{\mathrm{HF}}$. The key ingredient is to compute the bordered invariants for arbitrary $S^{1}$-bundles over surfaces, the building blocks of graph manifolds. Changing the Euler number of one of these bundles is equivalent to changing the parametrization of the boundary, which can be accomplished by tensoring with a well understood bimodule; see Lipshitz, Ozsváth and Thurston [10, Section 10.2]. As a result, we only need to compute invariants for trivial bundles over surfaces. As noted above, it is sufficient to consider bundles over orientable surfaces. Furthermore, any orientable surface has a pants decomposition - it can be obtained by gluing together copies of the pair of pants $\mathcal{P}=S^{3} \backslash$ three open disks\}. The trivial $S^{1}$-bundle over the surface can be obtained by gluing copies of the trivial $S^{1}$-bundle over $\mathcal{P}$. Thus we see that the trivial bundle $\mathcal{Y}_{\mathcal{P}}=\mathcal{P} \times S^{1}$ is the fundamental building block for graph manifolds.

In Section 2 we will review the relevant background from bordered Heegaard Floer homology. The trimodule $\widehat{\mathrm{CFD}}^{3}\left(\mathcal{Y}_{\mathcal{P}}\right)$ will be explicitly computed in Section 3, proving the following:

Theorem 1.2 The summand of the type $D$ trimodule $\widehat{\mathrm{CFD}}^{3}\left(\mathcal{Y}_{\mathcal{P}}\right)$ in the middle spinc ${ }^{-}$ structure has five generators as a projective module: $v, w, x, y$ and $z$. Up to quasiisomorphism, the differential is given by the following diagram:

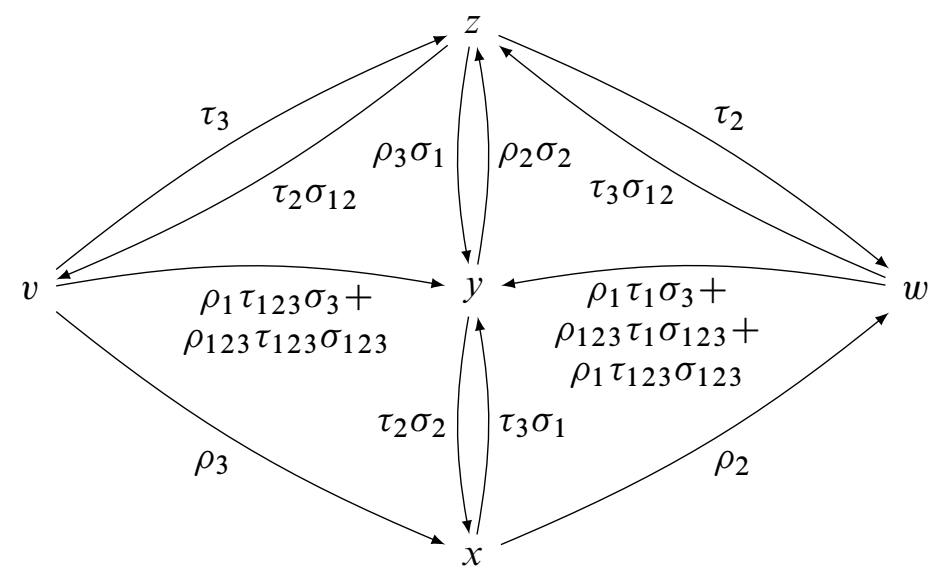


$\widehat{\mathrm{CFD}}^{3}\left(\mathcal{Y}_{\mathcal{P}}\right)$ in the other $\operatorname{spin}^{c}$-structures will also be computed. For acyclic plumbing graphs with only genus- 0 vertices, $\widehat{\mathrm{HF}}$ of the corresponding graph manifold can be obtained from the trimodule in Theorem 1.2 and bimodules for mapping classes of the torus. If the graph has a cycle or some vertex has genus $g_{i}>0$, then an additional bimodule is needed. A bordered Heegaard diagram for this bimodule was described by Lipshitz and Treumann in [11, Section 4.4], but the bimodule was not computed. In Section 4 we explicitly compute this bimodule, using the bordered Heegaard diagram $\mathcal{H}_{\mathrm{SG}}$ in Figure 15.

Theorem 1.3 The bimodule $\widehat{\mathrm{CFDD}}\left(\mathcal{H}_{\mathrm{SG}}\right)$ in the middle spin $^{c}$-structure is given by Figure 20. In the extremal spin ${ }^{c}-$ structures, it is quasi-isomorphic to zero.

Finally, given an arbitrary plumbing graph, Section 5 will describe the procedure for piecing together the relevant bordered invariants to obtain $\widehat{\mathrm{HF}}$ of the corresponding graph manifold.

Acknowledgments I would like to thank Robert Lipshitz for suggesting this problem and for many helpful conversations about these computations. I am also grateful to Adam Levine, Peter Ozsváth, Dylan Thurston and Liam Watson for helpful conversations. I especially thank Adam Levine for suggesting a finger move isotopy which simplified the computation in Section 3. This work partially supported by NSF Research Training Groups grant number DMS-0739392.

\section{Background on Heegaard Floer homology}

We begin by recalling the essential definitions and properties concerning the bordered Heegaard Floer invariants developed by Lipshitz, Ozsváth and Thurston. For a full treatment of these invariants, see $[8 ; 10]$. We discuss only the details that will be needed in the rest of the paper. In particular, we restrict to the case of manifolds with toroidal boundary components, which simplifies many of the definitions.

\section{A Algebraic definitions}

Let $(\mathcal{A}, d)$ be a unital differential algebra over $\mathbb{F}_{2}$, with a subring of idempotents $\mathcal{I}$, and let $\left\{\iota_{i}\right\}$ be the indecomposable idempotents, with $\mathbf{1}=\sum \iota_{i}$.

A (left) type $D$ structure over $\mathcal{A}$ is a vector space $N$ over $\mathbb{F}_{2}$ with a left action of $\mathcal{I}$ and a map

$$
\delta_{1}: N \rightarrow \mathcal{A} \otimes_{\mathcal{I}} N
$$

satisfying the relation

$$
\left(\mu \otimes \mathrm{id}_{N}\right) \circ\left(\mathrm{id}_{\mathcal{A}} \otimes \delta_{1}\right) \circ \delta_{1}+\left(d \otimes \mathrm{id}_{N}\right) \circ \delta_{1}=0,
$$


where $\mu: \mathcal{A} \otimes \mathcal{A} \rightarrow \mathcal{A}$ is multiplication on $\mathcal{A}$. The tensor product $\mathcal{A} \otimes_{\mathcal{I}} N$ is a left differential $\mathcal{A}$ module, with module structure $a \cdot(b \otimes x)=a b \otimes x$ and differential given by $\partial(a \otimes x)=a \cdot \delta_{1}(x)+d(a) \otimes x$. The relation (1) ensures that $\partial^{2}=0$. Given the map $\delta_{1}$, define

$$
\delta_{k}: N \rightarrow \underbrace{\mathcal{A} \otimes_{\mathcal{I}} \cdots \otimes_{\mathcal{I}} \mathcal{A}}_{k \text { times }} \otimes_{\mathcal{I}} N
$$

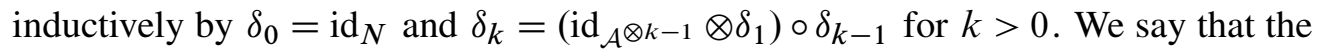
type $D$ structure $N$ is bounded if $\delta_{k}=0$ for all $k$ sufficiently large.

We will need to work with modules with multiple left actions. Let $\mathcal{A}_{1}, \ldots, \mathcal{A}_{k}$ be differential algebras, with rings of idempotents $\mathcal{I}_{1}, \ldots, \mathcal{I}_{k}$. A $k$-fold type $D$ structure over $\mathcal{A}_{1}, \ldots, \mathcal{A}_{k}$ is a type $D$ structure over $\mathcal{A}_{1} \otimes \cdots \otimes \mathcal{A}_{k}$. We will call the module $\left(\mathcal{A}_{1} \otimes \cdots \otimes \mathcal{A}_{k}\right) \otimes_{\left(\mathcal{I}_{1} \otimes \cdots \otimes \mathcal{I}_{k}\right)} N$ a type $D$ multimodule over $\mathcal{A}$.

A (right) $\mathcal{A}_{\infty}$ module (or type $A$ structure) over $\mathcal{A}$ is a vector space $M$ over $\mathbb{F}_{2}$ with a right action of $\mathcal{I}$ and maps

$$
m_{k+1}: M \otimes_{\mathcal{I}} \underbrace{\mathcal{A} \otimes_{\mathcal{I}} \cdots \otimes_{\mathcal{I}} \mathcal{A}}_{k \text { times }} \rightarrow M
$$

satisfying the following $\mathcal{A}_{\infty}$ relation for any $x \in M$ and any $a_{1}, \ldots, a_{n} \in \mathcal{A}$ :

$$
\begin{aligned}
0=\sum_{i=0}^{n} m_{n-i+1}\left(m_{i+1}\left(x, a_{1}, \ldots, a_{i}\right), a_{i+1}, \ldots, a_{n}\right) \\
+\sum_{i=1}^{n-1} m_{n}\left(x, a_{1}, \ldots, a_{i-1}, \mu\left(a_{i}, a_{i+1}\right), a_{i+2}, \ldots, a_{n}\right) \\
+\sum_{i=1}^{n} m_{n+1}\left(x, a_{1}, \ldots, a_{i-1}, d\left(a_{i}\right), a_{i+1}, \ldots, a_{n}\right) .
\end{aligned}
$$

An informal statement of the $\mathcal{A}_{\infty}$ relations may be easier to remember: for any ordered set of inputs, the sum of all ways of combining those inputs using two steps (where each step is $\mu, d$ or some $\left.m_{i}\right)$ is zero. We also require that $m_{2}(x, 1)=x$ and $m_{k}(x, \ldots, \mathbf{1}, \ldots)=0$ for all $k>2$. If $m_{k}=0$ for all sufficiently large $k$, we say that the $\mathcal{A}_{\infty}$ module $M$ is bounded.

More generally, we can define an $\mathcal{A}_{\infty}$ multimodule $M$ over $\mathcal{A}_{1}, \ldots, \mathcal{A}_{k}$ as follows: $M$ is a vector space over $\mathbb{F}_{2}$ with a right action of $\mathcal{I}_{1} \otimes \cdots \otimes \mathcal{I}_{k}$. Also, $M$ is equipped with maps

$$
m_{1, i_{1}, \ldots, i_{k}}: M \otimes_{\mathcal{I}_{1} \otimes \cdots \otimes \mathcal{I}_{k}} \mathcal{A}_{1}^{\otimes i_{1}} \otimes \cdots \otimes \mathcal{A}_{k}^{\otimes i_{k}} \rightarrow M
$$

satisfying an appropriate version of the $\mathcal{A}_{\infty}$ relation (we will generally suppress the subscripts on $m$ from the notation). To define the relation, we introduce the following 
functions. For $\vec{a}_{\ell}=\left(a_{\ell}^{1}, \ldots, a_{\ell}^{k}\right) \in \mathcal{A}_{\ell}^{\otimes k}$ and $0 \leq j \leq k$, define

$$
\begin{aligned}
T_{j}\left(\vec{a}_{\ell}\right) & :=\left(a_{\ell}^{1}, \ldots, a_{\ell}^{j}\right) \in \mathcal{A}_{\ell}^{\otimes j} \\
T^{j}\left(\vec{a}_{\ell}\right) & :=\left(a_{\ell}^{j+1}, \ldots, a_{\ell}^{k}\right) \in \mathcal{A}_{\ell}^{\otimes k-j} \\
\bar{\mu}\left(\vec{a}_{\ell}\right) & :=\sum_{j=1}^{k-1}\left(a_{\ell}^{1}, \ldots, a_{\ell}^{j-1}, a_{\ell}^{j} a_{\ell}^{j+1}, a_{\ell}^{j+2}, \ldots, a_{\ell}^{k}\right) \in \mathcal{A}_{\ell}^{\otimes k-1}, \\
\bar{d}\left(\vec{a}_{\ell}\right) & :=\sum_{j=1}^{k}\left(a_{\ell}^{1}, \ldots, a_{\ell}^{j-1}, d\left(a_{\ell}^{j}\right), a_{\ell}^{j+1}, \ldots, a_{\ell}^{k}\right) \in \mathcal{A}_{\ell}^{\otimes k} .
\end{aligned}
$$

Now we can write down the $\mathcal{A}_{\infty}$ relation for multimodules. For any $x \in M$ and any $\vec{a}_{1}, \ldots, \vec{a}_{k}$ in $\mathcal{A}^{\otimes i_{1}}, \ldots, \mathcal{A}^{\otimes i_{k}}$, we have

$$
\begin{aligned}
0=\sum_{j_{1}, \ldots, j_{k}} m\left(m\left(x, T_{j_{1}}\left(\vec{a}_{1}\right), \ldots, T_{j_{k}}\left(\vec{a}_{k}\right)\right), T^{j_{1}}\left(\vec{a}_{1}\right), \ldots, T^{j_{k}}\left(\vec{a}_{k}\right)\right) \\
+\sum_{j=1}^{k} m\left(x, \vec{a}_{1}, \ldots, \vec{a}_{j-1}, \bar{\mu}\left(\vec{a}_{j}\right), \vec{a}_{j+1}, \ldots, \vec{a}_{k}\right) \\
+\sum_{j=1}^{k} m\left(x, \vec{a}_{1}, \ldots, \vec{a}_{j-1}, \bar{d}\left(\vec{a}_{j}\right), \vec{a}_{j+1}, \ldots, \vec{a}_{k}\right) .
\end{aligned}
$$

It is possible to define combination multimodules, with some type $D$ actions and some type $A$ actions. Such a multimodule $N$ is equipped with maps

$$
\delta_{1}^{1, i_{k+1}, \ldots, i_{\ell}}: N \otimes \mathcal{A}_{1}^{\otimes i_{k+1}} \otimes \cdots \otimes \mathcal{A}_{k}^{\otimes i_{\ell}} \rightarrow \mathcal{A}_{k+1} \otimes \cdots \otimes \mathcal{A}_{k+\ell} \otimes N
$$

satisfying the appropriate versions of (1) and (3). Type $D D, A A$ and $D A$ bimodules are discussed in [10], and the generalization to more algebra actions is straightforward.

If $M$ is an $\mathcal{A}_{\infty}$ module over $\mathcal{A}$ and $N$ is a type $D$ module over $\mathcal{A}$, and if at least one of them is bounded, we may define the box tensor product $M \otimes N$ to be the vector space $M \otimes_{\mathcal{I}} N$ equipped with the differential

$$
\partial^{\bigotimes}(x \otimes y)=\sum_{k=0}^{\infty}\left(m_{k+1} \otimes \operatorname{id}_{N}\right)\left(x \otimes \delta_{k}(y)\right) .
$$

If $M$ is a multimodule over $\mathcal{A}_{1}, \ldots, \mathcal{A}_{k}$ such that the action of $\mathcal{A}_{k}$ is type $A$, and $N$ is a multimodule over $\mathcal{A}_{k}, \mathcal{A}_{k+1}, \ldots, \mathcal{A}_{k+\ell}$ such that the action of $\mathcal{A}_{k}$ is type $D$, and either $M$ or $N$ is bounded, then a box tensor product with respect to $\mathcal{A}_{k}$ can be defined in a similar way (see [10, Section 2.3.2] for the case when $N$ and $M$ are bimodules). $M \otimes_{\mathcal{A}_{k}} N$ is a multimodule over $\mathcal{A}_{1}, \ldots, \mathcal{A}_{k-1}, \mathcal{A}_{k+1}, \ldots, \mathcal{A}_{k+l}$, and 
the operations on $M \otimes N$ are determined by pairing operations on $M$ with sequences of operations in $N$ such that the $A_{k}$ outputs of the operations on $N$ match the $A_{k}$ inputs of the operation on $M$.

Remark 2.1 We will often represent a $k$-fold type $D$ multimodule $M$ as a labeled, directed graph, where vertices correspond to the generators of $M$, and there is an arrow from $x_{i}$ to $x_{j}$ labeled by $a_{i j}$ if $a_{i j} \neq 0$ is the coefficient of $x_{j}$ in $\partial\left(x_{i}\right)$. Here $a_{i j}$ is an element of $\mathcal{A}_{1} \otimes \cdots \otimes \mathcal{A}_{k}$, the tensor product of $k$ copies of the torus algebra. We omit the edge label when $a_{i j}=1$. We sometimes refer to an unlabeled arrow from $x_{i}$ to $x_{j}$ as a differential from $x_{i}$ to $x_{j}$. Graphs with unlabeled edges can be simplified by a well known edge reduction algorithm [6, Section 2.6]: we eliminate the endpoints $x_{i}$ and $x_{j}$ of the unlabeled edge and all edges attached to these two vertices, and for each "zig-zag"

$$
x_{k} \stackrel{a_{k j}}{\longrightarrow} x_{j} \longleftarrow x_{i} \stackrel{a_{i \ell}}{\longrightarrow} x_{\ell}
$$

we add an edge

$$
x_{k} \stackrel{a_{k j} a_{i \ell}}{\longrightarrow} x_{\ell},
$$

or if there is already an edge from $x_{k}$ to $x_{\ell}$ we add $a_{k j} a_{i \ell}$ to the label of that edge. The resulting graph represents a type $D$ multimodule that is quasi-isomorphic to $M$.

\section{B The torus algebra}

To define bordered Heegaard Floer invariants, we associate a differential algebra to each boundary component of a 3-manifold with boundary. The algebra associated to the torus splits into a direct sum

$$
\mathcal{A}\left(T^{2}\right)=\mathcal{A}\left(T^{2},-1\right) \oplus \mathcal{A}\left(T^{2}, 0\right) \oplus \mathcal{A}\left(T^{2}, 1\right) .
$$

$\mathcal{A}\left(T^{2},-1\right)$ is $\mathbb{F}_{2}$, and $\mathcal{A}\left(T^{2}, 1\right)$ is quasi-isomorphic to $\mathbb{F}_{2}$, so we need only discuss $\mathcal{A}\left(T^{2}, 0\right)$.

The algebra $\mathcal{A}\left(T^{2}, 0\right)$ is generated as a vector space over $\mathbb{F}_{2}$ by eight elements: two idempotents, $\iota_{0}$ and $\iota_{1}$, and six Reeb elements $\rho_{1}, \rho_{2}, \rho_{3}, \rho_{12}, \rho_{23}$ and $\rho_{123}$. The idempotents satisfy $\iota_{i} \iota_{j}=\delta_{i j} \iota_{i}$, and the identity element is $\mathbf{1}=\iota_{0}+\iota_{1}$. The Reeb elements interact with idempotents on either side as follows:

$$
\begin{array}{cl}
\iota_{0} \rho_{1}=\rho_{1} \iota_{1}=\rho_{1}, \quad \iota_{1} \rho_{2}=\rho_{2} \iota_{0}=\rho_{2}, & \iota_{0} \rho_{3}=\rho_{3} \iota_{1}=\rho_{3}, \\
\iota_{0} \rho_{12}=\rho_{12} \iota_{0}=\rho_{12}, \quad \iota_{1} \rho_{23}=\rho_{23} \iota_{1}=\rho_{23}, & \iota_{0} \rho_{123}=\rho_{123} \iota_{1}=\rho_{123} .
\end{array}
$$

The only nonzero products of Reeb elements are $\rho_{1} \rho_{2}=\rho_{12}, \rho_{2} \rho_{3}=\rho_{23}$ and $\rho_{1} \rho_{23}=\rho_{12} \rho_{3}=\rho_{123}$. Although $\mathcal{A}\left(T^{2}\right)$ is a differential algebra, the differential on $\mathcal{A}\left(T^{2}, 0\right)$ is zero. For more on the torus algebra and how it arises in bordered Heegaard Floer homology, see [8, Section 11.1]. 


\section{C Bordered manifolds and bordered diagrams}

A bordered 3-manifold with $k$ torus boundary components is an oriented 3-manifold $Y$, where $\partial Y$ is a disjoint union of $k$ tori $F_{1}, \ldots, F_{k}$, along with diffeomorphisms $\phi_{i}: T^{2} \rightarrow F_{i}$. If $\phi_{i}$ is orientation reversing, then the corresponding boundary component is said to be type $D$; otherwise it is said to be type $A$. In this paper, we will deal almost exclusively with type $D$ boundaries.

A bordered 3-manifold can be represented by an arced bordered Heegaard diagram.

Definition 2.2 An arced bordered Heegaard diagram with $k$ (torus) boundary components is a quadruple $(\Sigma, \boldsymbol{\alpha}, \boldsymbol{\beta}, \boldsymbol{z})$, where

- $\quad \Sigma$ is a compact surface of genus $g$ with $k$ boundary components;

- $\boldsymbol{\alpha}=\left\{\alpha_{1}^{1}, \alpha_{2}^{1}, \alpha_{1}^{2}, \alpha_{2}^{2}, \ldots, \alpha_{1}^{k}, \alpha_{2}^{k}, \alpha_{1}, \alpha_{2}, \ldots, \alpha_{g-k}\right\}$, where $\alpha_{1}^{i}$ and $\alpha_{2}^{i}$ are arcs embedded in $\Sigma$ with boundary on the $i^{\text {th }}$ component of $\partial \Sigma, \alpha_{j}$ is an embedded circle in $\Sigma$, and the $\alpha$ circles/arcs are pairwise disjoint;

- $\quad \boldsymbol{\beta}$ is $g$-tuple of disjoint circles in $\Sigma$;

- $z$ is a basepoint $z$ in $\Sigma \backslash(\boldsymbol{\alpha} \cup \boldsymbol{\beta})$ together with $\operatorname{arcs}$ in $\Sigma \backslash(\boldsymbol{\alpha} \cup \boldsymbol{\beta})$ connecting $z$ to each boundary component of $\Sigma$.

We also require that $\boldsymbol{\alpha}$ and $\boldsymbol{\beta}$ intersect transversely and $\Sigma \backslash \boldsymbol{\alpha}$ and $\Sigma \backslash \boldsymbol{\beta}$ are connected.

An arced bordered Heegaard diagram gives rise to a bordered 3-manifold by attaching $2-$ handles to a thickened version of the Heegaard surface $\Sigma$. The one and two boundary cases are described in Constructions 5.3 and 5.6 of [10], and the construction for more boundary components is completely analogous.

To define bordered invariants, we will also need to equip a bordered Heegaard diagram with labels on the boundary, as in Figure 1. Each component of $\partial \Sigma$ is divided into four segments by the $\operatorname{arcs} \alpha_{1}^{i}$ and $\alpha_{2}^{i}$, with one containing a basepoint, an endpoint of an arc in $\boldsymbol{z}$. Progressing from the basepointed segment in the direction which agrees with the boundary orientation on $\partial \Sigma$, we label the three remaining segments on the $i^{\text {th }}$ boundary component by $\rho_{1}^{i}, \rho_{2}^{i}$ and $\rho_{3}^{i}$ for type $A$ boundaries, or by $\rho_{3}^{i}, \rho_{2}^{i}$ and $\rho_{1}^{i}$ for type $D$ boundaries. In each case, $\rho_{12}^{i}, \rho_{23}^{i}$ and $\rho_{123}^{i}$ refer to the relevant concatenations. We call these oriented arcs Reeb chords. The assumption that $\Sigma \backslash \alpha$ is connected implies that the endpoints of $\alpha_{1}^{i}$ and $\alpha_{2}^{i}$ alternate. We assume that the first endpoint after the basepoint (following the boundary orientation) is $\alpha_{1}^{i}$ for type $A$ boundaries and $\alpha_{2}^{i}$ for type $D$ boundaries. 

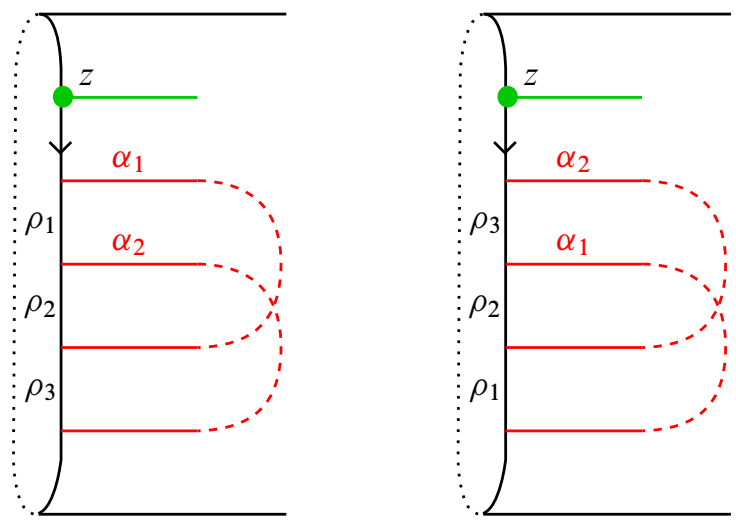

Figure 1: Boundary markings for type $A$ (left) and $D$ (right) boundaries on a bordered Heegaard diagram

We can associate a copy of the torus algebra to each boundary component, so that the Reeb chords on $\partial \Sigma$ correspond directly to the Reeb elements of the algebra. By abuse of notation, we often use $\rho_{I}^{i}$ to refer both to the Reeb chord on $\partial \Sigma$ and the corresponding algebra element in the corresponding copy of the torus algebra.

\section{D Type $D$ invariants}

Let $Y$ be a bordered 3-manifold with $k$ boundary components, and let $\mathcal{H}$ be an arced bordered Heegaard diagram representing $Y$ which is provincially admissible in the sense of [8, Definition 4.23]. Choose a complex structure $J$ on $\Sigma \times[0,1] \times \mathbb{R}$. To ensure transversality, the choice of $J$ must be generic; however, for the computations in this paper we may assume that $J$ splits as $J_{\Sigma} \times J_{D}$, where $J_{\Sigma}$ is a generic complex structure on $\Sigma$ and $J_{D}$ is a generic complex structure on $[0,1] \times \mathbb{R}$. Split complex structures provide enough flexibility for transversality when the projections to $\Sigma$ of all curves being considered are somewhere injective [7, Proposition 3.9]. Given these choices, we will associate to $\mathcal{H}$ a type $D$ multimodule $\widehat{\mathrm{CFD}}^{k}(\mathcal{H}, J)$ over $k$ copies of the torus algebra $\mathcal{A}\left(T^{2}\right)$. We will often suppress $J$ from the notation.

Let $\mathfrak{S}(\mathcal{H})$ be the set of unordered $g$-tuples $\boldsymbol{x}=\left(x_{1}, \ldots, x_{g}\right)$ which contain exactly one point on each $\beta$ curve, exactly one point on each $\alpha$ curve, and at most one point on each $\alpha$ arc. Elements of $\mathfrak{S}(\mathcal{H})$ fall into different $\operatorname{spin}^{c}$-structures according to how many $\alpha$ arcs are occupied on each boundary. $\widehat{\mathrm{CFD}}^{k}(\mathcal{H})$ is generated by $\mathfrak{S}(\mathcal{H})$ as a vector space over $\mathbb{F}_{2}$, and it splits as a direct sum over $\operatorname{spin}^{c}$-structures on $Y[8$, Lemma 4.21]. Each generator $\boldsymbol{x} \in \mathfrak{S}(\mathcal{H})$ comes equipped with an idempotent in the algebra associated to each boundary component; if $\boldsymbol{x}$ has exactly one $\alpha^{i}$ arc occupied, 
then the corresponding idempotent in $\mathcal{A}_{i}=\mathcal{A}\left(T^{2}\right)$ is $\iota_{1}^{i}$ if $\boldsymbol{x}$ contains a point on $\alpha_{1}^{i}$ and $\iota_{0}^{i}$ if $\boldsymbol{x}$ contains a point on $\alpha_{2}^{i}$.

The differential on $\widehat{\mathrm{CFD}}^{k}(\mathcal{H})$ counts $J$-holomorphic curves in $\Sigma \times[0,1] \times \mathbb{R}$ with appropriate boundary conditions (for precise statements of these conditions, see [8, Section 5.2]). These curves can be sorted into relative homology classes. For any $\boldsymbol{x}, \boldsymbol{y} \in \mathfrak{S}(\mathcal{H})$, let $\pi_{2}(\boldsymbol{x}, \boldsymbol{y})$ denote the set of homology classes of curves in $\Sigma \times[0,1] \times \mathbb{R}$ with boundary conditions consistent with a differential connecting $\boldsymbol{x}$ to $\boldsymbol{y}$. Computing the differential involves counting the holomorphic representatives for each homology class.

Under the projection $\Sigma \times[0,1] \times \mathbb{R} \rightarrow \Sigma$, a homology class $B \in \pi_{2}(\boldsymbol{x}, \boldsymbol{y})$ projects to an element of $H_{2}(\Sigma, \boldsymbol{\alpha} \cup \boldsymbol{\beta} \cup \partial \Sigma)$, called the domain of $B$. The class $B$ is determined by its domain. A domain is a linear combination of components of $\Sigma \backslash(\boldsymbol{\alpha} \cup \boldsymbol{\beta})$, which we call regions. Furthermore, the domain of any $B \in \pi_{2}(x, y)$ must satisfy the following conditions:

- The multiplicity of the region containing the basepoint $z$ is 0 .

- At each $p \in \boldsymbol{\alpha} \cap \boldsymbol{\beta}$, let $n_{1}(p), \ldots, n_{4}(p)$ be the multiplicities of the four regions with corners at $p$, counting counterclockwise starting from an $\alpha$. Then

$$
n_{1}(p)-n_{2}(p)+n_{3}(p)-n_{4}(p)= \begin{cases}1 & \text { if } p \in \boldsymbol{x} \backslash \boldsymbol{y} \\ -1 & \text { if } p \in \boldsymbol{y} \backslash \boldsymbol{x} \\ 0 & \text { otherwise }\end{cases}
$$

A domain is called positive if every region has nonnegative multiplicity. Only positive domains can support holomorphic representatives. Because the Heegaard diagram $\mathcal{H}$ is provincially admissible, there are a finite number of positive domains with multiplicity at most 1 in the regions adjacent to $\partial \Sigma$ (we will see that only these are relevant for computing $\widehat{\mathrm{CFD}}^{k}$ ). Finding them is a simple matter of linear algebra.

In addition to its domain $B$, a holomorphic curve that contributes to the differential of $\boldsymbol{x}$ also specifies a sequence of Reeb chords $\vec{\rho}=\left(\vec{\rho}^{1}, \ldots, \vec{\rho}^{k}\right)$, where $\vec{\rho}^{i}=\left(\rho_{I_{1}}^{i}, \ldots, \rho_{I_{n}}^{i}\right)$ are sequences corresponding to each boundary component of $\Sigma$. For each boundary component with one $\alpha^{i}$ arc occupied by $\boldsymbol{x}$, the pair $\left(B, \vec{\rho}^{i}\right)$ will satisfy the following conditions:

- the initial point (with respect to the boundary orientation) of $\rho_{I_{1}}^{i}$ lies on the same $\alpha^{i}$ arc as $\boldsymbol{x}$;

- for each $m>1$, the initial point of $\rho_{I_{m}}^{i}$ lies on the same $\alpha^{i}$ arc as the terminal point of $\rho_{I_{m-1}}^{i}$. 
A pair $\left(B, \vec{\rho}^{i}\right)$ satisfying the above conditions is called strongly boundary monotonic. For each boundary component with zero or two $\alpha^{i}$ arcs occupied by $\boldsymbol{x}$, we may assume that $\vec{\rho}^{i}=()$. The pair $(B, \vec{\rho})$ coming from a holomorphic curve will also satisfy the following property:

- the intersection of $B$ with the $i^{\text {th }}$ component of $\partial \Sigma$ is equal to the sum of the Reeb chords in $\vec{\rho}^{i}$ as elements of $H_{1}(\partial \Sigma, \boldsymbol{\alpha} \cap \partial \Sigma)$.

We say that the pair $(B, \vec{\rho})$ is compatible if it satisfies this condition and each $\left(B, \vec{\rho}^{i}\right)$ is strongly boundary monotonic (compare [8, Definition 5.68]).

Given generators $\boldsymbol{x}, \boldsymbol{y} \in \mathfrak{S}(\mathcal{H})$, a homology class $B \in \pi_{2}(\boldsymbol{x}, \boldsymbol{y})$, and a sequence of Reeb chords $\vec{\rho}$ such that $(B, \vec{\rho})$ is compatible, we can define $\mathcal{M}^{B}(\boldsymbol{x}, \boldsymbol{y}, \vec{\rho})$ to be the moduli space of $J$-holomorphic curves in $\Sigma \times[0,1] \times \mathbb{R}$ with domain $B$ and whose asymptotics specify the initial generator $\boldsymbol{x}$, the final generator $\boldsymbol{y}$, and the sequence of Reeb chords $\vec{\rho}$ (for the full definition, see [8, Section 5]).

The dimension of the moduli space $\mathcal{M}^{B}(\boldsymbol{x}, \boldsymbol{y}, \vec{\rho})$ is one less than the index $\operatorname{ind}(B, \vec{\rho})$, defined in [8, Definition 5.68]. In the special case of toroidal boundary, the index is given by

$$
\operatorname{ind}(B, \vec{\rho})=e(B)+n_{\boldsymbol{x}}(B)+n_{\boldsymbol{y}}(B)+\sum_{1 \leq i \leq k}\left[\frac{1}{2}\left|\vec{\rho}^{i}\right|+\sum_{j<l} L\left(\rho_{I_{j}}^{i}, \rho_{I_{l}}^{i}\right)\right],
$$

where

$$
e(B)=\chi(B)-\frac{1}{4} \# \text { acute corners }+\frac{1}{4} \# \text { obtuse corners }
$$

is the Euler measure of $B, n_{\boldsymbol{x}}(B)$ (respectively $n_{\boldsymbol{y}}(B)$ ) is the sum over $x_{i} \in \boldsymbol{x}$ (respectively $y_{i} \in \boldsymbol{y}$ ) of the average multiplicity in $B$ of the four regions incident to $x_{i}$ (respectively $\left.y_{i}\right),\left|\vec{\rho}^{i}\right|$ is the number of Reeb chords in the part of $\vec{\rho}$ associated to the $i^{\text {th }}$ component of $\partial \Sigma$, and $L\left(\rho_{I_{j}}, \rho_{I_{l}}\right)$ is a linking term for Reeb chords defined as follows:

$$
\begin{aligned}
\frac{1}{2} & =L\left(\rho_{1}, \rho_{2}\right)=L\left(\rho_{2}, \rho_{3}\right)=L\left(\rho_{12}, \rho_{3}\right)=L\left(\rho_{1}, \rho_{23}\right), \\
-\frac{1}{2} & =L\left(\rho_{2}, \rho_{1}\right)=L\left(\rho_{3}, \rho_{2}\right)=L\left(\rho_{3}, \rho_{12}\right)=L\left(\rho_{23}, \rho_{1}\right), \\
1 & =L\left(\rho_{12}, \rho_{23}\right), \\
-1 & =L\left(\rho_{23}, \rho_{12}\right), \\
0 & =L\left(\rho_{I_{j}}, \rho_{I_{l}}\right) \quad \text { for all other pairs of } I_{j} \text { and } I_{l} .
\end{aligned}
$$

The differential counts $J$-holomorphic curves in moduli spaces with dimension 0 , so we only need to consider domains and Reeb chords with $\operatorname{ind}(B, \vec{\rho})=1$.

To define the differential we need one more piece of notation. If $\rho_{I}^{i}$ represents a Reeb chord on the $i^{\text {th }}$ boundary component of $\Sigma$, let $a\left(\rho_{I}^{i}\right)$ denote the corresponding 
element of $\mathcal{A}_{i}$, the copy of the torus algebra associated to the $i^{\text {th }}$ boundary component. If $\vec{\rho}^{i}=\left(\rho_{I_{1}}^{i}, \ldots, \rho_{I_{n}}^{i}\right)$ is a sequence of Reeb chords on the $i^{\text {th }}$ boundary, let $a\left(\vec{\rho}^{i}\right)$ denote the element $\rho_{I_{1}}^{i} \rho_{I_{2}}^{i} \cdots \rho_{I_{n}}^{i} \in \mathcal{A}_{i}$, and if $\vec{\rho}=\left(\vec{\rho}^{1}, \ldots, \vec{\rho}^{k}\right)$, let $a(\vec{\rho})$ denote the element $a\left(\vec{\rho}^{1}\right) \otimes \cdots \otimes a\left(\vec{\rho}^{k}\right) \in \mathcal{A}_{1} \otimes \cdots \otimes \mathcal{A}_{k}$. We now define the differential on $\widehat{\mathrm{CFD}}^{k}$ as follows: For any $\boldsymbol{x} \in \mathfrak{S}(\mathcal{H})$,

$$
\partial(\boldsymbol{x})=\sum_{\boldsymbol{y} \in \mathfrak{S}(\mathcal{H})} \sum_{B \in \pi_{2}(\boldsymbol{x}, \boldsymbol{y})} \sum_{\{\vec{\rho} \mid \operatorname{ind}(B, \vec{\rho})=1,}\left(\# \mathcal{M}^{B}(\boldsymbol{x}, \boldsymbol{y}, \vec{\rho})\right) a(\vec{\rho}) \otimes \boldsymbol{y},
$$

$(B, \vec{\rho})$ is compatible $\}$

where the count of a moduli space is taken mod 2 .

Given a domain $B$ and a sequence of Reeb chords $\vec{\rho}$, we will say that the pair $(B, \vec{\rho})$ contributes to the differential from $\boldsymbol{x}$ to $\boldsymbol{y}$ if the mod 2 count of the relevant moduli space is nonzero; note, however, that the contribution may be zero if $a(\vec{\rho}) \otimes \boldsymbol{y}$ is zero. Thus a pair may either contribute a nonzero term to the differential, contribute zero to the differential, or not contribute to the differential (while the effect on the differential is the same in the last two cases, we find it helpful to distinguish them). Similarly, we will say that a sequence of Reeb chords $\vec{\rho}$ contributes $a(\vec{\rho}) \otimes \boldsymbol{y}$ to the differential from $\boldsymbol{x}$ to $\boldsymbol{y}$ if the sum of the counts of the relevant moduli space for all compatible $B$ in $\pi_{2}(\boldsymbol{x}, \boldsymbol{y})$ is nonzero.

Note that the multimodule $\widehat{\mathrm{CFD}}^{k}(\mathcal{H}, J)$ depends on the choices of $\mathcal{H}$ and $J$. However, its quasi-isomorphism type is an invariant of the bordered manifold $Y$. We denote this quasi-isomorphism class by $\widehat{\mathrm{CFD}}^{k}(Y)$. We will deviate slightly from the notation introduced here when $k \leq 2$ in order to agree with existing notation. That is, we will omit the superscript in $\widehat{\mathrm{CFD}}^{1}$, and we will write $\widehat{\mathrm{CFDD}}$ instead of $\widehat{\mathrm{CFD}}^{2}$.

\section{E Type $A$ invariants}

Let $Y$ be a bordered 3-manifold with $k$ boundary components and let $\mathcal{H}$ be a provincially admissible arced bordered Heegaard diagram representing $Y$ and $J$ a chosen complex structure, as before. We can define a type $A$ multimodule over $k$ copies of the torus algebra, denoted $\widehat{\mathrm{CFA}}^{k}(\mathcal{H})$. In this paper, we will never need to compute $\widehat{\mathrm{CFA}}^{k}(\mathcal{H})$. However, as a computational trick we will make use of the relationship between $\widehat{\mathrm{CFA}}^{k}(\mathcal{H})$ and $\widehat{\mathrm{CFD}}^{k}(\mathcal{H})$, so it will be helpful to state the definition.

$\widehat{\mathrm{CFA}}^{k}(\mathcal{H})$ is generated by the same set $\mathfrak{S}(\mathcal{H})$ that generates $\widehat{\mathrm{CFD}}^{k}(\mathcal{H})$. In the torus boundary case, the definition of the differential and higher multiplications involves the same moduli spaces of $J$-holomorphic curves that are considered for $\widehat{\mathrm{CFD}}^{k}(\mathcal{H})$. We will assume for the sake of comparison that the Reeb chords on the boundary are labeled the same as if we were computing $\widehat{\mathrm{CFD}}^{k}(\mathcal{H})$, so that for a given domain 
the compatible sequences of Reeb chords $\vec{\rho}$ and the moduli spaces $\mathcal{M}^{B}(\boldsymbol{x}, \boldsymbol{y}, \vec{\rho})$ are exactly the same. However, with this convention we must change the algebra elements in the $\mathcal{A}_{\infty}$ operation, since normally the Reeb chords are labeled in the opposite order for type $A$ Heegaard diagrams. Let the function $\bar{a}$ be the same as $a$ except that it also interchanges $\rho_{1}^{i}$ with $\rho_{3}^{i}$ and $\rho_{12}^{i}$ with $\rho_{23}^{i}$. Then given a generator $\boldsymbol{x} \in \mathfrak{S}(\mathcal{H})$ and sequence of Reeb chords $\vec{\rho}=\left(\vec{\rho}^{1}, \ldots, \vec{\rho}^{k}\right)$,

$$
m\left(\boldsymbol{x}, \bar{a}\left(\vec{\rho}^{1}\right), \ldots, \bar{a}\left(\vec{\rho}^{k}\right)\right)=\sum_{\boldsymbol{y} \in \mathfrak{S}(\mathcal{H})} \sum_{\substack{\left\{B \in \pi_{2}(\boldsymbol{x}, \boldsymbol{y}) \mid \\ \text { ind }(B, \vec{\rho})=1,(B, \vec{\rho}) \text { compatible }\right\}}}\left(\# \mathcal{M}^{B}(\boldsymbol{x}, \boldsymbol{y}, \vec{\rho})\right) \boldsymbol{y},
$$

where we think of $\bar{a}\left(\vec{\rho}^{i}\right)$ as an element of $\mathcal{A}_{i}^{\otimes\left|\vec{\rho}^{i}\right|}$, and where the moduli space counts are taken mod 2 .

\section{F Tensor products and the pairing theorem}

For a bordered manifold with many boundary components, we can define bordered invariants which are type $D$ with respect to some boundaries and type $A$ with respect to others. These invariants can be obtained from $\widehat{\mathrm{CFD}}^{k}$ by taking the box tensor product with the bimodule $\widehat{\mathrm{CFAA}}(\mathbb{I})$, which can be found in [10, Figure 21]. An alternative shorthand algorithm for converting to type $D$ boundaries to type $A$ is described in [4, Section 2.3].

Bordered invariants satisfy a pairing theorem [10, Theorem 11]. Given a bordered invariant for $Y_{1}$ which is type $A$ with respect to the $i^{\text {th }}$ boundary component and a bordered invariant for $Y_{2}$ which is type $D$ with respect to the $j^{\text {th }}$ boundary component, we can compute the box tensor product of the two multimodules with respect to the corresponding copies of the torus algebra, assuming the modules are appropriately bounded. The pairing theorem states that up to $\mathcal{A}_{\infty}$-homotopy equivalence, the result is the bordered invariant for the manifold obtained by gluing the $i^{\text {th }}$ boundary of $Y_{1}$ to the $j^{\text {th }}$ boundary of $Y_{2}$.

In this paper, we will work primarily with type $D$ modules, and convert only one boundary component at a time to type $A$ in order to tensor with another type $D$ module.

\section{G Useful results for computation}

This section collects a handful of results that are useful when explicitly computing a type $D$ bordered invariant.

The first is a slight rephrasing of [6, Proposition 2.1]: 
Proposition 2.3 (a) For a given boundary component, the only nonempty sequences of Reeb chords which can contribute nonzero terms to the differential in $\widehat{\mathrm{CFD}}^{k}$ are $\left(\rho_{1}^{i}\right),\left(\rho_{2}^{i}\right),\left(\rho_{3}^{i}\right),\left(\rho_{1}^{i}, \rho_{2}^{i}\right),\left(\rho_{2}^{i}, \rho_{3}^{i}\right),\left(\rho_{1}^{i}, \rho_{2}^{i}, \rho_{3}^{i}\right)$ and $\left(\rho_{123}^{i}\right)$.

(b) Furthermore, if $B \in \pi_{2}(\boldsymbol{x}, \boldsymbol{y})$ contributes with $\vec{\rho}^{i}=\left(\rho_{2}^{i}\right)$ or $\vec{\rho}^{i}=\left(\rho_{1}^{i}, \rho_{2}^{i}\right)$, then $\boldsymbol{y}$ contains a point on $\alpha_{2}^{i}$. If $B$ contributes and $\vec{\rho}^{i}$ is $\left(\rho_{1}^{i}\right),\left(\rho_{3}^{i}\right),\left(\rho_{123}^{i}\right)$ or $\left(\rho_{2}^{i}, \rho_{3}^{i}\right)$, then $\boldsymbol{y}$ contains a point on $\alpha_{1}^{i}$.

In particular, this proposition implies that only domains with multiplicity 0 or 1 in every region that intersects $\partial \Sigma$ can contribute nontrivially to the differential in $\widehat{\mathrm{CFD}}^{k}$. For provincially admissible Heegaard diagrams this ensures that there is a finite number of positive domains to consider.

Proposition 2.3 also implies that (5) can be simplified for type $D$ computations.

Lemma 2.4 If the pair $(B, \vec{\rho})$ contributes a nonzero term to the differential of $\widehat{\mathrm{CFD}}^{k}$, then the index of the pair is given by

(6) $\operatorname{ind}(B, \vec{\rho})=\operatorname{ind}(B)=e(B)+n_{\boldsymbol{x}}(B)+n_{\boldsymbol{y}}(B)+\frac{1}{2} \#\left\{Z \in \pi_{0}(\partial \Sigma) \mid Z \cap B \neq \varnothing\right\}$.

In particular, the index depends only on $B$.

Proof We examine the term in brackets in (5). For the $i^{\text {th }}$ component of $\partial \Sigma$, there is a contribution to the index of

$$
\frac{1}{2}\left|\vec{\rho}^{i}\right|+\sum_{j<l} L\left(\rho_{I_{j}}^{i}, \rho_{I_{l}}^{i}\right)
$$

We can evaluate this term for each of the sequences of Reeb chords allowed by Proposition 2.3. If $\vec{\rho}^{i}$ is $\left(\rho_{1}^{i}\right),\left(\rho_{2}^{i}\right),\left(\rho_{3}^{i}\right)$ or $\left(\rho_{123}^{i}\right)$, then $\left|\vec{\rho}^{i}\right|=1$ and there are no linking terms. If $\vec{\rho}^{i}$ is $\left(\rho_{1}^{i}, \rho_{2}^{i}\right)$ or $\left(\rho_{2}^{i}, \rho_{3}^{i}\right)$, then $\left|\vec{\rho}^{i}\right|=2$, and there is one linking term, with a value of $-\frac{1}{2}$. If $\vec{\rho}^{i}$ is $\left(\rho_{1}^{i}, \rho_{2}^{i}, \rho_{3}^{i}\right)$, then $\left|\vec{\rho}^{i}\right|=3$, and the two nonzero linking terms $L\left(\rho_{1}^{i}, \rho_{2}^{i}\right)$ and $L\left(\rho_{2}^{i}, \rho_{3}^{i}\right)$ evaluate to $-\frac{1}{2}$. In any of these cases, the total contribution to the index is $\frac{1}{2}$. The only other possibility is that $\vec{\rho}^{i}=()$, which happens when $B$ does not contain any regions adjacent to the $i^{\text {th }}$ boundary component of $\Sigma$. In this case, the contribution of $\vec{\rho}^{i}$ to the index is 0 . Summing over all boundary components yields

$$
\sum_{1 \leq i \leq k}\left[\frac{1}{2}\left|\vec{\rho}^{i}\right|+\sum_{j<l} L\left(\rho_{I_{j}}^{i}, \rho_{I_{l}}^{i}\right)\right]=\frac{1}{2} \#\left\{Z \in \pi_{0}(\partial \Sigma) \mid Z \cap B \neq \varnothing\right\} .
$$

Lemma 2.4 allows us to exclude a domain $B$ from consideration in computing $\widehat{\mathrm{CFD}}^{k}$ if $\operatorname{ind}(B) \neq 1$, without needing to consider all sequences of Reeb chords compatible with $B$. 
In practice, computing $\widehat{\mathrm{CFD}}^{k}$ from a Heegaard diagram begins by writing down all positive domains $B \in \pi_{2}(\boldsymbol{x}, \boldsymbol{y})$ for each pair of generators $\boldsymbol{x}$ and $\boldsymbol{y}$, and then eliminating as many domains as possible using Proposition 2.3 and Lemma 2.4. At some point, however, it is necessary to prove that a given domain/Reeb chord pair does contribute to the differential. The following proposition asserts that a domain which can be realized as an immersed polygon always contributes.

Proposition 2.5 Let $P$ be a $2 n$-gon with edges numbered consecutively, and suppose that there is map $P \stackrel{u}{\rightarrow} \Sigma$ satisfying the following conditions:

- $\left.u\right|_{\partial P}$ takes even edges of $P$ to $\boldsymbol{\beta}$, odd edges of $P$ to $\boldsymbol{\alpha} \cup \partial \Sigma$, and corners to acute corners;

- $u$ is an immersion, except at the preimages of $\boldsymbol{\alpha} \cap \partial \Sigma$;

- for each boundary component of $\Sigma$, at most one edge of $P$ maps to $\alpha_{1}^{i} \cup \alpha_{2}^{i}$, and for each $\beta \in \boldsymbol{\beta}$, at most one edge of $P$ maps to $\beta$.

The image of $u$ covers each region in $\Sigma$ with a certain multiplicity; let $B(u)$ be the corresponding positive domain. The image of $\partial P$ determines a sequence of Reeb chords $\vec{\rho}(u)$, with the chords in the image of a single edge ordered according to the boundary orientation on $\partial P$. If $B(u) \in \pi_{2}(\boldsymbol{x}, \boldsymbol{y})$ for some generators $\boldsymbol{x}$ and $\boldsymbol{y}$ in the middle spin ${ }^{c}$-structure, then $(B(u), \vec{\rho}(u))$ is compatible, and $\# \mathcal{M}^{B}(\boldsymbol{x}, \boldsymbol{y}, \vec{\rho}) \equiv$ $1(\bmod 2)$.

Proof A holomorphic curve in $\Sigma \times[0,1] \times \mathbb{R}$ is equivalent to a holomorphic map of a Riemann surface with boundary into $\Sigma$ along with a branched covering map of that surface over the unit disk $D^{2} \subset \mathbb{C}$; see [27, Lemma 3.6]. For a specific domain, we look at Riemann surfaces which map onto the given domain in $\Sigma$, such that the preimages of the $\alpha$ arcs (together with boundary Reeb chords) and $\beta$ arcs map to the right and left boundaries, respectively, in the projection to $D^{2}$, and the preimages of the $\boldsymbol{x}$ and $\boldsymbol{y}$ corners map to $-i$ and $i$, respectively.

In this case, we already have a map from the polygon $P$ to $\Sigma$. There is a unique choice of complex structure on $P$ that makes $u$ holomorphic (induced by pulling back the complex structure on $\Sigma$ ). So we need to show that with this fixed complex structure, there is a unique $n$-fold branched covering map to $D^{2}$ up to an $\mathbb{R}$ action.

First choose a biholomorphic map from $P$ to the upper half plane $\mathbb{H}$, which takes one of the $\boldsymbol{y}$ corners to $\infty$, and the other corners to points $x_{1}, x_{2}, \ldots, x_{2 n-1}$ along the real axis. We now want to find a degree $n$ map $\mathbb{H} \rightarrow \mathbb{H}$ which takes $x_{i}$ to 0 for $i$ odd and to $\infty$ for $i$ even, and takes $\infty$ to $\infty$. Such a map is given by

$$
z \mapsto \frac{\left(z-x_{1}\right)\left(z-x_{3}\right) \cdots\left(z-x_{2 n-1}\right)}{\left(z-x_{2}\right)\left(z-x_{4}\right) \cdots\left(z-x_{2 n-2}\right)} .
$$




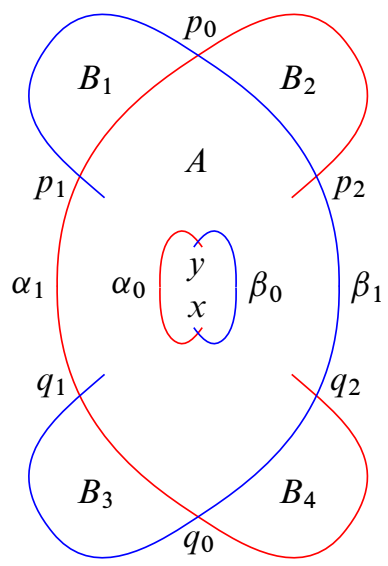

Figure 2: The annuli in Proposition 2.6 have the form of the annulus $A$ above combined with one of the bigons $B_{i}$, where the $\alpha$ arcs in the boundary of the annulus may contain segments of $\partial \Sigma$.

This map is unique up to scaling. Finally we can find a biholomorphic map from $\mathbb{H}$ to $D^{2}$ which takes 0 to $-i$ and $\infty$ to $+i$. Composing these three maps gives the desired $k$-fold branched cover $P \rightarrow D^{2}$.

Another common situation in which the moduli space of holomorphic curves can be understood is pictured in Figure 2. The following appears in [6], but we recall the proof here in order to introduce notation and reasoning that will be useful later.

Proposition 2.6 Suppose a Heegaard diagram contains (an immersed copy of) an annulus $A$, as in Figure 2, and one or more of the bigons $B_{1}, \ldots, B_{4}$, where $\alpha$ arcs may contain segments of $\partial \Sigma$, and where the ends of $\alpha_{1}$ leave $A$ by crossing $\beta_{0}$ and the ends of $\beta_{1}$ leave $A$ by crossing $\alpha_{0}$. Let $D_{i}$ denote the domain corresponding to the union of $A$ and $B_{i}$. Then either $D_{1}$ and $D_{3}$ count toward the differential and $D_{2}$ and $D_{4}$ do not, or vice versa, depending on the choice of complex structure $J$ on $\Sigma$.

Proof Let $A_{r}$ denote the standard annulus, $S^{1} \times[0, r]$ with a fixed complex structure. For a unique positive number $r$ there is a holomorphic map $u: A_{r} \rightarrow A$ taking $S^{1} \times\{0\}$ to the inner boundary of $D$, namely $D \cap\left(\alpha_{0} \cup \beta_{0}\right)$, and taking $S^{1} \times\{r\}$ to the outer boundary $D \cap\left(\alpha_{1} \cup \beta_{1}\right)$. This map is unique up to rotation in the $S^{1}$ factor. Let $a_{0}$ and $b_{0}$ denote the inverse images in $S^{1} \times\{0\}$ of $\alpha_{0}$ and $\beta_{0}$, respectively. Let $a_{1}$ and $b_{1}$ denote the respective inverse images of $\alpha_{1}$ and $\beta_{1}$ in $S^{1} \times\{r\}$. Define $\Theta_{A}^{x, y}$ to be $l\left(a_{0}\right) / l\left(b_{0}\right)$, the ratio of the lengths of the preimages of the $\alpha$ and $\beta$ arcs on the boundary of $A$ which contains $x$ and $y$. Similarly, define $\Theta_{A}^{p_{0}, q_{0}}$ to be 
$l\left(a_{1}\right) / l\left(b_{1}\right)$. The domain $A$ will have a holomorphic representative if $\Theta_{A}^{x, y}=\Theta_{A}^{p_{0}, q_{0}}$ [27, Lemma 9.3], but for a generic choice of complex structure this will not be the case.

Now consider the domain $D_{1}$. It is an annulus with one obtuse corner at $p_{1}$. There is a one parameter family of conformal structures depending on how much we cut into the annulus along the $\alpha$ or $\beta$ arc at the obtuse corner. We specify the length of this cut by a parameter $c$, where $c<0$ corresponds to cutting along $\alpha_{1}$ and $c>0$ corresponds to cutting along $\beta_{1}$. The cut approaches $p_{0}$ as $c \rightarrow-\infty$ and it approaches $\alpha_{0}$ as $c \rightarrow \infty$. For any value of $c$ there is a holomorphic map $U^{c}: A_{r} \rightarrow D_{1}$, unique up to rotation in the $S^{1}$ factor of $A_{r}$. We can define $\theta_{D_{1}}^{x, y}(c)$ and $\theta_{D_{1}}^{p_{1}}, q_{0}(c)$ analogously to $\Theta_{A}^{x, y}$ and $\Theta_{A}^{p_{0}}, q_{0}$, as the ratio of the lengths of the $\alpha$ and $\beta$ components on the corresponding boundary of $D_{1}$.

As the cutting parameter varies, $D_{1}$ will have a holomorphic representative each time $\theta_{D_{1}}^{x, y}(c)=\theta_{D_{1}}^{p_{1}, q_{0}}(c)$, and thus the number of holomorphic representatives is determined by the number of zeros of $\theta_{D_{1}}^{x, y}-\theta_{D_{1}}^{p_{1}, q_{0}}$. The end behavior of $\theta_{D_{1}}^{x, y}-\theta_{D_{1}}^{p_{1}, q_{0}}$ determines the mod 2 count of these zeros. As $c$ approaches $\infty$, the cut along $\beta_{1}$ from $p_{1}$ approaches $\alpha_{0}$. In this limit $\theta_{D_{1}}^{x, y}$ becomes very large and $\theta_{D_{1}}^{p_{1}, q_{0}}$ becomes very small, so $\theta_{D_{1}}^{x, y}-\theta_{D_{1}}^{p_{1}, q_{0}} \rightarrow+\infty$. In the other extreme, we cut along $\alpha_{1}$ from $p_{1}$ to $p_{0}$. The limit is a broken flow where the bigon $B_{1}$ is pinched off from the annulus $A$. In this limit $\theta_{D_{1}}^{x, y}=\Theta_{A}^{x, y}$ and $\theta_{D_{1}}^{p_{1}, q_{0}}=\Theta_{A}^{p_{0}, q_{0}}$. Therefore, the domain $D_{1}$ will contribute to the differential in $\widehat{\mathrm{CFD}}^{k}$ if and only if $\Theta_{A}^{p_{0}, q_{0}}>\Theta_{A}^{x, y}$.

The domains $D_{2}, D_{3}$ and $D_{4}$ can be analyzed in the same way. For $D_{3}$ the results are the same: cutting along $\beta_{1}$ from $q_{1}$ to $\alpha_{0}$ makes $\theta_{D_{3}}^{x, y}-\theta_{D_{3}}^{p_{0}, q_{1}}$ approach $+\infty$, and cutting along $\alpha_{1}$ from $q_{1}$ to $q_{0}$ yields $\Theta_{D_{3}}^{x, y}-\theta_{D_{3}}^{p_{0}, q_{1}^{3}}=\Theta_{A}^{x, y}-\Theta_{A}^{p_{0}, q_{0}}$, so $D_{3}$ contributes if and only if $\Theta_{A}^{p_{0}, q_{0}}>\Theta_{A}^{x, y}$. The domains $D_{2}$ and $D_{4}$, on the other hand, contribute if and only if $\Theta_{A}^{x, y}>\Theta_{A}^{p_{0}, q_{0}}$.

Note that since annulus in Figure 2 is only required to be immersed, some points or segments of arcs may be identified. For instance, it may be that $x$ and $y$ map to the same point on the Heegaard diagram; see Figure 3.

We will often encounter annular domains that fit the form of the annuli in Proposition 2.6 except that one boundary component has more than one $\alpha$ segment and more than one $\beta$ segment. For instance, the bigon $B_{1}$ might be replaced with a quadrilateral. In practice, quadrilaterals behave like bigons in this context, but it is not immediately apparent how to extend the proof of Proposition 2.6 for more general annuli. Instead, we will use the following proposition to simplify a domain by pinching off an extra $\alpha$ or $\beta$ arc. 

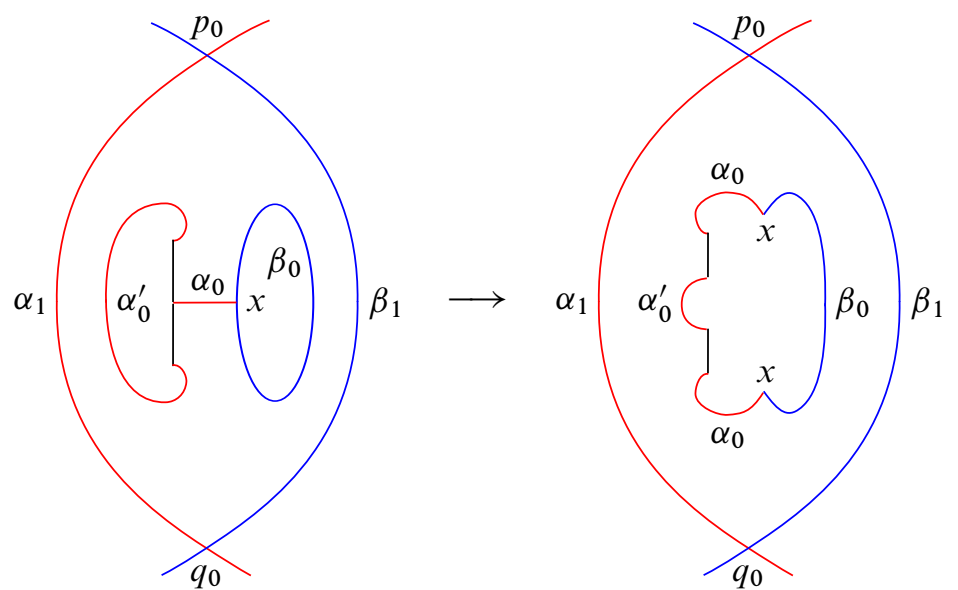

Figure 3: The partial Heegaard diagram pictured on the left contains an annulus $A$ in the sense of Proposition 2.6. The straight black lines represent segments of $\partial \Sigma$. The annulus is immersed (except at the preimages of $\alpha \cap \partial \Sigma$ ), but not embedded; in particular, $x$ and $y$ in Figure 2 map to the same point, $x$. To realize this domain in the form of Figure 2, cut along the arc $\alpha_{0}$ from $x$ to $\partial \Sigma$, producing the picture on the right.

Proposition 2.7 Let $\gamma$ be an arc in a domain $D$ which is a small push off of one of the $\beta$ segments or one of the $\alpha$ segments (possibly containing Reeb chords) in $\partial D$, as pictured here:
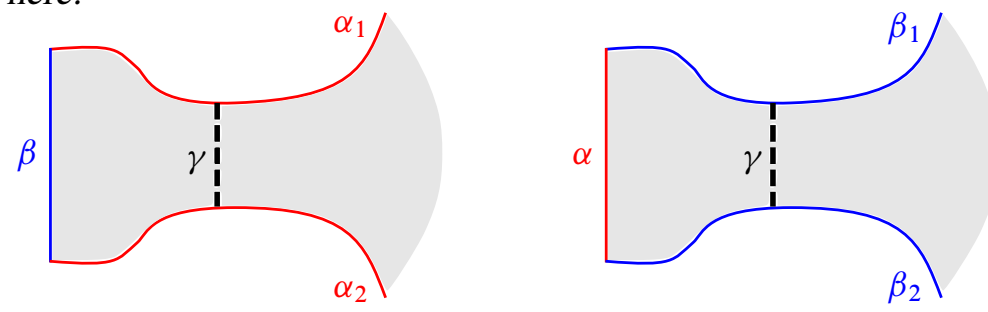

Assume that $\gamma$ only passes through regions with multiplicity 1 in $D$. Let $D^{\prime}$ be the domain which results from collapsing $\gamma$ to a point and removing the bigon on the left. Let $\vec{\rho}$ be a sequence of Reeb chords compatible with $D$, and let $\vec{\rho}^{\prime}$ be obtained from $\vec{\rho}$ by removing any Reeb chords on the bigon on the left. Then for an appropriate choice of complex structure, namely one in which the arc $\gamma$ is sufficiently short, $(D, \vec{\rho})$ contributes to $\widehat{\mathrm{CFD}}^{k}$ if and only if $\left(D^{\prime}, \vec{\rho}^{\prime}\right)$ would contribute.

Proof Fix a neighborhood $U_{\gamma}$ of $\gamma$ in $D$. Given any complex structure $J$ on $D$, there is some (unique) $\epsilon>0$ such that $U_{\gamma}$ maps holomorphically to $[0,1] \times(-\epsilon, \epsilon)$ with $\left.U_{\gamma}\right|_{\partial D}$ mapping to $\{0,1\} \times(-\epsilon, \epsilon)$. On $U_{\gamma}$, we have that $J$ is equivalent to the pullback of the standard complex structure on this rectangle. 
Consider the one parameter family of complex structures $J_{t}$, for $t>0$, such that $J_{t}$ agrees with $J$ outside of $U_{\gamma}$, and $J_{t}$ is scaled on $U_{\gamma}$ so that $U_{\gamma}$ is identified with the rectangle $[0,1] \times(-t, t)$. As $t$ goes to infinity, $U_{\gamma}$ is pinched in the direction of $\gamma$ and stretched in the direction transverse to $\gamma$ - effectively the arc $\gamma$ becomes shorter. The limiting complex structure $J_{\infty}$ corresponds to $\gamma$ being pinched to a single point, resulting in a bigon $B$ and the domain $D^{\prime}$ joined at a point. A $J_{\infty}$-holomorphic curve with domain $D$ splits as a holomorphic map to the bigon and a holomorphic curve with domain $D^{\prime}$. By the Riemann mapping theorem, there is an $\mathbb{R}$ family of holomorphic maps from the standard bigon to $B$, and precisely one once the point of contact with the preimage of $D^{\prime}$ is determined. Therefore the existence of $J_{\infty}$-holomorphic curves with domain $D$ is equivalent to the existence of $J_{\infty}$-holomorphic curves with domain $D^{\prime}$. $J_{\infty}$ is not a valid complex structure to choose when computing $\widehat{\mathrm{CFD}}^{k}$, but we can choose $J_{t}$ for arbitrarily large $t$ and standard compactness and gluing arguments imply that for $t$ sufficiently large, $D$ has a $J_{t}$-holomorphic representative if and only if it has a $J_{\infty}$-holomorphic representative (this follows from the same argument used in the proof of [8, Proposition 9.6], making use of [1, Theorem 10.3] and [7, Propsition A.2]). Thus for a complex structure with the arc $\gamma$ sufficiently pinched, the statement of the proposition holds.

Finally, we discuss how $\mathcal{A}_{\infty}$ relations can be used to deduce which domains count toward the differential, even if we are computing $\widehat{\mathrm{CFD}}^{k}$. The key is the following observation, which was implicitly used in the proof of [10, Proposition 10.5] to determine the contribution of $\rho_{123}$.

Lemma 2.8 A sequence of Reeb chords $\vec{\rho}=\left(\vec{\rho}^{1}, \ldots, \vec{\rho}^{k}\right)$ contributes $a(\vec{\rho}) \otimes \boldsymbol{y}$ to the differential of $\boldsymbol{x}$ in $\widehat{\mathrm{CFD}}^{k}$ if and only if $m\left(\boldsymbol{x}, \bar{a}\left(\vec{\rho}^{1}\right), \ldots, \bar{a}\left(\vec{\rho}^{k}\right)\right)=\boldsymbol{y}$ in $\widehat{\mathrm{CFA}}^{k}$.

Proof This follows directly from the definitions of $\widehat{\mathrm{CFD}}^{k}$ and $\widehat{\mathrm{CFA}}^{k}$, since both involve counts of the same moduli spaces. For a given domain $B \in \pi_{2}(\boldsymbol{x}, \boldsymbol{y})$ that is compatible with $\vec{\rho}$, the pair $(B, \vec{\rho})$ may contribute $a(\vec{\rho}) \otimes \boldsymbol{y}$ to $\partial \boldsymbol{x}$ in $\widehat{\mathrm{CFD}^{k}}$, and it may contribute the operation $m\left(\boldsymbol{x}, \bar{a}\left(\vec{\rho}^{1}\right), \ldots, \bar{a}\left(\vec{\rho}^{k}\right)\right)=\boldsymbol{y}$ to $\widehat{\mathrm{CFA}}^{k}$. In both cases, the pair contributes if and only if $\#\left(\mathcal{M}^{B}(\boldsymbol{x}, \boldsymbol{y}, \vec{\rho})\right) \equiv 1(\bmod 2)$. Summing over all domains $B$ compatible with $\vec{\rho}$ gives the total contribution of $\vec{\rho}$ and completes the proof.

Recall that we say $\vec{\rho}$ contributes $a(\vec{\rho}) \otimes \boldsymbol{y}$ to the differential of $\widehat{\mathrm{CFD}}^{k}$ if the relevant counts of moduli spaces are nonzero, even if the contribution is zero. It is possible that $\vec{\rho}$ contributes nontrivially to $\widehat{\mathrm{CFA}}^{k}$ while the contribution to $\widehat{\mathrm{CFD}}^{k}$ is zero.

Lemma 2.8 is most useful for checking if domains contribute to $\widehat{\mathrm{CFD}}^{k}$ when $\vec{\rho}$ contains the long chord $\rho_{123}^{i}$ for some boundary component. For example, suppose in the one 
boundary case that $\vec{\rho}=\left(\rho_{123}\right)$ is compatible with a domain $B$ from $\boldsymbol{x}$ to $\boldsymbol{y}$. If the domain is too complicated to understand the moduli space $\mathcal{M}^{B}(\boldsymbol{x}, \boldsymbol{y}, \vec{\rho})$ directly, we can instead ask whether $(B, \vec{\rho})$ contributes the operation $m\left(\boldsymbol{x}, \rho_{123}\right)=\boldsymbol{y}$ to $\widehat{\mathrm{CFA}}$. To answer this, we consider the $\mathcal{A}_{\infty}$ relation (3) corresponding to $\boldsymbol{x}$ and $\vec{\rho}^{\prime}=\left(\rho_{12}, \rho_{3}\right)$. The relation says that

$$
0=m\left(\boldsymbol{x}, \mu\left(\rho_{12}, \rho_{3}\right)\right)+m\left(m\left(\boldsymbol{x}, \rho_{12}\right), \rho_{3}\right) .
$$

The first term is just $m\left(\boldsymbol{x}, \rho_{123}\right)$, the operation we are interested in, and the second term might be easier to analyze. If $m\left(\boldsymbol{x}, \rho_{12}\right)=0$, for instance, then the second term in the relation is 0 , and thus $(B, \vec{\rho})$ does not contribute to $\widehat{\mathrm{CFA}}$ or to $\widehat{\mathrm{CFD}}$.

\section{H Gradings}

Bordered Heegaard Floer invariants can be equipped with a relative grading on each $\operatorname{spin}^{c}-$ structure as described in [8, Chapter 10] and [10, Section 6.5]. We recall here the construction of these gradings for manifolds with only torus boundary components. We will only discuss the refined grading.

Let $Y$ be a bordered manifold represented by a bordered Heegaard diagram $\mathcal{H}$. Let $\widehat{\mathrm{CF}}(\mathcal{H})$ denote the relevant bordered Heegaard Floer invariant. The gradings for $\widehat{\mathrm{CF}}(\mathcal{H})$ lie in a noncommutative group which depends on the number and type of boundary components. We will denote this group $G_{n, m}$ where $n$ is the number of type $D$ boundary components of $Y$ and $m$ is the number of type $A$ boundary components. $G_{n, m}$ is generated by tuples $\left(j ; a_{1}, b_{1} ; a_{2}, b_{2} ; \ldots ; a_{n+m}, b_{n+m}\right)$, where every entry is in $\frac{1}{2} \mathbb{Z}$, and $a_{i}+b_{i} \in \mathbb{Z}$ for each $i$. We refer to $j$ as the Maslov component of the grading. Multiplication on this group is defined as follows:

$$
\begin{aligned}
& \left(j ; a_{1}, b_{1} ; a_{2}, b_{2} ; \ldots ; a_{n+m}, b_{n+m}\right) \cdot\left(j^{\prime} ; a_{1}^{\prime}, b_{1}^{\prime} ; a_{2}^{\prime}, b_{2}^{\prime} ; \ldots ; a_{n+m}^{\prime}, b_{n+m}^{\prime}\right)= \\
& \quad\left(j+j^{\prime}+C ; a_{1}+a_{1}^{\prime}, b_{1}+b_{1}^{\prime} ; a_{2}+a_{2}^{\prime}, b_{2}+b_{2}^{\prime} ; \ldots ; a_{n+m}+a_{n+m}^{\prime}, b_{n+m}+b_{n+m}^{\prime}\right),
\end{aligned}
$$

where the correction term $C$ is given by

$$
C=\left|\begin{array}{ll}
a_{1} & a_{1}^{\prime} \\
b_{1} & b_{1}^{\prime}
\end{array}\right|+\cdots+\left|\begin{array}{ll}
a_{n} & a_{n}^{\prime} \\
b_{n} & b_{n}^{\prime}
\end{array}\right|+\left|\begin{array}{ll}
a_{n+1}^{\prime} & a_{n+1} \\
b_{n+1}^{\prime} & b_{n+1}
\end{array}\right|+\cdots+\left|\begin{array}{ll}
a_{n+m}^{\prime} & a_{n+m} \\
b_{n+m}^{\prime} & b_{n+m}
\end{array}\right| .
$$

Given generators $\boldsymbol{x}$ and $\boldsymbol{y}$, a domain in $B \in \pi_{2}(\boldsymbol{x}, \boldsymbol{y})$ can be given a grading in $G_{n, m}$ [8, Definition 10.1]. The Maslov component of $\operatorname{gr}(B)$ is given by

$$
-e(B)-n_{\boldsymbol{x}}(B)-n_{\boldsymbol{y}}(B),
$$

where $e(B), n_{\boldsymbol{x}}(B)$ and $n_{\boldsymbol{y}}(B)$ are the same quantities that appear in the index formula (5). For each $1 \leq i \leq n+m$, let $\gamma_{i}$ denote the intersection of $\partial B$ with the $i^{\text {th }}$ 
boundary component of $\mathcal{H}$, which can be thought of as a linear combination of the Reeb chords $\rho_{1}^{i}, \rho_{2}^{i}$ and $\rho_{3}^{i}$. If $\gamma_{i}=c_{1} \rho_{1}^{i}+c_{2} \rho_{2}^{i}+c_{3} \rho_{3}^{i}$, then the $i^{\text {th }}$ pair of coefficients in $\operatorname{gr}(B)$ is given by

$$
a_{i}=\frac{1}{2}\left(c_{1}+c_{2}-c_{3}\right), \quad b_{i}=\frac{1}{2}\left(-c_{1}+c_{2}+c_{3}\right) .
$$

To define the gradings on a bordered multimodule in a given $\operatorname{spin}^{c}$-structure, we choose a base generator $\boldsymbol{x}$ in that $\operatorname{spin}^{c}$-structure. Let $\mathcal{P}(\boldsymbol{x})$ be the subgroup of $G_{n, m}$ generated by $\left\{\operatorname{gr}(B) \mid B \in \pi_{2}(\boldsymbol{x}, \boldsymbol{x})\right\}$. Then $\widehat{\mathrm{CF}}(\mathcal{H})$ has a well defined grading by the set $G_{n, m} / \mathcal{P}(\boldsymbol{x})$. Up to canonical isomorphism, this grading set does not depend on the choice of $\boldsymbol{x}$. We define the relative grading by the following rule: if $\boldsymbol{y}$ is generator in the same $\operatorname{spin}^{c}$-structure as $\boldsymbol{x}$ and $B$ is a domain connecting $\boldsymbol{x}$ to $\boldsymbol{y}$, then $\operatorname{gr}(\boldsymbol{y})=\operatorname{gr}(\boldsymbol{x}) \operatorname{gr}(B)$.

In many cases, gradings can be computed directly from the labeled graph representing $\widehat{\mathrm{CF}}(\mathcal{H})$, without reference to the Heegaard diagram. To do this, we use the fact that elements of the torus algebra have gradings in $G_{n, m}$. Recall that $\widehat{\mathrm{CF}}(\mathcal{H})$ is a module over $n+m$ copies of the torus algebra, one for each boundary of $Y$, and $\rho_{I}^{i}$ denotes an element of the torus algebra associated to the $i^{\text {th }}$ boundary. The Maslov component of $\operatorname{gr}\left(\rho_{I}^{i}\right)$ is $-\frac{1}{2}$ and the coefficients $a_{j}$ and $b_{j}$ are zero for all $j \neq i$. The coefficients $a_{i}$ and $b_{i}$ are determined by $I$ as follows:

\begin{tabular}{c|rc}
\hline$I$ & $a_{i}$ & $b_{i}$ \\
\hline 1 & $1 / 2$ & $-1 / 2$ \\
2 & $1 / 2$ & $1 / 2$ \\
3 & $-1 / 2$ & $1 / 2$ \\
12 & 1 & 0 \\
23 & 0 & 1 \\
123 & $1 / 2$ & $1 / 2$ \\
\hline
\end{tabular}

This grading respects the algebra product in the sense that $\operatorname{gr}\left(\rho_{I_{1}} \rho_{I_{2}}\right)=\operatorname{gr}\left(\rho_{I_{1}}\right) \operatorname{gr}\left(\rho_{I_{2}}\right)$. The grading on $\widehat{\mathrm{CF}}(\mathcal{H})$ also respects the module structure in the sense that $\operatorname{gr}\left(\rho_{I} x\right)=$ $\operatorname{gr}\left(\rho_{I}\right) \operatorname{gr}(x)$, where the product on the right refers to the left action of the group $G_{n, m}$ on the set $G_{n, m} / \mathcal{P}(\boldsymbol{x})$. Finally, the grading on $\widehat{\mathrm{CF}}(\mathcal{H})$ satisfies the following relation [10, Definition 2.5.2]:

$$
\operatorname{gr}\left(m_{k+1}\left(x, \rho_{I_{1}}, \ldots, \rho_{I_{k}}\right)\right)=\lambda^{k-1} \operatorname{gr}\left(\rho_{I_{k}}\right) \cdots \operatorname{gr}\left(\rho_{I_{1}}\right) \operatorname{gr}(x) .
$$

Here $\lambda=(1 ; 0,0 ; \ldots ; 0,0)$ is the preferred central element of $G_{n, m}$. The same relation applies for both type $D$ and type $A$ modules if we think of the differential $\partial$ as an $m_{1}$ map. Thus $\operatorname{gr}(\partial x)=\lambda^{-1} \operatorname{gr}(x)$. 
To compute the relative grading from the graph representing $\widehat{\mathrm{CF}}(\mathcal{H})$, we choose a base generator $\boldsymbol{x}$ and assign it an arbitrary grading. The gradings of the remaining generators can be determined using (7), as long as each generator is connected by $\boldsymbol{x}$ by a path of arrows (that is, as long as the graph is connected). A loop in the graph representing $\widehat{\mathrm{CF}}(\mathcal{H})$, along with $(7)$, gives rise to a value for $\operatorname{gr}(\boldsymbol{x})$ which may not be equal to the value initially chosen for $\operatorname{gr}(\boldsymbol{x})$. The difference is $\operatorname{gr}(B)$ for some periodic domain $B \in \pi_{2}(\boldsymbol{x}, \boldsymbol{x})$. If there are enough loops in the graph (there must be one independent periodic domain for each boundary component of $Y$ ), then we can determine $\mathcal{P}(\boldsymbol{x})$.

\section{Direct computation of $\widehat{\mathbf{C F D}}^{k}\left(\mathcal{Y}_{\mathcal{P}}\right)$}

In this section we explicitly compute the type $D$ trimodule associated to $\mathcal{Y}_{\mathcal{P}}$, the trivial $S^{1}$-bundle over the pair of paints $\mathcal{P}$.

\section{A Choosing a bordered Heegaard diagram for $\mathcal{Y}_{\mathcal{P}}$}

We obtain the Heegaard surface $\Sigma$ from the boundary of $\mathcal{Y}_{\mathcal{P}}$ by drilling tubes through $\mathcal{Y}_{\mathcal{P}}$ to connect one boundary component to the other two. This surface is pictured in Figure 4, where the front and back faces are identified by the identity map. To obtain the 3-manifold $\mathcal{Y}_{\mathcal{P}}$ from this surface, we attach three 2-handles to the inside along the $\beta$ curves, and fill in the drilling tubes by attaching disks along the thick gray dashed lines. Filling in the interior with a 3 -ball yields $\mathcal{Y}_{\mathcal{P}}$.

We decorate each boundary component of $\mathcal{Y}_{\mathcal{P}}$ with a pair of intersecting $\alpha$ curves to specify a parametrization of the boundary. There are many choices of parametrization, but it is natural and convenient to choose one $\alpha$ curve to lie in the base surface $\mathcal{P}$ and the other to be an $S^{1}$ fiber.

To finish the construction of $\mathcal{H}$ we must also remove a disk around each $\alpha-\alpha$ intersection point, turning the $\alpha$ curves into arcs and giving the surface $\Sigma$ three boundary components, and we must chose a basepoint $z$ connected by arcs to each component of $\partial \Sigma$. These features are not shown until after the surface has been simplified by isotopy in Figures 5 and 6 . Notice the placement of the $\alpha$ curves relative to the drilling tunnels in Figure 4. This was to ensure that there is one component of $\Sigma \backslash(\boldsymbol{\alpha} \cup \boldsymbol{\beta})$ that meets all three components of $\partial \Sigma$.

The bordered Heegaard diagram $\mathcal{H}$ in Figure 6 can be represented in the plane (except for the handles) by cutting $\Sigma$ open along the $\operatorname{arcs}$ in $z$. The result is shown in Figure 7, and some relevant labels have been added. The Reeb chords along the three boundary 


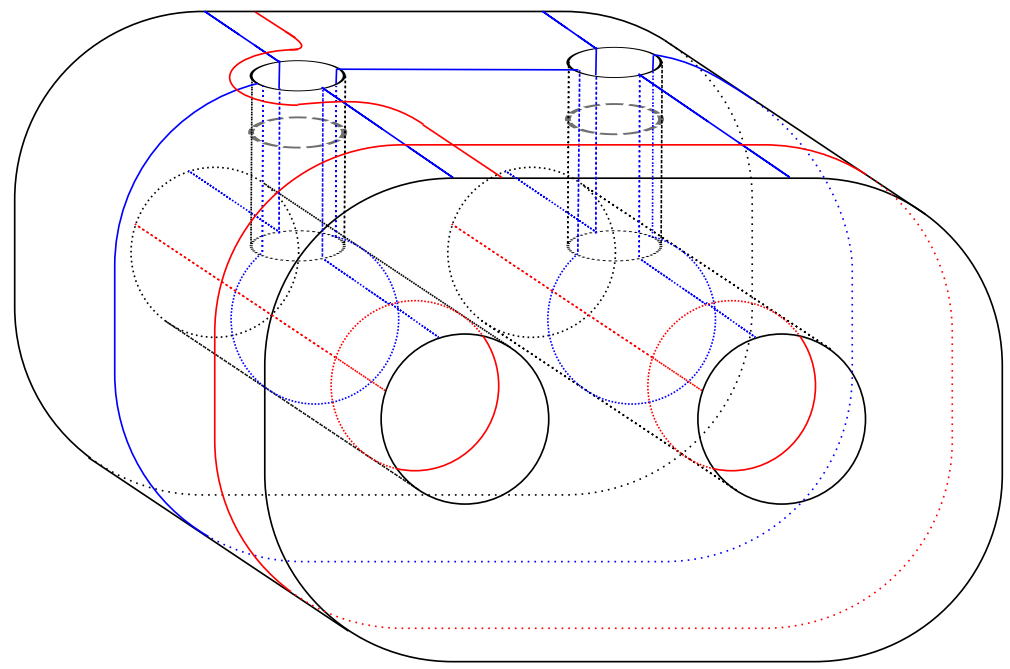

Figure 4: Constructing the Heegaard diagram for $\mathcal{Y}_{\mathcal{P}}$. The front and back faces are identified. Attaching 2-handles to the $\beta$ curves (blue), capping off the drilling tubes along the dashed gray lines, and adding a 3 -handle yields $\mathcal{Y}_{\mathcal{P}}$. Intersecting pairs of $\alpha$ curves (red) specify a parametrization of each boundary component of $\mathcal{Y}_{\mathcal{P}}$.

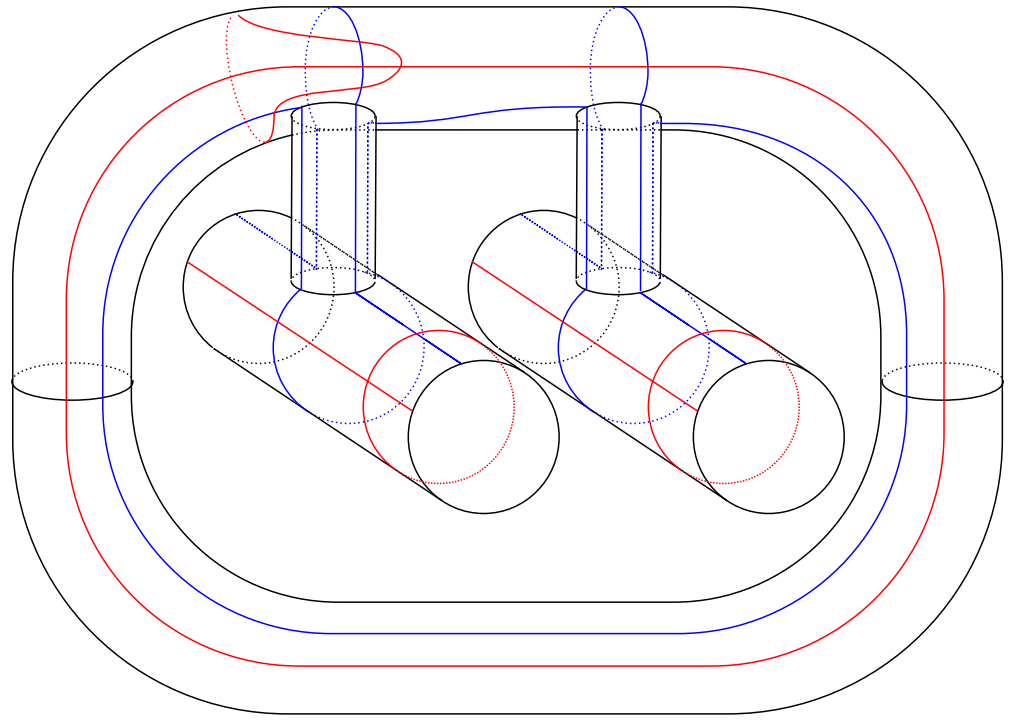

Figure 5: The diagram is easier to read and manipulate if we redraw the outer torus. 


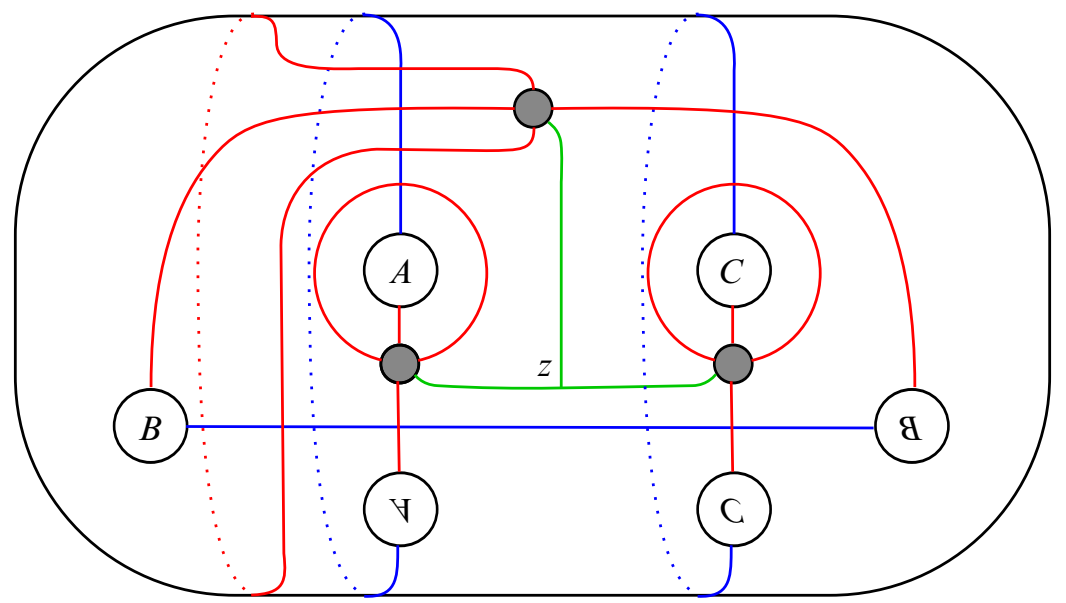

Figure 6: An isotopy simplifies the diagram. Pairs of circles labeled by letters signify 1-handle attachment. We remove a small disk (shaded) around each intersection of $\alpha$ curves, resulting in the genus-3 Heegaard surface $\Sigma$ with three boundary components. There is a basepoint $z$ connected by arcs (green) to each boundary component.

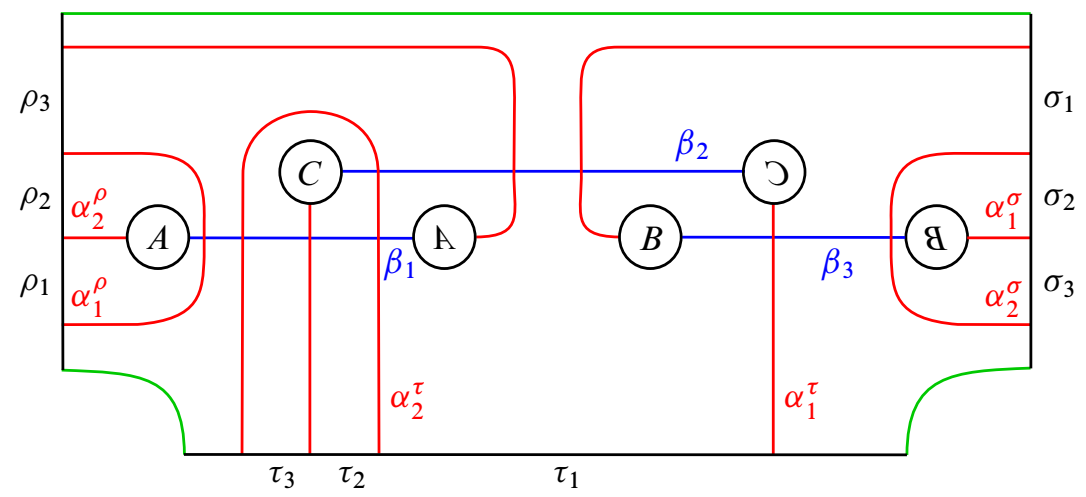

Figure 7: A bordered Heegaard diagram $\mathcal{H}$ for $\mathcal{Y}_{\mathcal{P}}$, with type $D$ boundaries

components are labeled in the order consistent with type $D$ boundaries, and they are denoted by $\rho, \sigma$ and $\tau$ instead of $\rho^{1}, \rho^{2}$ and $\rho^{3}$. The $\alpha$ arcs are also labeled to correspond to type $D$ boundaries. Tracing through the sequence in Figures 4 through 7 with $\alpha$ labels in mind, note that the arcs $\alpha_{1}^{\rho}, \alpha_{2}^{\sigma}$ and $\alpha_{1}^{\tau}$ represent curves in the base surface $\mathcal{P}$ of the $S^{1}$-bundle $\mathcal{Y}_{\mathcal{P}}$, and the $\operatorname{arcs} \alpha_{2}^{\rho}, \alpha_{1}^{\sigma}$ and $\alpha_{2}^{\tau}$ represent $S^{1}$ fibers.

Before computing $\widehat{\mathrm{CFD}}^{3}\left(\mathcal{Y}_{\mathcal{P}}\right)$, we make one final adjustment to the Heegaard diagram $\mathcal{H}$. Computing directly from $\mathcal{H}$ would involve a few large and complicated 


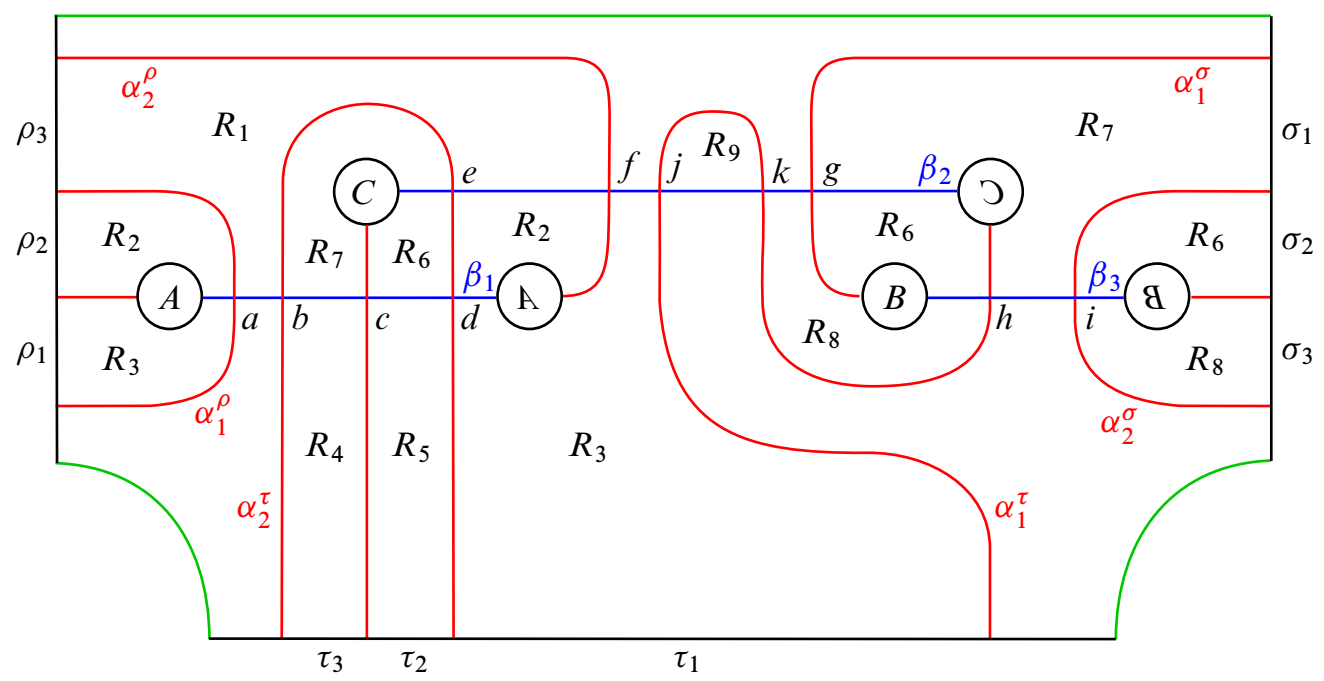

Figure 8: A slightly modified arced Heegaard diagram for $\mathcal{Y}_{\mathcal{P}}, \mathcal{H}^{\prime}$

domains which are difficult to analyze. It will be convenient to perform an isotopy to produce the new Heegaard diagram $\mathcal{H}^{\prime}$ shown in Figure 8. This change introduces a few extra generators, but it eliminates the trickiest domains and overall makes the computation easier.

\section{B Complex structure}

To compute $\widehat{\mathrm{CFD}}^{3}$, we also must fix a generically chosen complex structure $J$ on $\Sigma$. We collect here some relevant choices about $J$ that will be used in the computation. Note that if $J$ were chosen with different properties we would get a different, but quasi-isomorphic, trimodule.

As in the proof of Proposition 2.6, if the annulus $A$ has one $\alpha$ and one $\beta$ segment on each boundary component, let $\Theta_{A}^{p, q}$ denote the ratio of the lengths of the $\alpha$ and $\beta$ segments on the boundary component which contains $p$ and $q$. As shorthand we will use, for example, the subscript " 67 " for the annulus $R_{6} R_{7}$. We will assume that $J$ satisfies:

- $\Theta_{67}^{i, i}>\Theta_{67}^{d, b}$;

- $\Theta_{1267}^{i, i}>\Theta_{1267}^{a, a}$.

We will also assume that the following arcs are sufficiently short, in the sense of Proposition 2.7: 


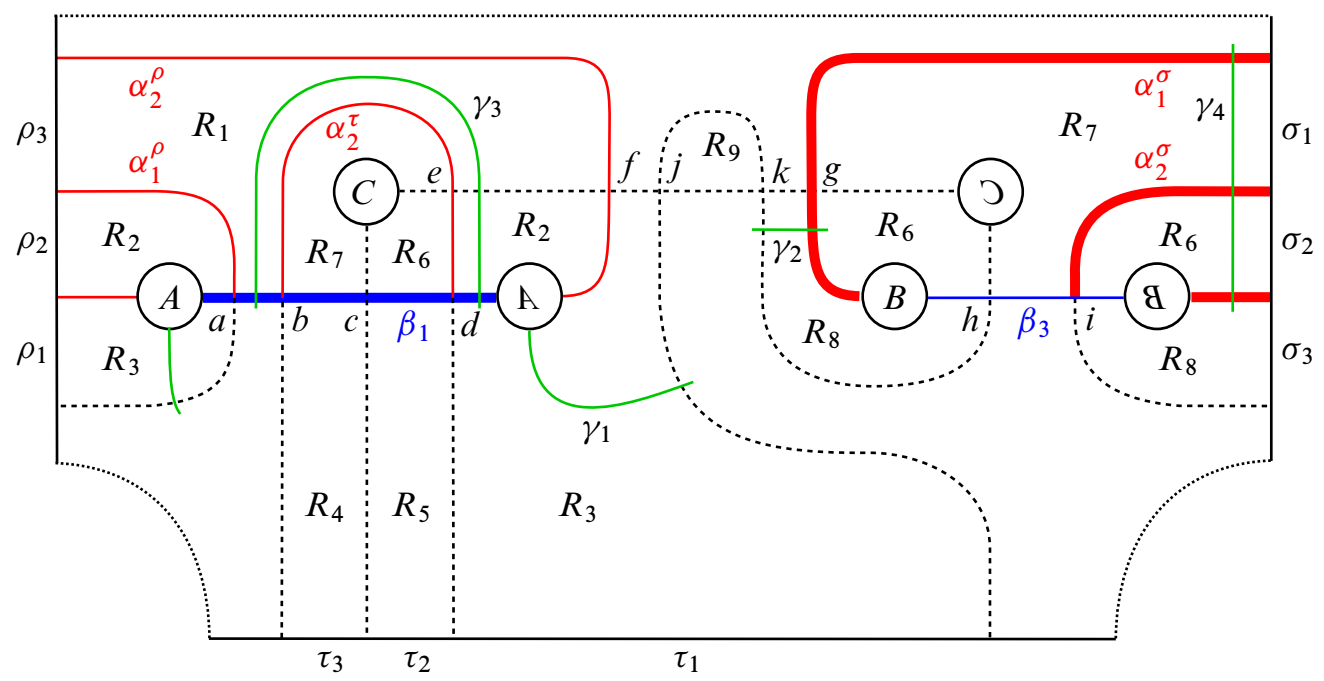

Figure 9: We choose a complex structure $J$ for which the four $\gamma$ arcs are very short and the bold $\alpha$ and $\beta$ segments are very long relative to the thin, solid $\alpha$ and $\beta$ segments.

- an arc $\gamma_{1}$ in $R_{3}$ from $\alpha_{1}^{\rho}$ to $\alpha_{1}^{\tau}$;

- an arc $\gamma_{2}$ in $R_{8}$ from $\alpha_{1}^{\sigma}$ to $\alpha_{1}^{\tau}$;

- an arc $\gamma_{3}$ in $R_{1} \cup R_{2}$ parallel to $\alpha_{2}^{\tau}$, from $\beta_{1}$ to itself.

These arcs are pictured in Figure 9.

We need to check that there exist suitable complex structures $J$ consistent with these assumptions. Starting with an arbitrary complex structure, we can modify it in regions $R_{6}$ and $R_{7}$ so that $\Theta_{67}^{i, i}>\Theta_{67}^{d, b}$ and $\Theta_{1267}^{i, i}>\Theta_{1267}^{a, a}$. This can be done, for instance, by pinching an arc parallel to the $\sigma$ boundary, transverse to $\alpha_{1}^{\sigma}$ and $\alpha_{2}^{\sigma}\left(\gamma_{4}\right.$ in Figure 9). Modifying the complex structure in a neighborhood of this arc as in the proof of Proposition 2.7 makes curves transverse to $\gamma_{4}$ very long and ensures that $\Theta_{67}^{i, i}$ and $\Theta_{1267}^{i, i}$ are large. Once $\gamma_{4}$ is sufficiently pinched, we pinch the $\operatorname{arcs} \gamma_{1}, \gamma_{2}$ and $\gamma_{3}$ in the same way. Note that as a side effect of pinching these arcs, $\beta_{1}$ and $\alpha_{1}^{\sigma}$ get longer, but this only increases $\Theta_{67}^{i, i}$ and $\Theta_{1267}^{i, i}$ and decreases $\Theta_{1267}^{a, a}$.

Note that each assumption we make on $J$ can be satisfied, individually, by pinching an arc in the Heegaard diagram. All the assumptions can be satisfied consistently as long as all the arcs that need to be pinched are disjoint and none of them is transverse to an $\alpha$ or $\beta$ segment that is supposed to be short relative to other segments; see Figure 9. 


\section{C Generators}

The chain complex $\widehat{\mathrm{CFD}}^{3}\left(\mathcal{H}^{\prime}\right)$ is generated by the set $\mathfrak{S}\left(\mathcal{H}^{\prime}\right)$ consisting of triples of intersection points with one point on each $\beta$ circle and at most one point on each $\alpha$ arc. In total, there are 23 generators. These generators fall into seven different $\operatorname{spin}^{c}-$ structures, corresponding to how many $\alpha$ arcs are occupied on each boundary component.

We begin by computing the summand of $\widehat{\mathrm{CFD}}^{3}\left(\mathcal{H}^{\prime}\right)$ corresponding to the middle spin ${ }^{c_{-}}$ structure, with exactly one $\alpha$ arc occupied on each boundary (the other $\operatorname{spin}^{c}$-structures are much easier and will be addressed at the end of this section). There are seven generators in the middle $\operatorname{spin}^{c}$-structure: $x=(a, e, i), y=(a, g, h), z=(c, f, i)$, $v=(b, f, i), w=(d, f, i), s=(a, j, i)$ and $t=(a, k, i)$.

\section{D Possible domains}

We begin by listing domains in $\pi_{2}(\boldsymbol{x}, \boldsymbol{y})$ for any pair of generators $\boldsymbol{x}$ and $\boldsymbol{y}$. Recall that a domain connecting $\boldsymbol{x}$ and $\boldsymbol{y}$ is a linear combination of the regions $R_{1}, \ldots, R_{9}$ in Figure 8 with the correct multiplicity at each corner (that is, satisfying (4)). We do not need to list every domain in $\pi_{2}(\boldsymbol{x}, \boldsymbol{y})$, since only positive domains can have holomorphic representatives, and by Proposition 2.3 we can assume that regions which intersect $\partial \Sigma$ have multiplicity 0 or 1 . The only region which does not intersect the boundary is $R_{9}$. The multiplicity of $R_{9}$ is also limited; in fact, $R_{9}$ can not combine with any other regions because domains with holomorphic representatives must be connected in $\Sigma \backslash(\alpha \cap \beta)$. Thus we can restrict to linear combinations of $R_{1}, \ldots, R_{8}$ with multiplicity 0 or 1 for each region, and the single domain $R_{9}$. All such domains which connect two generators are listed in Table 1.

\section{E Compatibility and idempotents}

Several domains in Table 1 can be ruled out using Proposition 2.3. Consider for example the domain $R_{2} R_{3}$, which potentially contributes to the differential from $x$ to $s$. By Proposition 2.3, this domain can only contribute with the Reeb chords $\left(\rho_{1}, \rho_{2}\right)$, and then the contribution $a\left(\rho_{12}\right) \otimes s$ is zero unless $s$ contains a point on $\alpha_{2}^{\rho}$. Since $s$ does not contain a point on $\alpha_{2}^{\rho}$, the domain $R_{2} R_{3}$ has zero contribution to the differential.

In general, for a differential ending in $s$ to be nontrivial, the algebra element for the $\rho$ boundary can not be $\rho_{2}$ or $\rho_{12}$. This means that the domain associated with such a differential can not contain $R_{2}$ without containing $R_{1}$. In addition to $R_{2} R_{3}$, this line of reasoning eliminates the domains $R_{2} R_{3} R_{4} R_{5} R_{6} R_{7}, R_{2} R_{3} R_{5} R_{6}$ and $R_{2} R_{3} R_{5} R_{6} R_{8}$. A similar analysis on the other boundaries shows that domains 


\begin{tabular}{|c|c|c|c|c|c|c|c|}
\hline to $\downarrow$ from $\rightarrow$ & $x$ & $y$ & $z$ & $w$ & $v$ & $s$ & $t$ \\
\hline$x$ & $\begin{array}{l}1267 \\
4567\end{array}$ & 56 & 1567 & 167 & $\begin{array}{c}1 \\
14567\end{array}$ & - & 568 \\
\hline$y$ & 47 & $\begin{array}{l}1267 \\
4567\end{array}$ & 17 & - & 147 & - & $\begin{array}{c}812678 \\
45678\end{array}$ \\
\hline$z$ & 2467 & 26 & $\begin{array}{l}1267 \\
4567\end{array}$ & 467 & $\begin{array}{c}4 \\
12467\end{array}$ & - & 268 \\
\hline$w$ & $\begin{array}{c}2 \\
24567\end{array}$ & 256 & $\begin{array}{c}5 \\
12567\end{array}$ & $\begin{array}{l}1267 \\
4567\end{array}$ & $\begin{array}{c}1245 \\
124567\end{array}$ & - & 2568 \\
\hline$v$ & 267 & - & 567 & 67 & $\begin{array}{l}1267 \\
4567\end{array}$ & - & - \\
\hline$S$ & $\begin{array}{c}23 \\
234567\end{array}$ & 2356 & $\begin{array}{c}35 \\
123567\end{array}$ & $\begin{array}{c}312367 \\
34567\end{array}$ & $\begin{array}{l}123 \quad 345 \\
1234567\end{array}$ & $\begin{array}{l}1267 \\
4567\end{array}$ & $\begin{array}{c}23568 \\
9\end{array}$ \\
\hline$t$ & - & - & - & - & - & - & $\begin{array}{l}1267 \\
4567\end{array}$ \\
\hline
\end{tabular}

Table 1: Domains which potentially contribute to the differential. All subsets of the regions $\left\{R_{1}, \ldots, R_{8}\right\}$ with the proper corner multiplicities, and the single domain $R_{9}$. We omit the $R$ for the purposes of this table. Thus 56 refers to the domain $R_{5} R_{6}$, which connects $y$ to $x$.

contributing nontrivial differentials ending in $s$ cannot contain $R_{5}$ without $R_{4}, R_{7}$ without $R_{6}$, or $R_{8}$. This further rules out the domains $R_{3} R_{5}$ and $R_{1} R_{2} R_{3} R_{5} R_{6} R_{7}$. Finally, applying the same technique to differentials ending in other generators rules out the following domains:

domains to $x$ : $4567,14567,568$;

domains to $y$ : 1267,4567 ;

domains to $z: 1267,12467,268$;

domains to $w: 24567,12567,1267,4567,12,45,124567,2568$;

domains to $v: 1267,4567$.

\section{F Polygons}

Of the remaining domains from Table 1, many are immersed polygons and therefore contribute to the differential by Proposition 2.5. The proposition depends on the sequence of Reeb chords $\vec{\rho}$, but each of the following domains has only one compatible sequence of Reeb chords, so Proposition 2.5 tells us the entire contribution of the 
domain to the differential:

$\begin{array}{cccccc}R_{1} & \text { contributes } & v \stackrel{\rho_{3}}{\longrightarrow} x & R_{2} & \text { contributes } & x \stackrel{\rho_{2}}{\longrightarrow} w \\ R_{3} & \text { contributes } & w \stackrel{\rho_{1} \tau_{1}}{\longrightarrow} s & R_{4} & \text { contributes } & v \stackrel{\tau_{3}}{\longrightarrow} z \\ R_{5} & \text { contributes } & z \stackrel{\tau_{2}}{\longrightarrow} w & R_{8} & \text { contributes } & t \stackrel{\sigma_{3}}{\longrightarrow} y \\ R_{9} & \text { contributes } & t \stackrel{1}{\longrightarrow} s & R_{1} R_{7} & \text { contributes } & z \stackrel{\rho_{3} \sigma_{1}}{\longrightarrow} y \\ R_{2} R_{6} & \text { contributes } & y \stackrel{\rho_{2} \sigma_{2}}{\longrightarrow} z & R_{4} R_{7} & \text { contributes } & x \stackrel{\tau_{3} \sigma_{1}}{\longrightarrow} y \\ R_{5} R_{6} & \text { contributes } & y \stackrel{\tau_{2} \sigma_{2}}{\longrightarrow} x & & & \end{array}$

Here the notation $\boldsymbol{x} \stackrel{a}{\rightarrow} \boldsymbol{y}$ means that there is an $a \otimes \boldsymbol{y}$ term in $\partial \boldsymbol{x}$.

\section{G Index}

At this point there are 22 domains in Table 1 whose contribution to $\widehat{\mathrm{CFD}}^{k}$ remain unknown. Of these, 11 can be ruled out by showing that $\operatorname{ind}(B) \neq 1$. In general computing the index is a good task for a computer, but because we have narrowed the list of domains down so much we can work out the index computations by hand.

The quantities $e(B)$ and $n_{\boldsymbol{x}}(B)$ for any generator $\boldsymbol{x}$ are additive, so it is helpful to record their values for individual regions; see Table 2. For instance, region $R_{1}$ has Euler measure $e\left(R_{1}\right)=-\frac{1}{2}$, because it has Euler characteristic $\chi\left(R_{1}\right)=1$ and six acute corners. $R_{1}$ has a corner at the point $a$, which means that the average multiplicity of $R_{1}$ near $a$ is $\frac{1}{4}$. Also, $R_{1}$ has corners at $b, e$ and $f$. For the generator $x=(a, e, i)$ we find that $n_{x}\left(R_{1}\right)=\frac{1}{4}+\frac{1}{4}+0=\frac{1}{2}$, and for $y=(a, g, h)$ we get $n_{y}\left(R_{1}\right)=\frac{1}{4}+0+0=\frac{1}{4}$. It is straightforward to fill in the rest of Table 2 .

\begin{tabular}{l|rrrrrrrc}
\hline & $e$ & $n_{x}$ & $n_{y}$ & $n_{z}$ & $n_{v}$ & $n_{w}$ & $n_{s}$ & $n_{t}$ \\
\hline$R_{1}$ & $-1 / 2$ & $1 / 2$ & $1 / 4$ & $1 / 4$ & $1 / 2$ & $1 / 4$ & $1 / 4$ & $1 / 4$ \\
$R_{2}$ & $-1 / 2$ & $1 / 2$ & $1 / 4$ & $1 / 4$ & $1 / 4$ & $1 / 2$ & $1 / 4$ & $1 / 4$ \\
$R_{3}$ & -1 & $1 / 4$ & $1 / 4$ & $1 / 4$ & $1 / 4$ & $1 / 2$ & $1 / 2$ & $1 / 4$ \\
$R_{4}$ & 0 & 0 & 0 & $1 / 4$ & $1 / 4$ & 0 & 0 & 0 \\
$R_{5}$ & 0 & 0 & 0 & $1 / 4$ & 0 & $1 / 4$ & 0 & 0 \\
$R_{6}$ & -1 & $1 / 2$ & $1 / 2$ & $1 / 2$ & $1 / 4$ & $1 / 2$ & $1 / 4$ & $1 / 4$ \\
$R_{7}$ & -1 & $1 / 2$ & $1 / 2$ & $1 / 2$ & $1 / 2$ & $1 / 4$ & $1 / 4$ & $1 / 4$ \\
$R_{8}$ & $-1 / 2$ & $1 / 4$ & $1 / 2$ & $1 / 4$ & $1 / 4$ & $1 / 4$ & $1 / 4$ & $1 / 2$ \\
\hline
\end{tabular}

Table 2 


\begin{tabular}{cl|cccc|c}
\hline Domain & $\boldsymbol{x} \rightarrow \boldsymbol{y}$ & $e(B)$ & $n_{\boldsymbol{x}}$ & $n_{\boldsymbol{y}}$ & bdys hit/2 & ind( $B)$ \\
\hline 1267 & $x \rightarrow x$ & -3 & 2 & 2 & 1 & 2 \\
2467 & $x \rightarrow z$ & $-5 / 2$ & $3 / 2$ & $3 / 2$ & $3 / 2$ & 2 \\
256 & $y \rightarrow w$ & $-3 / 2$ & $3 / 4$ & $5 / 4$ & $3 / 2$ & 2 \\
1567 & $z \rightarrow x$ & $-5 / 2$ & $3 / 2$ & $3 / 2$ & $3 / 2$ & 2 \\
4567 & $z \rightarrow z$ & -2 & $3 / 2$ & $3 / 2$ & 1 & 2 \\
147 & $v \rightarrow y$ & $-3 / 2$ & $5 / 4$ & $3 / 4$ & $3 / 2$ & 2 \\
67 & $w \rightarrow v$ & -2 & $3 / 4$ & $3 / 4$ & $1 / 2$ & 0 \\
1267 & $s \rightarrow s$ & -3 & 1 & 1 & 1 & 0 \\
4567 & $s \rightarrow s$ & -2 & $1 / 2$ & $1 / 2$ & 1 & 0 \\
1267 & $t \rightarrow t$ & -3 & 1 & 1 & 1 & 0 \\
4567 & $t \rightarrow t$ & -2 & $1 / 2$ & $1 / 2$ & 1 & 0 \\
\hline
\end{tabular}

Table 3

From this information, it is easy to compute the index as in Table 3. We add the Euler measures of all of the regions in a given domain $B$ to find $e(B)$. Similarly, we add the values of $n_{\boldsymbol{x}}$ and $n_{\boldsymbol{y}}$ for each region for the relevant generators $\boldsymbol{x}$ and $\boldsymbol{y}$ to find $n_{\boldsymbol{x}}(B)$ and $n_{\boldsymbol{y}}(B)$. Finally, we count how many of the three components of $\partial \Sigma$ are touched by $B$ (that is, we find $\#\left\{Z \in \pi_{0}(\partial \Sigma) \mid Z \cap B \neq \varnothing\right\}$ ), and add half of this number to $e(B)+n_{\boldsymbol{x}}(B)+n_{\boldsymbol{y}}(B)$. By (6), the result is ind $(B)$. Table 3 only shows the computation for regions that are ruled out by this method. The index can be computed in the same way for the remaining 11 domains, but they all have $\operatorname{ind}(B)=1$, so more work is needed to determine if they contribute to $\widehat{\mathrm{CFD}}^{k}$.

\section{H Index-zero annulus}

$R_{6} R_{7}$ is an index-0 annulus of the same form as $A$ in Proposition 2.6; see Figure 10. The domains $R_{2} R_{6} R_{7}, R_{5} R_{6} R_{7}, R_{1} R_{6} R_{7}$ and $R_{4} R_{6} R_{7}$ in $\mathcal{H}^{\prime}$ correspond to the domains $D_{1}, D_{2}, D_{3}$ and $D_{4}$, respectively, in Figure 2. By Proposition 2.6, the contribution of these four domains is determined by the choice of complex structure $J$ on $\Sigma$, and in particular on the resulting ratios of lengths $\Theta_{67}^{i, i}$ and $\Theta_{67}^{d, b}$. Recall that we chose the complex structure on $\Sigma$ so that $\Theta_{67}^{i, i}>\Theta_{67}^{d, b}$.

It follows directly from the proof of Proposition 2.6 that $R_{4} R_{6} R_{7}$ and $R_{5} R_{6} R_{7}$ contribute to the differential for our choice of $J$. Specifically, $R_{4} R_{6} R_{7}$ contributes $\tau_{3} \sigma_{12} \otimes z$ to $\partial w$ and $R_{5} R_{6} R_{7}$ contributes $\tau_{2} \sigma_{12} \otimes v$ to $\partial z$. It is also true that $R_{1} R_{6} R_{7}$ and $R_{2} R_{6} R_{7}$ do not contribute to the differential, but for these domains the outer boundary of the annulus has too many $\alpha$ and $\beta$ segments to apply Proposition 2.6 


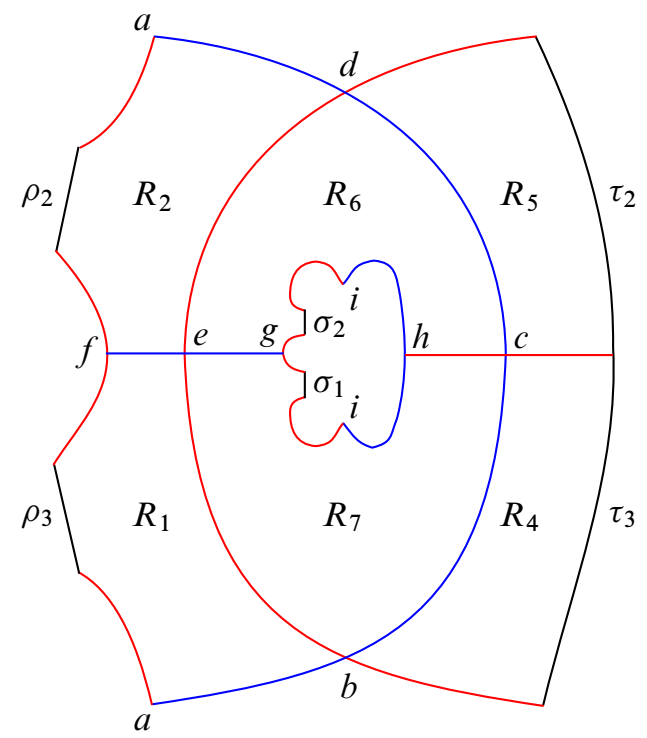

Figure 10: The index- 0 annulus $R_{6} R_{7}$, with four surrounding regions

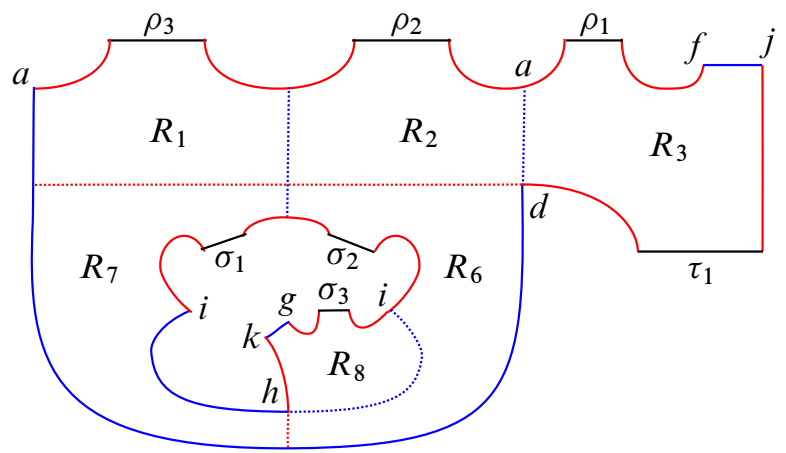

Figure 11: The annulus $R_{1} R_{2} R_{6} R_{7} R_{8}$ contributes to the differential, while $R_{1} R_{2} R_{3} R_{6} R_{7}$ does not.

directly. First we apply Proposition 2.7 and pinch along the $\operatorname{arc} \gamma_{3}$ in $R_{1}$ and $R_{2}$; recall that the complex structure was chosen to be consistent with pinching this arc. The annuli obtained from $R_{1} R_{6} R_{7}$ and $R_{2} R_{6} R_{7}$ by pinching this arc completely do not have representatives by Proposition 2.6, and so $R_{1} R_{6} R_{7}$ and $R_{2} R_{6} R_{7}$ do not contribute to the differential.

We need to perform a similar analysis on two more domains, which are obtained from adding regions to the index- 0 annulus $R_{1} R_{2} R_{6} R_{7}$; see Figure 11 . If we let the 
inner boundary in Figure 11 correspond to the outer boundary in Figure 2, then $R_{8}$ is analogous to $B_{4}$. However, $R_{8}$ is not a bigon, so we must first use Proposition 2.7 to obtain that the contribution of $R_{1} R_{2} R_{6} R_{7} R_{8}$ is the same as the contribution of the annulus which would result from collapsing the arc $\gamma_{2}$ in $R_{8}$. This pinched annulus would not contribute with the Reeb chords $\left(\rho_{2}, \rho_{3}, \sigma_{1}, \sigma_{2}, \sigma_{3}\right)$ by Proposition 2.6, using the fact that $\Theta_{1267}^{i, i}>\Theta_{1267}^{a, a}$. Thus the pair $\left(R_{1} R_{2} R_{6} R_{7} R_{8},\left(\rho_{2}, \rho_{3}, \sigma_{1}, \sigma_{2}, \sigma_{3}\right)\right)$ does not contribute to $\widehat{\mathrm{CFD}}^{k}$. We emphasize however that this domain has a second compatible sequence of Reeb chords, about which Proposition 2.6 says nothing. The contribution of this domain with $\vec{\rho}=\left(\rho_{2}, \rho_{3}, \sigma_{123}\right)$ will be discussed in Section 3I.

The domain $R_{1} R_{2} R_{3} R_{6} R_{7}$, with Reeb chords $\left(\rho_{1}, \rho_{2}, \rho_{3}, \sigma_{1}, \sigma_{2}, \tau_{1}\right)$, can be analyzed in a similar way. By Proposition 2.7 we will treat $R_{3}$ as a bigon attached to $R_{1} R_{2} R_{6} R_{7}$, since the extra $\beta$ segment can be removed by pinching along $\gamma_{1}$. In this case Proposition 2.6 does not apply, because the arc $\alpha_{2}^{\tau}$ cutting into the annulus from the obtuse corner does not leave the annulus on the opposite boundary component, but the reasoning is similar. There is a one parameter family of cuts starting at $d$. We can define the ratios $\theta_{12367}^{i, i}$ and $\theta_{12367}^{d, a}$, which depend on the cutting parameter, as in the proof of Proposition 2.6. There is a holomorphic representative for each zero of $\theta_{12367}^{i, i}-\theta_{12367}^{d, a}$. Cutting along $\alpha_{2}^{\tau}$ from $d$ to $b$ pinches off the annulus $R_{6} R_{7}$. In this extreme, $\theta_{12367}^{i, i}-\theta_{12367}^{d, a}$ approaches $\Theta_{67}^{i, i}-\Theta_{67}^{d, b}$, which is positive for our choice of complex structure. In the other extreme, cutting along $\beta_{1}$ from $d$ to $a$ pinches off the annulus $R_{1} R_{2} R_{6} R_{7}$, and $\theta_{12367}^{i, i}-\theta_{12367}^{d, a}$ becomes $\Theta_{1267}^{i, i}-\Theta_{1267}^{a, a}>0$. Since $\theta_{12367}^{i, i}-\theta_{12367}^{d, a}$ has the same sign at both extremes, the number of zeros is even and the pair $\left(R_{1} R_{2} R_{3} R_{6} R_{7},\left(\rho_{1}, \rho_{2}, \rho_{3}, \sigma_{1}, \sigma_{2}, \tau_{1}\right)\right)$ does not contribute to the differential.

\section{I Decomposable boundaries}

We have determined the contribution of all domains in Table 1 except for the following seven:

$\begin{array}{ccccc}R_{1} R_{2} R_{3} & \text { from } & v & \text { to } & s \\ R_{3} R_{4} R_{5} & \text { from } & v & \text { to } & s \\ R_{1} R_{2} R_{3} R_{6} R_{7} & \text { from } & w & \text { to } & s \\ R_{3} R_{4} R_{5} R_{6} R_{7} & \text { from } & w & \text { to } & s \\ R_{1} R_{2} R_{6} R_{7} R_{8} & \text { from } & t & \text { to } & y \\ R_{4} R_{5} R_{6} R_{7} R_{8} & \text { from } & t & \text { to } & y \\ R_{1} R_{2} R_{3} R_{4} R_{5} R_{6} R_{7} & \text { from } & v & \text { to } & s\end{array}$

Each of these domains is compatible with multiple Reeb chord sequences. The contribution of each domain/Reeb chord pair must be considered separately. 

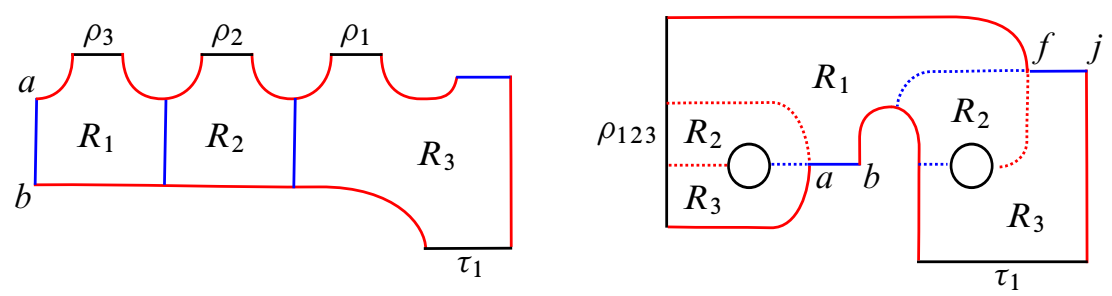

Figure 12: Two versions of the domain $R_{1} R_{2} R_{3}$. The left represents the sequence of Reeb chords $\vec{\eta}_{1}=\left(\rho_{3}, \rho_{2}, \rho_{1}, \tau_{1}\right)$. This domain/Reeb chord pair is a polygon and contributes to the differential. The right represents the sequence of Reeb chords $\vec{\eta}_{2}=\left(\rho_{123}, \tau_{1}\right)$. This genus-one domain also contributes to the differential.

$\boldsymbol{R}_{\mathbf{1}} \boldsymbol{R}_{\mathbf{2}} \boldsymbol{R}_{\mathbf{3}}$ The domain $R_{1} R_{2} R_{3}$ is compatible with both $\vec{\eta}_{1}=\left(\rho_{1}, \rho_{2}, \rho_{3}, \tau_{1}\right)$ and $\vec{\eta}_{2}=\left(\rho_{123}, \tau_{1}\right)$. By cutting along $\alpha$ arcs, the domain can be represented differently for each Reeb chord sequence; see Figure 12. On the left is an immersed polygon; it is clear that the conditions of Proposition 2.5 are satisfied, and so the pair $\left(R_{1} R_{2} R_{3}, \vec{\eta}_{1}\right)$ contributes to the differential.

For $\vec{\eta}_{2}$, we use Lemma 2.8 and consider the $\mathcal{A}_{\infty}$ relation for $\left(v, \rho_{1}, \rho_{23}, \tau_{3}\right)$ :

$$
0=m\left(v, \mu\left(\rho_{1}, \rho_{23}\right), \tau_{3}\right)+m\left(m\left(v, \rho_{1}\right), \rho_{23}, \tau_{3}\right) .
$$

There are no other nonzero terms in the relation. Note that it is impossible to have an $\mathcal{A}_{\infty}$ operation involving $\tau_{3}$ and not $\rho_{3}$, since both Reeb chords are on the same region $R_{3}$. Thus the term $m\left(m\left(v, \rho_{1}, \tau_{3}\right), \rho_{23}\right)$ does not appear in the $\mathcal{A}_{\infty}$ relation. Since we use $\mathbb{F}_{2}$ coefficients, the relation above can be rewritten as

$$
m\left(v, \rho_{123}, \tau_{3}\right)=m\left(m\left(v, \rho_{1}\right), \rho_{23}, \tau_{3}\right) .
$$

The inner operation on the right, $m\left(v, \rho_{1}\right)$, records the contribution of the domain $R_{1}$ with $\vec{\rho}=\left(\rho_{1}\right)$. We showed that this pair contributes in $\widehat{\mathrm{CFD}}^{3}$, and so by Lemma 2.8 it also contributes to $\widehat{\mathrm{CFA}}^{3}$. Thus $m\left(v, \rho_{1}\right)=x$. The outer operation is determined by the contribution of the domain $R_{2} R_{3}$. This domain was eliminated from consideration for $\widehat{\mathrm{CFD}}^{3}$, but it may still contribute to $\widehat{\mathrm{CFA}}^{3}$. To find out if it does we use another $\mathcal{A}_{\infty}$ relation, this time for $\left(x, \rho_{2}, \rho_{3}, \tau_{3}\right)$. The relation implies that

$$
m\left(x, \rho_{23}, \tau_{3}\right)=m\left(m\left(x, \rho_{2}\right), \rho_{3}, \tau_{3}\right) .
$$

Since $R_{2}$ and $R_{3}$ are known to contribute in $\widehat{\mathrm{CFD}}^{k}$ (and thus in $\widehat{\mathrm{CFA}}^{k}$ ), we find that $m\left(x, \rho_{23}, \tau_{3}\right)=m\left(w, \rho_{3}, \tau_{3}\right)=s$ and $m\left(v, \rho_{123}, \tau_{3}\right)=s$. By Lemma 2.8 the pair $\left(R_{1} R_{2} R_{3}, \vec{\eta}_{2}\right)$ contributes to $\widehat{\mathrm{CFD}}^{k}$. 
$\left(R_{1} R_{2} R_{3}, \vec{\eta}_{1}\right)$ and $\left(R_{1} R_{2} R_{3}, \vec{\eta}_{2}\right)$ both contribute the term $\rho_{123} \tau_{1} s$ to $\partial(v)$ in $\widehat{\mathrm{CFD}}^{k}$. Over $\mathbb{F}_{2}$, these contributions cancel, so the total contribution of $R_{1} R_{2} R_{3}$ to the differential is zero.

$\boldsymbol{R}_{\mathbf{3}} \boldsymbol{R}_{\mathbf{4}} \boldsymbol{R}_{\mathbf{5}}$ The two compatible Reeb chord sequences are $\vec{\eta}_{1}=\left(\rho_{1}, \tau_{1}, \tau_{2}, \tau_{3}\right)$ and $\vec{\eta}_{2}=\left(\rho_{1}, \tau_{123}\right)$. The first does not contribute, because a holomorphic representative consistent with $\vec{\eta}_{1}$ would project to $R_{3} R_{4} R_{5}$ with cuts along $\alpha_{1}^{\tau}$ and $\alpha_{2}^{\tau}$, making the domain disconnected. If we do not cut the domain along $\alpha_{1}^{\tau}$ and $\alpha_{2}^{\tau}$, we see that it is an immersed polygon compatible with $\vec{\eta}_{2}$, and Proposition 2.5 tells us that $\left(R_{3} R_{4} R_{5}, \vec{\eta}_{2}\right)$ contributes to the differential. Overall, the domain $R_{3} R_{4} R_{5}$ contributes $\rho_{1} \tau_{123} S$ to $\partial(v)$.

$\boldsymbol{R}_{\mathbf{1}} \boldsymbol{R}_{\mathbf{2}} \boldsymbol{R}_{\mathbf{3}} \boldsymbol{R}_{\mathbf{6}} \boldsymbol{R}_{\mathbf{7}}$ This domain is compatible with $\vec{\eta}_{1}=\left(\rho_{1}, \rho_{2}, \rho_{3}, \tau_{1}, \sigma_{1}, \sigma_{2}\right)$ and $\vec{\eta}_{2}=\left(\rho_{123}, \tau_{1}, \sigma_{1}, \sigma_{2}\right)$. It was shown in the previous section that there is no contribution from $\vec{\eta}_{1}$. For the other case consider the $A_{\infty}$ relation for $\left(w, \rho_{1}, \rho_{23}, \tau_{3}, \sigma_{3}, \sigma_{2}\right)$. To find all terms of the relation, we use Table 1 to split the domain $R_{1} R_{2} R_{3} R_{6} R_{7}$ into two pieces such that one is a domain connecting $w$ to some generator $\boldsymbol{x}$ and the other is a domain connecting $\boldsymbol{x}$ to $s$. There are two possibilities: the domain from $w$ can be $R_{1} R_{6} R_{7}$ or $R_{6} R_{7}$. This gives the $\mathcal{A}_{\infty}$ relation

$$
\begin{aligned}
m\left(w, \mu\left(\rho_{1}, \rho_{23}\right), \tau_{3}, \sigma_{3}, \sigma_{2}\right)=m\left(m\left(w, \rho_{1}, \sigma_{3}, \sigma_{2}\right), \rho_{23}, \tau_{3}\right) & \\
& +m\left(m\left(w, \sigma_{3}, \sigma_{2}\right), \rho_{1}, \rho_{23}, \tau_{3}\right) .
\end{aligned}
$$

The outer operation in the second term on the right is trivial for boundary monotonicity reasons. The inner operation on the of the first term is trivial because it records the contribution of the domain $R_{1} R_{6} R_{7}$. We have shown that this domain does not contribute to $\widehat{\mathrm{CFD}}^{3}$, and thus it also does not contribute to $\widehat{\mathrm{CFA}}^{3}$ by Lemma 2.8 . Thus $m\left(w, \rho_{123}, \tau_{3}, \sigma_{2}, \sigma_{1}\right)=0$ and $\left(R_{1} R_{2} R_{3} R_{6} R_{7}, \vec{\eta}_{2}\right)$ does not contribute to $\widehat{\mathrm{CFD}}^{3}$. The total contribution of $R_{1} R_{2} R_{3} R_{6} R_{7}$ is zero.

$\boldsymbol{R}_{\mathbf{3}} \boldsymbol{R}_{\mathbf{4}} \boldsymbol{R}_{\mathbf{5}} \boldsymbol{R}_{\mathbf{6}} \boldsymbol{R}_{\mathbf{7}}$ This domain is compatible with $\vec{\eta}_{1}=\left(\rho_{1}, \tau_{1}, \tau_{2}, \tau_{3}, \sigma_{1}, \sigma_{2}\right)$ and $\vec{\eta}_{2}=\left(\rho_{1}, \tau_{123}, \sigma_{1}, \sigma_{2}\right)$. There is no contribution from $\vec{\eta}_{1}$ because realizing the domain with boundary Reeb chords $\vec{\eta}_{1}$ would involve cutting along $\alpha_{2}^{\tau}$, which leaves the domain disconnected. For the contribution of $\vec{\eta}_{2}$, consider the $A_{\infty}$ relation for $\left(w, \rho_{3}, \tau_{12}, \tau_{3}, \sigma_{3}, \sigma_{2}\right)$. To find all terms of the relation, first note that any type $A$ operation that involves $m\left(\boldsymbol{x}, \ldots, \tau_{12}, \tau_{3}, \ldots\right)$ will be trivial for boundary monotonicity reasons. As a result, any term in the $\mathcal{A}_{\infty}$ relation splits as an operation involving $\tau_{12}$ and an operation involving $\tau_{3}$. We look in Table 1 for domains that connect $w$ to another generator and involve $R_{4}$ and $R_{5}$, but not $R_{3}$, and some subset of $\left\{R_{6}, R_{7}\right\}$. 
The only option is the domain $R_{4} R_{5} R_{6} R_{7}$ connecting $w$ to itself. The relation can be written as

$$
m\left(w, \rho_{3}, \tau_{123}, \sigma_{3}, \sigma_{2}\right)=m\left(m\left(w, \tau_{12}, \sigma_{3}, \sigma_{2}\right), \rho_{3}, \tau_{3}\right) .
$$

To compute the inner operation on the right, we can use another $\mathcal{A}_{\infty}$ relation to show that

$$
m\left(w, \tau_{12}, \sigma_{3}, \sigma_{2}\right)=m\left(m\left(w, \tau_{1}, \sigma_{3}, \sigma_{2}\right), \tau_{2}\right)=m\left(z, \tau_{2}\right)=w,
$$

where $m\left(w, \tau_{1}, \sigma_{3}, \sigma_{2}\right)$ is nontrivial because the domain $R_{4} R_{6} R_{7}$ was shown to contribute to $\widehat{\mathrm{CFD}}^{3}$ based on the choice of complex structure $J$. We now have

$$
m\left(w, \rho_{3}, \tau_{123}, \sigma_{3}, \sigma_{2}\right)=m\left(w, \rho_{3}, \tau_{3}\right)=s .
$$

Thus we have that $\left(R_{3} R_{4} R_{5} R_{6} R_{7}, \vec{\eta}_{2}\right)$ contributes to $\widehat{\mathrm{CFA}}^{3}$ or $\widehat{\mathrm{CFD}}^{3}$. Overall the domain $R_{3} R_{4} R_{5} R_{6} R_{7}$ contributes $\rho_{1} \tau_{123} \sigma_{12} s$ to $\partial(w)$.

$\boldsymbol{R}_{\mathbf{1}} \boldsymbol{R}_{\mathbf{2}} \boldsymbol{R}_{\mathbf{6}} \boldsymbol{R}_{\mathbf{7}} \boldsymbol{R}_{\mathbf{8}}$ This domain is compatible with $\vec{\eta}_{1}=\left(\rho_{2}, \rho_{3}, \tau_{1}, \sigma_{1}, \sigma_{2}, \sigma_{3}\right)$ and $\vec{\eta}_{2}=\left(\rho_{2}, \rho_{3}, \tau_{1}, \sigma_{123}\right)$. It has already been shown that there is no contribution with $\vec{\eta}_{1}$. For the contribution of $\vec{\eta}_{2}$, use the $\mathcal{A}_{\infty}$ relation for $\left(t, \rho_{2}, \rho_{1}, \sigma_{12}, \sigma_{3}\right)$. We look in Table 1 for domains starting at $t$ which involve $R_{6}$ and $R_{8}$ but not $R_{7}$, and some subset of $\left\{R_{1}, R_{2}\right\}$; there is only one option. The $\mathcal{A}_{\infty}$ relation becomes

$$
m\left(t, \rho_{2}, \rho_{1}, \mu\left(\sigma_{12}, \sigma_{3}\right)\right)=m\left(m\left(t, \rho_{2}, \sigma_{12}\right), \rho_{1}, \sigma_{3}\right) .
$$

The $\mathcal{A}_{\infty}$ relation for $\left(t, \rho_{2}, \sigma_{1}, \sigma_{2}\right)$ implies that $m\left(t, \rho_{2}, \sigma_{12}\right)=z$, so

$$
m\left(t, \rho_{2}, \rho_{1}, \sigma_{123}\right)=m\left(z, \rho_{1}, \sigma_{3}\right)=y \text {. }
$$

Thus by Lemma 2.8, the domain $R_{1} R_{2} R_{6} R_{7} R_{8}$ contributes $\rho_{23} \sigma_{123} y$ to $\partial(t)$.

$\boldsymbol{R}_{\mathbf{4}} \boldsymbol{R}_{\mathbf{5}} \boldsymbol{R}_{\mathbf{6}} \boldsymbol{R}_{\mathbf{7}} \boldsymbol{R}_{\mathbf{8}}$ This domain is compatible with $\vec{\eta}_{1}=\left(\tau_{2}, \tau_{3}, \sigma_{1}, \sigma_{2}, \sigma_{3}\right)$ and $\vec{\eta}_{2}=$ $\left(\tau_{2}, \tau_{3}, \sigma_{123}\right)$. With $\vec{\eta}_{1}$, the $R_{4} R_{5} R_{6} R_{7} R_{8}$ is realized as an immersed polygon, so the pair contributes to the differential. With $\vec{\eta}_{2}$, the contribution of this domain is determined by the $\mathcal{A}_{\infty}$ relation

$$
m\left(t, \tau_{2}, \tau_{1}, \mu\left(\sigma_{12}, \sigma_{3}\right)\right)=m\left(m\left(t, \tau_{2}, \sigma_{12}\right), \tau_{1}, \sigma_{3}\right)=m\left(x, \tau_{1}, \sigma_{3}\right)=y
$$

and Lemma 2.8. The domain contributes with $\vec{\eta}_{2}$, and the total mod 2 contribution of the domain is zero. 
$\boldsymbol{R}_{\mathbf{1}} \boldsymbol{R}_{\mathbf{2}} \boldsymbol{R}_{\mathbf{3}} \boldsymbol{R}_{\mathbf{4}} \boldsymbol{R}_{\mathbf{5}} \boldsymbol{R}_{\mathbf{6}} \boldsymbol{R}_{\mathbf{7}}$ This domain has four compatible sequences of Reeb chords:

$$
\begin{array}{cc}
\vec{\eta}_{1}=\left(\rho_{1}, \rho_{2}, \rho_{3}, \tau_{1}, \tau_{2}, \tau_{3}, \sigma_{1}, \sigma_{2}\right), & \vec{\eta}_{2}=\left(\rho_{1}, \rho_{2}, \rho_{3}, \tau_{123}, \sigma_{1}, \sigma_{2}\right) \\
\vec{\eta}_{3}=\left(\rho_{123}, \tau_{1}, \tau_{2}, \tau_{3}, \sigma_{1}, \sigma_{2}\right), & \vec{\eta}_{4}=\left(\rho_{123}, \tau_{123}, \sigma_{1}, \sigma_{2}\right)
\end{array}
$$

To obtain a boundary with Reeb chords $\vec{\eta}_{1}$, we must cut along all $\alpha$ arcs. This produces an immersed polygon, but there are too many edges and corners for Proposition 2.5 to apply. For instance, cutting along $\alpha_{1}^{\tau}$ produces corners at $h$, but the generators $v$ and $s$ do not contain the point $h$. Therefore the domain does not contribute with $\vec{\eta}_{1}$. For the other sequences of Reeb chords, we can use Lemma 2.8 and appropriate $\mathcal{A}_{\infty}$ relations. We find that the domain contributes with $\vec{\eta}_{2}$ and $\vec{\eta}_{4}$, and not with $\vec{\eta}_{3}$. Overall with $\mathbb{F}_{2}$ coefficients the contribution of this domain is zero.

\section{J Canceling differentials}

Putting everything together, the differential on $\widehat{\mathrm{CFD}}^{k}\left(\mathcal{Y}_{\mathcal{P}}\right)$ is recorded in Figure 13. The unlabeled arrow from $t$ to $s$ is the differential corresponding to the bigon $R_{9}$. This unlabeled edge can be canceled using the edge reduction algorithm for type $D$ structures described in Remark 2.1. We eliminate the arrow and the generators $t$ and $s$, and for every "zig-zag"

$$
\boldsymbol{x} \stackrel{a_{1}}{\longrightarrow} s \longleftarrow t \stackrel{a_{2}}{\longrightarrow} \boldsymbol{y},
$$

we introduce the new arrow $\boldsymbol{x} \stackrel{a_{1} \cdot a_{2}}{\longrightarrow} \boldsymbol{y}$. The resulting simplified form of $\widehat{\mathrm{CFD}}^{k}\left(\mathcal{Y}_{\mathcal{P}}\right)$ (which is quasi-isomorphic to the first diagram) is given in Figure 14.

\section{K Extremal spin ${ }^{c}$-structures}

To complete the computation of $\widehat{\mathrm{CFD}}^{3}\left(\mathcal{Y}_{\mathcal{P}}\right)$, we must compute $\widehat{\mathrm{CFD}}^{3}\left(\mathcal{Y}_{\mathcal{P}}, \mathfrak{s}\right)$ for other $\operatorname{spin}^{c}$-structures $\mathfrak{s}$.

$(1,2,0)$ Consider first the $\operatorname{spin}^{c}$-structure $\mathfrak{s}$ that has 1,2 and $0 \alpha$-arcs occupied on the $\rho, \sigma$ and $\tau$ boundaries, respectively. The only generator in this $\operatorname{spin}^{c}-$ structure is $a g i$, so $\widehat{\operatorname{CFD}}^{3}\left(\mathcal{Y}_{\mathcal{P}}, \mathfrak{s}\right)$ has one generator and no differentials.

$(2, \mathbf{1}, \mathbf{0})$ and $(2,0,1)$ The $\operatorname{spin}^{c}-$ structures with two arcs occupied on the $\rho$ boundary each have only one generator and no differentials. The generator with one $\alpha^{\sigma}$ arc is occupied is $a f i$, and the generator with one $\alpha^{\tau}$ arc occupied is $a f h$. 


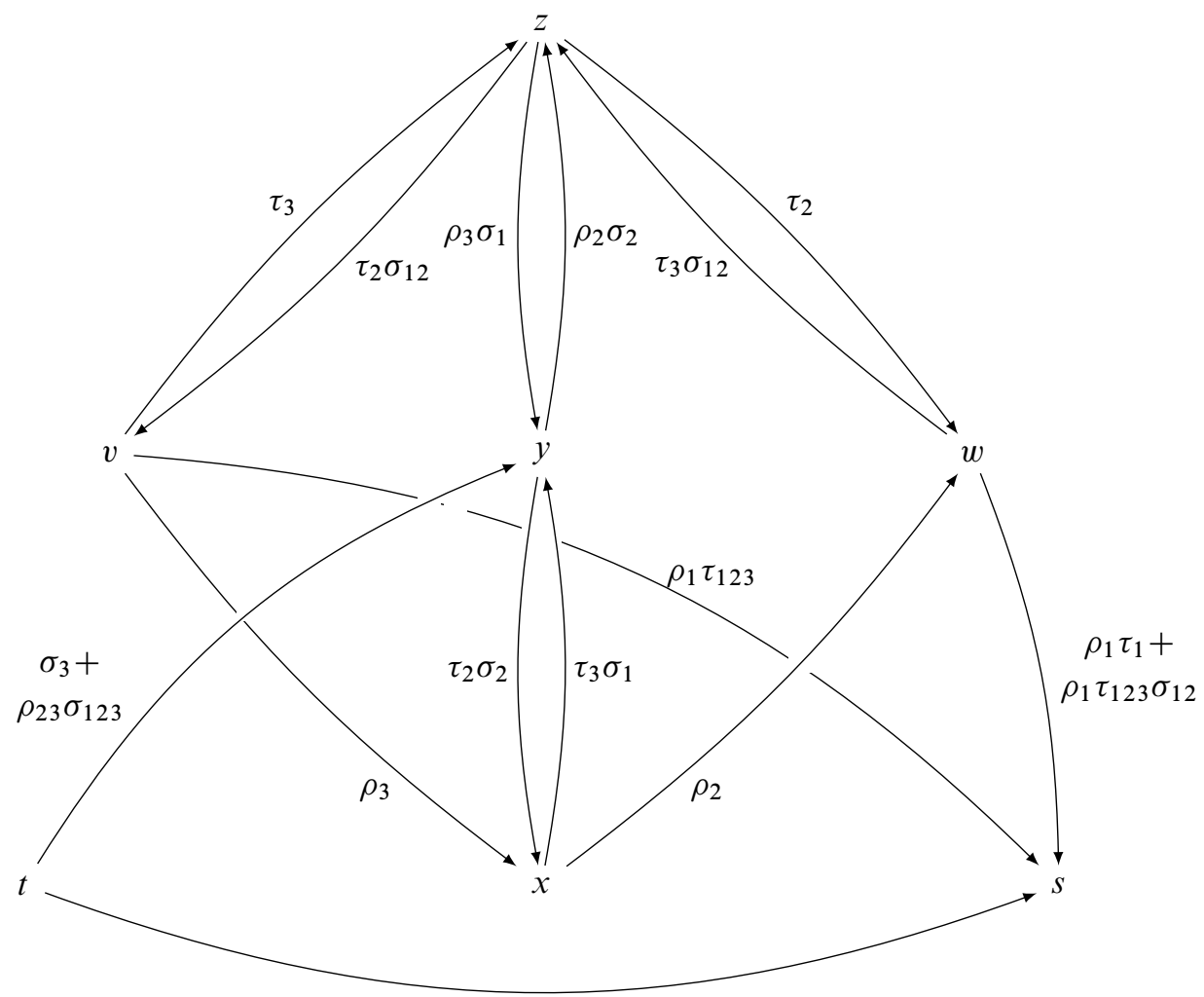

Figure 13: $\widehat{\mathrm{CFD}}^{k}\left(\mathcal{H}^{\prime}\right)$ in the middle spin ${ }^{c}$-structure, for the given choice of complex structure $J$

$\left(\mathbf{1 , 0 , 2 )}\right.$ The $\operatorname{spin}^{c}$-structure $\mathfrak{s}$ that has one $\alpha$ arc occupied on the $\rho$ boundary, zero on the $\sigma$ boundary and two on the $\tau$ boundary has 3 generators: aeh, $b f h$ and $d f h$. Recall that domains connecting these generators may not touch the $\sigma$ or $\tau$ boundaries, so we only need to consider the regions $R_{1}, R_{2}$ and $R_{9}$. It is easy to see that $R_{1}$ contributes a differential from $b f h$ to $a e h$ and $R_{2}$ contributes a differential from aeh to $d f h$ (they are both polygons). None of the generators include the points $i$ or $j$, so $R_{9}$ is not involved in any differentials. Finally, $R_{1} R_{2}$ does not contribute by Proposition 2.3; the corners make $R_{1} R_{2}$ a domain connecting $b f h$ to $d f h$, but neither of these generators contain a point on $\alpha_{1}^{\rho}$, which is required for a domain with Reeb chords $\left(\rho_{2}, \rho_{3}\right)$ to contribute nontrivially. For this $\operatorname{spin}^{c}$-structure, $\widehat{\operatorname{CFD}}^{3}\left(\mathcal{Y}_{\mathcal{P}}, \mathfrak{s}\right)$ is given by

$$
b f h \stackrel{\rho_{3}}{\longrightarrow} a e h \stackrel{\rho_{2}}{\longrightarrow} d f h .
$$




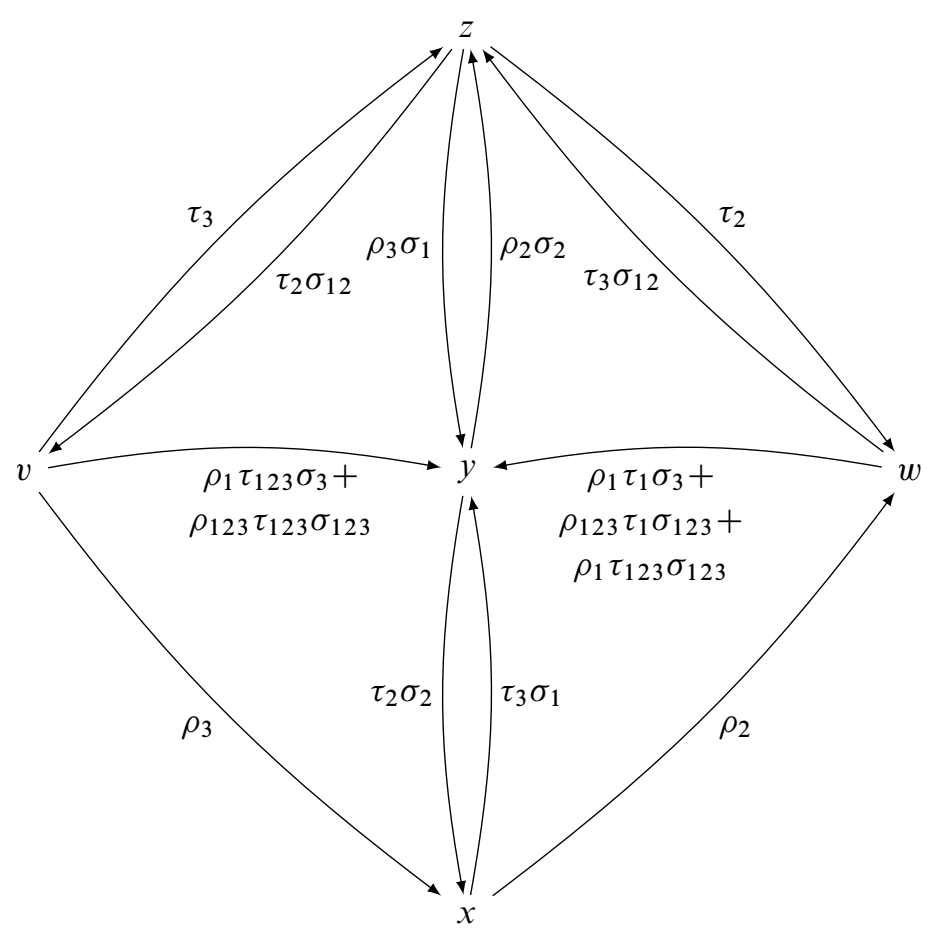

Figure 14: $\widehat{\mathrm{CFD}}^{3}\left(\mathcal{Y}_{\mathcal{P}}\right)$ in the middle spin ${ }^{c}$-structure after canceling the differential from $t$ to $s$ in $\widehat{\mathrm{CFD}}^{3}\left(\mathcal{H}^{\prime}\right)$

$(\mathbf{0 , 2}, \mathbf{1})$ This $\operatorname{spin}^{c}$-structure has 3 generators: $b g i, c g i$ and $d g i$. Domains that contribute to the differential do not touch the $\rho$ or $\sigma$ boundaries. The only domains which connect two generators are $R_{4}, R_{5}$ and $R_{4} R_{5}$. It is clear that the polygons $R_{4}$ and $R_{5}$ contribute, but the contribution of $R_{4} R_{5}$ is zero. Thus $\widehat{\operatorname{CFD}}^{3}\left(\mathcal{Y}_{\mathcal{P}}, \mathfrak{s}\right)$ for this $\operatorname{spin}^{c}$-structure is given by

$$
b g i \stackrel{\tau_{3}}{\longrightarrow} \operatorname{cg} i \stackrel{\tau_{2}}{\longrightarrow} d g i .
$$

$(\mathbf{0 , 1}, \mathbf{2})$ This $\operatorname{spin}^{c}-$ structure has 7 generators: $b g h, d g h, c e i, b j i, b k i, d j k$ and $d k i$. So $R_{9}$ contributes differentials from $b k i$ to $b j i$ and from $d k i$ to $d j i$. There can be no other differentials ending at $b j i$ or $d j i$, so the edge reduction algorithm lets us cancel these differentials and remove the generators $b k i, b j i, d k i$ and $d j i$ without adding new differentials (it is worth noting that we could also compute $\widehat{\operatorname{CFD}}^{3}\left(\mathcal{Y}_{\mathcal{P}}, \mathfrak{s}\right)$ using the Heegaard diagram $\mathcal{H}$ in Figure 7 instead of $\mathcal{H}^{\prime}$, and we would not have to deal with these four generators at all). The only domains connecting the remaining three generators are $R_{6}, R_{7}$ and $R_{6} R_{7}$. Once again, the individual regions contribute 
while $R_{6} R_{7}$ has zero contribution for idempotent reasons, and $\widehat{\mathrm{CFD}}^{3}\left(\mathcal{Y}_{\mathcal{P}}, \mathfrak{s}\right)$ is

$$
d g h \stackrel{\sigma_{2}}{\longrightarrow} c e i \stackrel{\sigma_{1}}{\longrightarrow} b g h .
$$

\section{L Gradings}

As described in Section $2 \mathrm{H}, \widehat{\mathrm{CFD}}^{3}\left(\mathcal{Y}_{\mathcal{P}}\right)$ is graded by a set which is a quotient of the noncommutative group $G_{3,0}$. We will compute this (relative) grading for the middle $\operatorname{spin}^{c}$-structure, using the form of $\widehat{\mathrm{CFD}}^{3}\left(\mathcal{Y}_{\mathcal{P}}\right)$ depicted in Figure 14.

We choose $x$ as the preferred generator and assign it the grading $\overrightarrow{0}=(0 ; 0,0 ; 0,0 ; 0,0)$. The arrow from $x$ to $w$ indicates that $\partial x$ contains the term $\rho_{2} w$, and thus $\operatorname{gr}(\partial x)=$ $\operatorname{gr}\left(\rho_{2} w\right)$. By the relation (7), we have

$$
\lambda^{-1} \operatorname{gr}(x)=\operatorname{gr}(\partial x)=\operatorname{gr}\left(\rho_{2}\right) \operatorname{gr}(w),
$$

and so

$$
\begin{aligned}
\operatorname{gr}(w) & =\lambda^{-1} \operatorname{gr}\left(\rho_{2}\right)^{-1} \operatorname{gr}(x)=\lambda^{-1}\left(\frac{1}{2} ;-\frac{1}{2},-\frac{1}{2} ; 0,0 ; 0,0\right) \overrightarrow{0} \\
& =\left(-\frac{1}{2} ;-\frac{1}{2},-\frac{1}{2} ; 0,0 ; 0,0\right) .
\end{aligned}
$$

Similarly, the arrow from $z$ to $w$ implies that $\lambda^{-1} \operatorname{gr}(z)=\operatorname{gr}(\partial z)=\operatorname{gr}\left(\tau_{2}\right) \operatorname{gr}(w)$, so

$$
\begin{aligned}
\operatorname{gr}(z) & =\lambda\left(-\frac{1}{2} ; 0,0 ; 0,0 ; \frac{1}{2}, \frac{1}{2}\right)\left(-\frac{1}{2} ;-\frac{1}{2},-\frac{1}{2} ; 0,0 ; 0,0\right) \\
& =\left(0 ;-\frac{1}{2},-\frac{1}{2} ; 0,0 ; \frac{1}{2}, \frac{1}{2}\right) .
\end{aligned}
$$

The arrow from $v$ to $z$ implies that

$$
\begin{aligned}
\operatorname{gr}(v) & =\lambda \operatorname{gr}\left(\tau_{3}\right) \operatorname{gr}(z) \\
& =\lambda\left(-\frac{1}{2} ; 0,0 ; 0,0 ;-\frac{1}{2}, \frac{1}{2}\right)\left(0 ;-\frac{1}{2},-\frac{1}{2} ; 0,0 ; \frac{1}{2}, \frac{1}{2}\right) \\
& =\left(0 ;-\frac{1}{2},-\frac{1}{2} ; 0,0 ; 0,1\right) .
\end{aligned}
$$

Finally, the arrow from $y$ to $x$ implies that

$$
\begin{aligned}
\operatorname{gr}(y) & =\lambda \operatorname{gr}\left(\sigma_{2}\right) \operatorname{gr}\left(\tau_{2}\right) \operatorname{gr}(x) \\
& =\lambda\left(-\frac{1}{2} ; 0,0 ; \frac{1}{2}, \frac{1}{2} ; 0,0\right)\left(-\frac{1}{2} ; 0,0 ; 0,0 ; \frac{1}{2}, \frac{1}{2}\right) \overrightarrow{0} \\
& =\left(0 ; 0,0 ; \frac{1}{2}, \frac{1}{2} ; \frac{1}{2}, \frac{1}{2}\right) .
\end{aligned}
$$

We have now computed the gradings of each generator as elements of the group $G_{3,0}$. However, these gradings are only well defined modulo the action of $\mathcal{P}(x)$, the group generated by the gradings of periodic domains connecting $x$ to itself. To finish the computation, we need to find $\mathcal{P}(x)$. 
Consider the arrow from $v$ to $x$, which implies that $\lambda^{-1} \operatorname{gr}(v)=\operatorname{gr}\left(\rho_{3}\right) \operatorname{gr}(x)$. It follows that

$$
\begin{aligned}
\operatorname{gr}(x) & =\lambda^{-1} \operatorname{gr}\left(\rho_{3}\right)^{-1} \operatorname{gr}(v) \\
& =\lambda^{-1}\left(\frac{1}{2} ; \frac{1}{2},-\frac{1}{2} ; 0,0 ; 0,0\right)\left(0 ;-\frac{1}{2},-\frac{1}{2} ; 0,0 ; 0,1\right) \\
& =(-1 ; 0,-1 ; 0,1 ; 0,0) .
\end{aligned}
$$

We have that $\operatorname{gr}(x)=(-1 ; 0,-1 ; 0,1 ; 0,0)$, but also that $\operatorname{gr}(x)=\overrightarrow{0}$. It follows that $(-1 ; 0,-1 ; 0,1 ; 0,0)$ and $\overrightarrow{0}$ are equivalent modulo $\mathcal{P}(x)$, and thus we have $(-1 ; 0,-1 ; 0,1 ; 0,0) \in \mathcal{P}(x)$. In fact, since this nonzero value of $\operatorname{gr}(x)$ was obtained from $\overrightarrow{0}$ by following a loop of edges with oriented labels $\left(\rho_{2},-\tau_{2},-\tau_{3}, \rho_{3}\right)$, the difference $(-1 ; 0,-1 ; 0,1 ; 0,0)$ corresponds to the grading of a periodic domain with boundary $\rho_{23}-\tau_{23}$.

Another value for $\operatorname{gr}(x)$, and thus another element of $\mathcal{P}(x)$, can be found by considering the arrow from $x$ to $y$. We have that

$$
\begin{aligned}
\operatorname{gr}(x) & =\lambda \operatorname{gr}\left(\sigma_{1}\right) \operatorname{gr}\left(\tau_{3}\right) \operatorname{gr}(y) \\
& =\lambda\left(-\frac{1}{2} ; 0,0 ; \frac{1}{2},-\frac{1}{2} ; 0,0\right)\left(-\frac{1}{2} ; 0,0 ; 0,0 ;-\frac{1}{2}, \frac{1}{2}\right)\left(0 ; 0,0 ; \frac{1}{2}, \frac{1}{2} ; \frac{1}{2}, \frac{1}{2}\right) \\
& =(0 ; 0,0 ; 1,0 ; 0,1) .
\end{aligned}
$$

So $(0 ; 0,0 ; 1,0 ; 0,1)$ is an element of $\mathcal{P}(x)$, corresponding to a periodic domain with boundary $\sigma_{12}+\tau_{23}$.

Consider the loop formed by the arrow from $y$ to $x$, the $\rho_{1} \sigma_{3} \tau_{123}$ arrow from $w$ to $x$ and the arrow from $x$ to $w$. This loop corresponds to a periodic domain with boundary $\rho_{12}+\sigma_{23}+\tau_{12}$. As before, starting with $\operatorname{gr}(x)=\overrightarrow{0}$ the arrow from $y$ to $x$ implies that $\operatorname{gr}(y)=\left(0 ; 0,0 ; \frac{1}{2}, \frac{1}{2} ; \frac{1}{2}, \frac{1}{2}\right)$. The arrow from $w$ to $y$ then implies that

$$
\begin{aligned}
\operatorname{gr}(w) & =\lambda \operatorname{gr}\left(\rho_{1}\right) \operatorname{gr}\left(\sigma_{3}\right) \operatorname{gr}\left(\tau_{1}\right) \operatorname{gr}(y) \\
& =\lambda\left(-\frac{1}{2} ; \frac{1}{2},-\frac{1}{2} ; 0,0 ; 0,0\right)\left(-\frac{1}{2} ; 0,0 ;-\frac{1}{2}, \frac{1}{2} ; 0,0\right)\left(-\frac{1}{2} ; 0,0 ; 0,0 ; \frac{1}{2},-\frac{1}{2}\right) \operatorname{gr}(y) \\
& =\left(-\frac{1}{2} ; \frac{1}{2},-\frac{1}{2} ;-\frac{1}{2}, \frac{1}{2} ; \frac{1}{2},-\frac{1}{2}\right)\left(0 ; 0,0 ; \frac{1}{2}, \frac{1}{2} ; \frac{1}{2}, \frac{1}{2}\right) \\
& =\left(-\frac{1}{2} ; \frac{1}{2},-\frac{1}{2} ; 0,1 ; 1,0\right) .
\end{aligned}
$$

The arrow from $x$ to $w$ then implies that

$$
\begin{aligned}
\operatorname{gr}(x) & =\lambda \operatorname{gr}\left(\rho_{2}\right) \operatorname{gr}(w) \\
& =\lambda\left(-\frac{1}{2} ; \frac{1}{2}, \frac{1}{2} ; 0,0 ; 0,0\right)\left(-\frac{1}{2} ; \frac{1}{2},-\frac{1}{2} ; 0,1 ; 1,0\right) \\
& =\left(-\frac{1}{2} ; 1,0 ; 0,1 ; 1,0\right)
\end{aligned}
$$

is an element of $\mathcal{P}(x)$. 
Since $\mathcal{Y}_{\mathcal{P}}$ has 3 boundary components, the space of periodic domains has dimension 3 . Since the three elements of $\mathcal{P}(x)$ we have found are independent, they are enough to determine $\mathcal{P}(x)$ :

$$
\mathcal{P}(x)=\left\langle(-1 ; 0,-1 ; 0,1 ; 0,0),(0 ; 0,0 ; 1,0 ; 0,1),\left(-\frac{1}{2} ; 1,0 ; 0,1 ; 1,0\right)\right\rangle .
$$

\section{Self gluing}

Any graph manifold which is represented by a tree with only genus zero vertices can be obtained by gluing together copies of $\mathcal{Y}_{\mathcal{P}}$, solid tori, and mapping cylinders of appropriate Dehn twists. Each time a new piece is glued on, the new bordered Heegaard Floer invariants can be obtained as a box tensor product by the pairing theorem.

To build up an arbitrary graph manifold from these building blocks, however, it is often necessary to glue two boundaries of a 3-manifold together. The resulting bordered invariants are obtained by taking Hochschild homology, but we must first tensor with an additional bimodule which corresponds to the bordered Heegaard diagram $\mathcal{H}_{\mathrm{SG}}$ in Figure 15.

The process of self-gluing is discussed in [11, Section 4.4], and a Heegaard diagram isotopic to $\mathcal{H}_{\mathrm{SG}}$ is given there. We recall here the topological interpretation of $\mathcal{H}_{\mathrm{SG}}$. Adding 2-handles along the curves $\beta_{1}$ and $\beta_{2}$ produces the manifold $F \times[0,1]$, where $F$ is the twice punctured torus, that is, $T^{2} \times[0,1]$ with two tubes drilled out. The curves $\alpha_{0}$ and $\beta_{0}$ correspond to meridians of these tubes, so adding 2-handles along $\alpha_{0}$ and $\beta_{0}$ has the effect of filling in these tubes with meridional disks (for this reason, $\mathcal{H}_{\mathrm{SG}}$ is referred to as the tube-cutting piece in [11]).

The following lemma captures the importance of $\mathcal{H}_{\mathrm{SG}}$ to self-gluing (the two boundary case of this lemma is implicit in the proof of Theorem 12 in [11]):

Lemma 4.1 Let $\mathcal{H}$ be a bordered Heegaard diagram for a bordered 3-manifold $Y$ with at least two (toroidal) boundary components. Let $\mathcal{H}^{\prime}$ be obtained from $\mathcal{H}$ by gluing the first and second boundary components of $\mathcal{H}$ to each other with a copy of $\mathcal{H}_{\mathrm{SG}}$ in between, and let $Y^{\prime}$ be obtained from $Y$ by gluing the first and second boundary components together. Then $\mathcal{H}^{\prime}$ is a (bordered) Heegaard diagram for $Y^{\prime}$.

Proof Recall that a 3-manifold is obtained from a (bordered) Heegaard diagram by attaching 2-handles to the thickened Heegaard surface along $\alpha$ curves on one side and along $\beta$ curves on the other side. In the closed case, the result has an $S^{2}$ boundary component on each side, which we cap off with 3-balls. In the bordered case, we also 


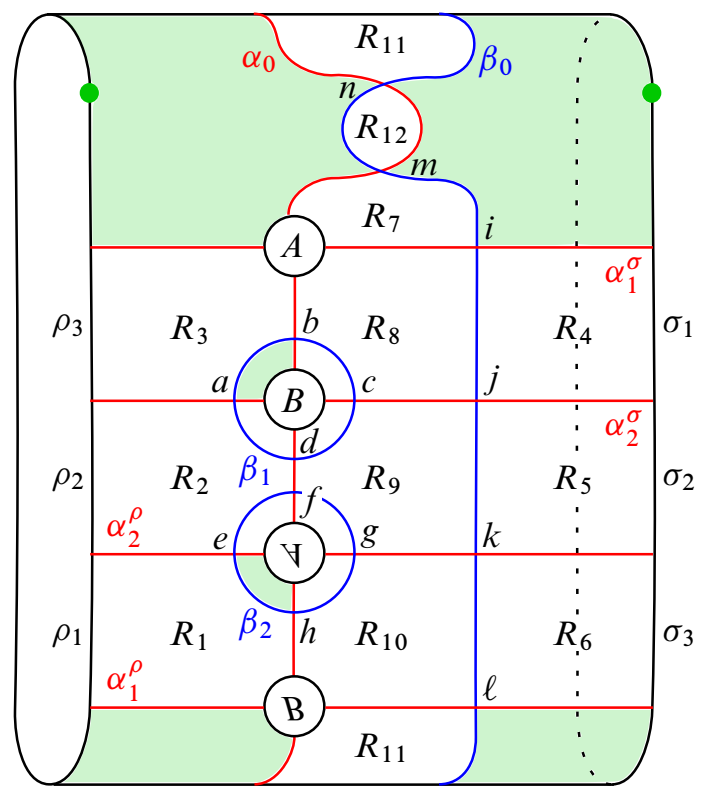

Figure 15: A Heegaard diagram $\mathcal{H}_{\mathrm{SG}}$ for the self gluing bimodule

complete the manifold by adding a 3-ball on each side, but each 3-ball intersects each boundary component of the resulting bordered 3-manifold in a disk.

Let $H$ be the 3-manifold obtained by adding 2-handles to $\mathcal{H}$ as above, so that $Y=H \cup B_{1} \cup B_{2}$, where $B_{1}$ and $B_{2}$ are 3-balls. Note that $Y^{\prime}=H^{\prime} \cup B_{1}^{\prime} \cup B_{2}^{\prime}$, where $H^{\prime}$ is obtained from $\mathcal{H}$ by gluing the appropriate two boundary components and adding 2-handles as before, and $B_{i}^{\prime}$ is obtained from $B_{i}$ by identifying two disks on the boundary. Thus $Y^{\prime}$ is obtained from $H^{\prime}$ by filling in two tubes with solid tori. This can be accomplished by adding a meridional disk followed by a 3-ball for each solid torus. Inserting $\mathcal{H}_{\mathrm{SG}}$ between the two boundaries of $\mathcal{H}$ has exactly the effect of adding these meridional disks. That is, attaching 2-handles to $\mathcal{H}^{\prime}$ is the same as attaching 2-handles to $\mathcal{H}$ with the relevant boundaries glued (which produces $H^{\prime}$ ) and then attaching a 2-handle to a meridional curve on each "tube". Adding a 3-ball to each side then produces $Y^{\prime}$, and so $\mathcal{H}^{\prime}$ is a Heegaard diagram for $Y^{\prime}$.

Note that $\mathcal{H}_{\mathrm{SG}}$ is not a valid bordered Heegaard diagram because it has too many $\alpha$ and $\beta$ curves; nevertheless we can associate a bimodule to it. Strictly speaking, $\mathcal{H}_{\mathrm{SG}}$ is a bordered-sutured Heegaard diagram, as defined in [32], and the bimodule is the bordered-sutured Floer bimodule $\widehat{\operatorname{BSDD}}\left(\mathcal{H}_{\mathrm{SG}}\right)$. However, since it does not affect the computation of the invariant we will not emphasize this distinction, and by abuse of notation we refer to this bimodule as $\widehat{\mathrm{CFDD}}\left(\mathcal{H}_{\mathrm{SG}}\right)$. 
The type $A$ version of this bimodule lets us compute invariants of self-glued manifolds:

Proposition 4.2 Let $Y$ be the bordered 3-manifold $Y$ with $k \geq 2$ toroidal boundary components. Then

$$
\mathrm{HH}_{*}\left(\widehat{\mathrm{CFD}}^{k}(Y) \otimes \widehat{\mathrm{CFAA}}\left(\mathcal{H}_{\mathrm{SG}}\right)\right) \cong \widehat{\mathrm{CFD}}^{k-2}\left(Y^{\prime}\right),
$$

where $Y^{\prime}$ is the manifold obtained from $Y$ by gluing the two appropriate boundary components.

Proof This is a straightforward generalization of Theorem 12 in [11], which considers the case that $Y$ has only two boundary components. We will not repeat the proof, but the essential observation is contained in Lemma 4.1.

The focus of the present section is to compute the invariant $\widehat{\mathrm{CFDD}}\left(\mathcal{H}_{\mathrm{SG}}\right)$. We first restrict our attention to the middle $\operatorname{spin}^{c}$-structure, where exactly one $\alpha$ arc is occupied at each boundary component. In fact, this computation gives all of $\widehat{\operatorname{CFDD}}\left(\mathcal{H}_{\mathrm{SG}}\right)$; it will be shown at the end of this section that the other summands for $\widehat{\mathrm{CFDD}}\left(\mathcal{H}_{\mathrm{SG}}\right)$ are trivial.

Complex structure As always, the computation of $\widehat{\mathrm{CFDD}}\left(\mathcal{H}_{\mathrm{SG}}\right)$ depends on the complex structure $J$ chosen for the Heegaard surface $\Sigma$. We will make the following assumptions:

- $\Theta_{8,9}^{d, b}<\Theta_{8,9}^{i, k}$

- $\Theta_{9,10}^{h, f}<\Theta_{9,10}^{j, l}$;

- $\Theta_{2,3,8,9}^{a, a}<\Theta_{2,3,8,9}^{i, k}$;

- $\Theta_{1,2,9,10}^{e, e}<\Theta_{1,2,9,10}^{j, l}$.

We also assume that the following arcs are sufficiently pinched to apply Proposition 2.7 when necessary:

- an arc $\gamma_{1}$ through $R_{1}, R_{2}$ and $R_{3}$ parallel to $\alpha_{0}$, connecting $\beta_{2}$ in $R_{1}$ to $\beta_{1}$ in $R_{3}$;

- an arc $\gamma_{2}$ in $R_{7}$ parallel to $\alpha_{0}$, connecting $\beta_{0}$ to $\beta_{2}$;

- an arc $\gamma_{3}$ in $R_{11}$ parallel to $\alpha_{0}$, connecting $\beta_{0}$ to $\beta_{1}$.

Finally, we will also assume that $\Theta_{2,5,8,9,9,10}^{h, b}<\Theta_{2,5,8,9,9,10}^{i, \ell}$, where here $\Theta_{2,5,8,9,9,10}$ refers to the appropriate ratio of $\alpha$ and $\beta$ lengths for the annulus obtained from $R_{2} R_{5} R_{8} R_{9} R_{9} R_{10}$ by pinching along the arc through $R_{2}$ mentioned above; see Figure 16. 


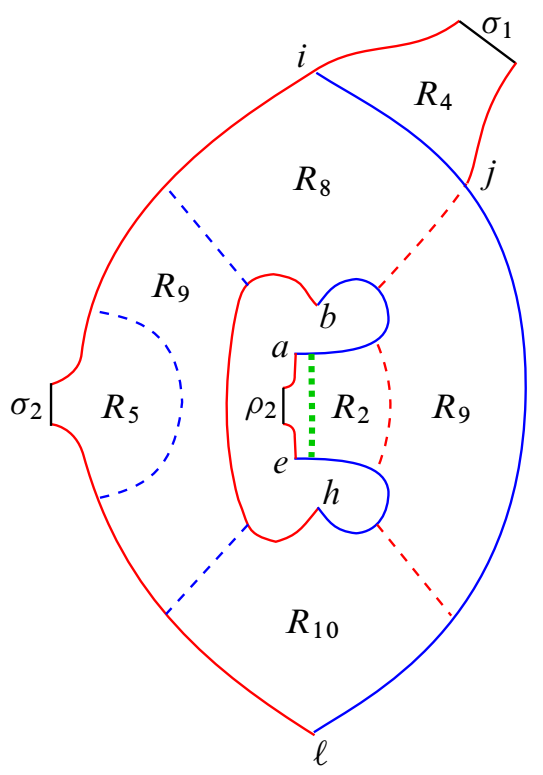

Figure 16: The domain $R_{2} R_{4} R_{5} R_{8} R_{9} R_{9} R_{10}$. It is just like the annulus $A+B_{2}$ in Figure 2, except that the inner boundary has too many $\alpha$ and $\beta$ segments. To fix this, we pinch along the green dotted arc in $R_{2}$.

To check that these assumptions can be made consistently, note that for all five of the annuli in question, the boundary component for which the ratio $\Theta$ of $\alpha$ length to $\beta$ length is assumed to be large lies on $\beta_{0}$ between $i$ and $\ell$ and $\alpha_{1}^{\sigma} \cup \alpha_{2}^{\sigma}$. In each case, the boundary component for which $\Theta$ is assumed to be small lies on $\alpha_{0} \cup \alpha_{1}^{\rho} \cup \alpha_{2}^{\rho}$ and $\beta_{1} \cup \beta_{2}$. The assumptions concerning these annuli can be satisfied by assuming that $\alpha_{0}, \alpha_{1}^{\rho}, \alpha_{2}^{\rho}$ and $\beta_{0}$ between $i$ and $\ell$ are all very short (say by pinching along arcs parallel to these curves) and other $\alpha$ and $\beta$ curves are relatively long. Finally, note that the three arcs $\gamma_{1}, \gamma_{2}$ and $\gamma_{3}$ do not intersect any of the segments that are assumed to be short, so these arcs can be pinched without affecting the annuli.

Generators $\widehat{\mathrm{CFDD}}\left(\mathcal{H}_{\mathrm{SG}}\right)$ has 20 generators in the middle spin ${ }^{c}$-structure:

$\begin{array}{lllllllll}\text { afi } & \text { afj } & \text { afk afl ahi ahj ahk ahl ang amg } \\ \text { ebi ebj ebk ebl edi edj edk edl enc emc }\end{array}$

Domains To list the domains that might contribute to $\widehat{\mathrm{CFDD}}\left(\mathcal{H}_{\mathrm{SG}}\right)$, note that the multiplicities of the regions on the boundary $\left(R_{1}, \ldots, R_{6}\right)$ are at most 1 , and the region $R_{12}$ cannot be combined with any other regions. Checking all positive connected domains which satisfy these conditions for appropriate corner multiplicity, we find 292 


\begin{tabular}{|c|c|c|c|c|c|c|c|c|}
\hline Regions & $x$ & $y$ & Regions & $x$ & $y$ & Regions & $x$ & $y$ \\
\hline 1 & $e d i$ & $a h i$ & $1,2,3$ & $e b l$ & $a h l$ & $2,3,5,8,9$ & $a h i$ & $a h j$ \\
\hline 1 & $e d j$ & $a h j$ & $1,6,10$ & $e n c$ & ang & $2,3,7,8,9$ & $a m g$ & $a h k$ \\
\hline 1 & $e d k$ & $a h k$ & $1,6,10$ & emc & $a m g$ & $2,4,5,8,9$ & afl & $e b l$ \\
\hline 1 & $e d l$ & $a h l$ & $1,9,10$ & $e d j$ & afl & $2,5,6,9,10$ & $a h i$ & $e d i$ \\
\hline 2 & $a f i$ & $e d i$ & $1,10,11$ & $e b k$ & ang & $3,4,5,8,9$ & $e d l$ & afl \\
\hline 2 & $a f j$ & $e d j$ & $2,5,9$ & ang & $e n c$ & $3,7,8,9,10$ & $e m c$ & afl \\
\hline 2 & $a f k$ & $e d k$ & $2,5,9$ & $a m g$ & $e m c$ & $4,5,6,8,9$ & $e d l$ & $e b k$ \\
\hline 2 & $a f l$ & $e d l$ & $2,8,9$ & $a f i$ & $e b k$ & $4,5,6,9,10$ & $a h j$ & $a f i$ \\
\hline 3 & $e b i$ & $a f i$ & $2,9,10$ & $a h j$ & edl & $4,5,8,9,11$ & $e d l$ & enc \\
\hline 3 & $e b j$ & $a f j$ & $3,4,8$ & $e n c$ & ang & $4,8,9,10,11$ & $a h j$ & ang \\
\hline 3 & $e b k$ & $a f k$ & $3,4,8$ & $e m c$ & $a m g$ & $5,6,7,9,10$ & $a m g$ & $a f i$ \\
\hline 3 & $e b l$ & afl & $3,7,8$ & emc & $a h j$ & $6,7,8,9,10$ & $e m c$ & $e b k$ \\
\hline 4 & $a f j$ & $a f i$ & $3,8,9$ & $e d i$ & afk & $7,8,9,10,11$ & $a m g$ & ang \\
\hline 4 & $a h j$ & $a h i$ & $4,5,6$ & $a f l$ & afi & $7,8,9,10,11$ & $e m c$ & $e n c$ \\
\hline 4 & $e b j$ & $e b i$ & $4,5,6$ & $a h l$ & $a h i$ & $1,2,3,4,5,8,9$ & $e d l$ & $a h l$ \\
\hline 4 & $e d j$ & $e d i$ & $4,5,6$ & $e b l$ & $e b i$ & $1,2,3,5,6,9,10$ & $e b i$ & afi $i$ \\
\hline 5 & $a f k$ & $a f j$ & $4,5,6$ & $e d l$ & $e d i$ & $1,2,3,7,8,9,10$ & $e m c$ & $a h l$ \\
\hline 5 & $a h k$ & $a h j$ & $4,8,9$ & $e d j$ & $e b k$ & $1,2,3,8,9,10,11$ & $e b i$ & ang \\
\hline 5 & $e b k$ & $e b j$ & $4,8,11$ & afl & ang & $1,2,4,5,6,9,10$ & $e b j$ & $e b i$ \\
\hline 5 & $e d k$ & $e d j$ & $5,8,9$ & $e d i$ & $e b j$ & $1,2,5,8,9,9,10$ & $e d i$ & $e b l$ \\
\hline 6 & afl & $a f k$ & $5,9,10$ & $a h k$ & afl & $2,3,4,5,6,8,9$ & $a h l$ & $a h k$ \\
\hline 6 & $a h l$ & $a h k$ & $6,7,10$ & emc & $e d i$ & $2,3,5,8,9,9,10$ & $a h i$ & afl \\
\hline 6 & $e b l$ & $e b k$ & $6,9,10$ & $a h j$ & $a f k$ & $2,4,5,8,9,9,10$ & $a h j$ & $e b l$ \\
\hline 6 & $e d l$ & $e d k$ & $1,2,3,8,9$ & edi & $a h k$ & $2,5,6,8,9,9,10$ & $a h i$ & $e b k$ \\
\hline 7 & amg & $a h i$ & $1,2,3,9,10$ & $e b j$ & afl & $2,5,7,8,9,9,10$ & $a m g$ & $e b l$ \\
\hline 11 & $e b l$ & $e n c$ & $1,2,5,9,10$ & $e b k$ & $e b l$ & $2,5,8,9,9,10,11$ & $a h i$ & enc \\
\hline 12 & $a m g$ & ang & $1,2,6,9,10$ & $e b j$ & $e b k$ & $4,5,6,7,8,9,10$ & $e m c$ & $e b i$ \\
\hline 12 & emc & $e n c$ & $1,2,9,10,11$ & $e b j$ & $e n c$ & $4,5,6,8,9,10,11$ & $a h l$ & ang \\
\hline $1,2,3$ & $e b i$ & $a h i$ & $1,5,6,9,10$ & $e d i$ & afi & $1,2,3,4,5,6,8,9,10$ & $e n c$ & ang \\
\hline $1,2,3$ & $e b j$ & $a h j$ & $1,8,9,10,11$ & $e d i$ & ang & $1,2,3,4,5,6,8,9,10$ & emc & $a m g$ \\
\hline $1,2,3$ & $e b k$ & ahk & $2,3,4,8,9$ & $a h j$ & $a h k$ & & & \\
\hline
\end{tabular}

Table 4: List of 92 domains that might contribute to $\widehat{\mathrm{CFDD}}\left(\mathcal{H}_{\mathrm{SG}}\right)$, with the corresponding initial generators $\boldsymbol{x}$ and final generators $\boldsymbol{y}$

domains to consider. Of these, 200 can be eliminated by Lemma 2.4 or Proposition 2.3 (though we should make note of these domains, in case they come up when checking $\mathcal{A}_{\infty}$ relations). All of these steps are easy to perform with a computer program. The remaining 92 domains are listed in Table 4. 

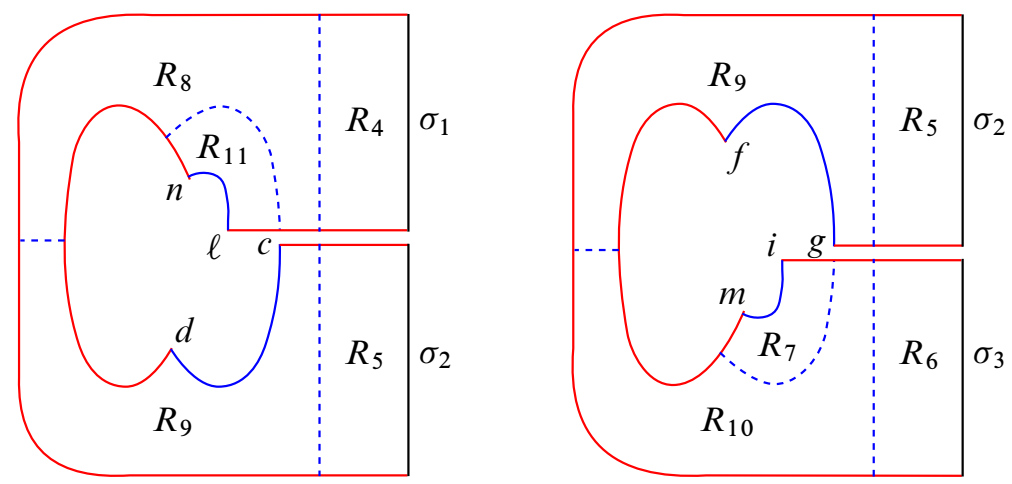

Figure 17: Regions $R_{4} R_{5} R_{8} R_{9} R_{11}$ and $R_{5} R_{6} R_{7} R_{9} R_{10}$ are both realized as immersed polygons when cut along $\alpha_{2}^{\sigma}$ and $\alpha_{1}^{\sigma}$, respectively.

Polygons All of the single region domains are easily seen to be polygons, and thus contribute to the differential by Proposition 2.5. In addition, it is easy to check that the following domains are polygons: $R_{1} R_{6} R_{10}, R_{1} R_{10} R_{11}, R_{2} R_{5} R_{9}, R_{3} R_{4} R_{8}$, $R_{3} R_{7} R_{8}, R_{4} R_{8} R_{11}$ and $R_{6} R_{7} R_{10}$. Each domain has only one sequence of Reeb chords to consider. Thus by Proposition 2.5 each of these domains contributes, and we have quickly dispatched 38 of the entries in Table 4.

The domain $R_{4} R_{5} R_{8} R_{9} R_{11}$ is a polygon, though it may not be obvious at first glance. The only compatible sequence of Reeb chords is $\left(\sigma_{1}, \sigma_{2}\right)$. To realize the domain as an immersed surface with boundary Reeb chords $\left(\sigma_{1}, \sigma_{2}\right)$, we must cut along $\alpha_{2}^{\sigma}$, which produces a polygon; see Figure 17. Similarly, the domain $R_{5} R_{6} R_{7} R_{9} R_{10}$ corresponds to a polygon with boundary $\left(\sigma_{2}, \sigma_{3}\right)$ after cutting along $\alpha_{1}^{\sigma}$. By Proposition 2.5, both of these domains contribute to the differential.

$R_{1} R_{2} R_{3}$ and $R_{4} R_{5} R_{6}$ can also be realized as polygons, and thus contribute with Reeb chords $\left(\rho_{1}, \rho_{2}, \rho_{3}\right)$ and $\left(\sigma_{123}\right)$, respectively. Furthermore, $R_{4} R_{5} R_{6}$ can not contribute with its other sequence of compatible Reeb chords, $\left(\sigma_{1}, \sigma_{2}, \sigma_{3}\right)$, since cutting along the $\alpha^{\sigma}$ arcs from the boundary would produce a disconnected domain. Overall the domain $R_{4} R_{5} R_{6}$ contributes to the differential for each pair of generators it connects. The contribution of $R_{1} R_{2} R_{3}$ with $\left(\rho_{123}\right)$ can be understood by examining the $\mathcal{A}_{\infty}$ relations for $\left(e b *, \rho_{1}, \rho_{23}\right)$ and $\left(a f *, \rho_{2}, \rho_{3}\right)$, where $*$ can be $i, j, k$ or $l$. The relations imply that

$$
m\left(e b *, \rho_{123}\right)=m\left(m\left(m\left(e b *, \rho_{1}\right), \rho_{2}\right), \rho_{3}\right)=a h * .
$$

Since the operation is nontrivial in $\widehat{\mathrm{CFAA}}$, we have that $\left(R_{1} R_{2} R_{3},\left(\rho_{123}\right)\right)$ contributes to the differential from $e b *$ to $a h *$ in $\widehat{\mathrm{CFDD}}\left(\mathcal{H}_{\mathrm{SG}}\right)$ by Lemma 2.8 , and the total mod 2 contribution of $R_{1} R_{2} R_{3}$ is zero. 
Simple annuli $R_{8} R_{9}$ is an index-zero annulus analogous to $A$ in Proposition 2.6. The four domains obtained by adding $R_{2}, R_{3}, R_{4}$ or $R_{5}$ to this annulus may or may not contribute to $\widehat{\mathrm{CFDD}}\left(\mathcal{H}_{\mathrm{SG}}\right)$, depending on the choice of complex structure on $R_{8} R_{9}$. Since we have chosen $J$ such that $\Theta_{8,9}^{d, b}<\Theta_{8,9}^{i, k}$, Proposition 2.6 asserts that none of these four domains contributes. Notice that since $R_{2}$ and $R_{3}$ are not bigons, we must first use Proposition 2.7 to pinch off the extra $\alpha$ portion of the boundary, and then we can apply Proposition 2.6. We specifically chose the complex structure $J$ to be consistent with pinching the appropriate $\operatorname{arcs}$ in $R_{2}$ and $R_{3}$.

Similarly, $R_{9} R_{10}$ is an index-zero annulus to which the regions $R_{1}, R_{2}, R_{5}$ or $R_{6}$ may be added. Given the choice that $\Theta_{9,10}^{h, f}<\Theta_{9,10}^{j, l}$, none of the four corresponding domains contribute.

Two more direct applications of Proposition 2.6 involve the annuli $R_{2} R_{3} R_{8} R_{9}$ and $R_{1} R_{2} R_{9} R_{10}$. Given that $\Theta_{2,3,8,9}^{a, a}<\Theta_{2,3,8,9}^{i, k}$, we have that $R_{2} R_{3} R_{7} R_{8} R_{9}$ contributes to $\widehat{\mathrm{CFDD}}\left(\mathcal{H}_{\mathrm{SG}}\right)$, but $R_{2} R_{3} R_{4} R_{8} R_{9}$ and $R_{2} R_{3} R_{5} R_{8} R_{9}$ do not. The fact that $\Theta_{1,2,9,10}^{e, e}<\Theta_{1,2,9,10}^{j, l}$ implies that $R_{1} R_{2} R_{9} R_{10} R_{11}$ contributes to $\widehat{\operatorname{CFDD}}\left(\mathcal{H}_{\mathrm{SG}}\right)$, but $R_{1} R_{2} R_{5} R_{9} R_{10}$ and $R_{1} R_{2} R_{6} R_{9} R_{10}$ do not. Note that for $R_{2} R_{3} R_{7} R_{8} R_{9}$ and $R_{1} R_{2} R_{9} R_{10} R_{11}$ we make use of Proposition 2.7 and the relevant assumptions about the complex structure on $R_{7}$ and $R_{11}$.

Finally, we will use Proposition 2.6 to account for the domain $R_{2} R_{4} R_{5} R_{8} R_{9} R_{9} R_{10}$, which connects $a h j$ to $e b l$. There is only one way to piece together these regions so that there are no unwanted corners, which is shown in Figure 16. If we pinch $R_{2}$ along the arc connecting the two $\beta$ curves, then this domain has exactly the form of $D_{2}$ in Proposition 2.6. Since we chose $J$ such that $\Theta_{2,5,8,9,9,10}^{h, b}<\Theta_{2,5,8,9,9,10}^{i, \ell}$, it follows that this domain does not count.

More annuli The domain $R_{1} R_{2} R_{3} R_{8} R_{9}$, with the Reeb chords $\left(\rho_{1}, \rho_{2}, \rho_{3}\right)$, is an annulus with one obtuse corner. If we pinch along the arc through $R_{1}, R_{2}$ and $R_{3}$ parallel to $\alpha_{0}$, the modified annulus has one $\alpha$ and one $\beta$ segment on each boundary component. In this situation, we can apply the same reasoning as the proof of Proposition 2.6 (the only difference is that the cuts from the obtuse corner do not leave the annulus through to the opposite boundary component). Cutting along $\beta_{1}$ from $d$ makes the length of $\beta$ on the boundary component containing $d$ grow, so that $\theta_{1,2,3,8,9}^{d, a}(c)$ approaches $-\infty$ as $c$ approaches $+\infty$, and $\theta_{1,2,3,8,9}^{d, a}(c)-\theta_{1,2,3,8,9}^{i, k}(c)$ is negative. On the other hand, cutting along $\alpha_{0}$ from $d$ pinches off $R_{1} R_{2} R_{3}$ from the annulus $R_{8} R_{9}$. In this extreme, $\theta_{1,2,3,8,9}^{d, a}(c)-\theta_{1,2,3,8,9}^{i, k}(c)$ approaches $\Theta_{8,9}^{d, b}-\Theta_{8,9}^{i, k}<0$. Since the extremes are both negative, the mod 2 count of zeros, and thus the contribution of $R_{1} R_{2} R_{3} R_{8} R_{9}$ to $\widehat{\mathrm{CFDD}}\left(\mathcal{H}_{\mathrm{SG}}\right)$, is zero. An analogous argument shows that $R_{1} R_{2} R_{3} R_{9} R_{10}$ does not contribute with $\left(\rho_{1}, \rho_{2}, \rho_{3}\right)$. 
$R_{1} R_{2} R_{3} R_{8} R_{9}$ may also contribute with the Reeb chord sequence $\left(\rho_{123}\right)$. This contribution can be checked with the $\mathcal{A}_{\infty}$ trick, using the relation for ( $\left.e d i, \rho_{1}, \rho_{23}\right)$. This relation implies that $m\left(e d i, \rho_{123}\right)=m\left(m\left(e d i, \rho_{1}\right), \rho_{23}\right)$. The only domain that could contribute a nontrivial operation $m\left(e d i, \rho_{1}\right)$ is $R_{3} R_{8} R_{9}$. As discussed above, $R_{3} R_{8} R_{9}$ does not contribute for our choice of complex structure $J$, and therefore $R_{1} R_{2} R_{3} R_{8} R_{9}$ does not contribute with $\vec{\rho}=\left(\rho_{123}\right)$. An analogous argument also shows that $R_{1} R_{2} R_{3} R_{9} R_{10}$ does not contribute with ( $\left.\rho_{123}\right)$.

The domain $R_{4} R_{5} R_{6} R_{8} R_{9}$ follows the same pattern. With Reeb chords $\left(\sigma_{1}, \sigma_{2}, \sigma_{3}\right)$ it is an annulus, and cuts in either direction split off the annulus $R_{8} R_{9}$ or the annulus $R_{4} R_{5} R_{8} R_{9}$. As we cut along $\beta_{0}$, we have that $\theta_{4,5,6,8,9}^{l, k}(c)-\theta_{4,5,6,8,9}^{d, b}$ approaches $\Theta_{8,9}^{i, k}-\Theta_{8,9}^{d, b}>0$. As we cut along $\alpha_{1}^{\sigma}$ toward the $\sigma$ boundary, $\theta_{4,5,6,8,9}^{l, k}(c)$ approaches $\infty$, and so $\theta_{4,5,6,8,9}^{l, k}(c)-\theta_{4,5,6,8,9}^{d, b}$ becomes positive. As a result, there is no contribution to the differential. The $\mathcal{A}_{\infty}$ relation for $\left(e d l, \sigma_{12}, \sigma_{3}\right)$ reveals that $R_{4} R_{5} R_{6} R_{8} R_{9}$ also does not contribute with $\left(\sigma_{123}\right)$. A similar argument shows that the domain $R_{4} R_{5} R_{6} R_{9} R_{10}$ does not contribute with either compatible sequence of Reeb chords.

Corners Consider the domain $R_{1} R_{2} R_{3} R_{4} R_{5} R_{8} R_{9}$, which connects $e d l$ to ahl . Any compatible sequence of Reeb chords must contain $\left(\sigma_{1}, \sigma_{2}\right)$, since $\left(\sigma_{12}\right)$ would not be strongly boundary monotonic with respect to the $\sigma$ boundary. For the domain to have the chords $\left(\sigma_{1}, \sigma_{2}\right)$ along the $\sigma$ boundary, there must be a cut along $\alpha_{1}^{\sigma}$. However, such a cut would leave corners at the point $c$. Since neither the initial generator $e d l$ nor the final generator $a h l$ contain $c$, it is impossible to have a corner at $c$. As a result, the domain $R_{1} R_{2} R_{3} R_{4} R_{5} R_{8} R_{9}$ can not contribute to $\widehat{\operatorname{CFDD}}\left(\mathcal{H}_{\mathrm{SG}}\right)$. The same reasoning applies to the domains $R_{2} R_{4} R_{5} R_{8} R_{9}$ and $R_{3} R_{4} R_{5} R_{8} R_{9}$.

Similarly, $R_{1} R_{2} R_{3} R_{5} R_{6} R_{9} R_{10}$ is only compatible with Reeb chord sequences containing $\left(\sigma_{2}, \sigma_{3}\right)$. This Reeb chord sequence requires a cut along $\alpha_{1}^{\sigma}$, which leaves corners at the point $g$. Since the initial generator $e b i$ and the final generator $a f i$ do not contain $g$, this domain can not contribute to the differential. The same is true for the domains $R_{1} R_{5} R_{6} R_{9} R_{10}$ and $R_{2} R_{5} R_{6} R_{9} R_{10}$, so these also do not contribute.

The domain $R_{2} R_{5} R_{8} R_{9} R_{9} R_{10} R_{11}$ connects the generators $a h i$ and $e n c$. However, there is no way to piece together these seven regions without having corners at points other than $a, h, i, e, n$ and $c$. Therefore, this domain can not contribute to the differential.

$\boldsymbol{R}_{\mathbf{1}} \boldsymbol{R}_{\mathbf{2}} \boldsymbol{R}_{\mathbf{3}} \boldsymbol{R}_{\mathbf{4}} \boldsymbol{R}_{\mathbf{5}} \boldsymbol{R}_{\mathbf{6}} \boldsymbol{R}_{\mathbf{8}} \boldsymbol{R}_{\mathbf{9}} \boldsymbol{R}_{10}$ This domain has four compatible sequences of Reeb chords. It is possible to use $\mathcal{A}_{\infty}$ relations and analyze the contribution of each one. However, it is easier to notice that this domain contributes if an only if the shaded 


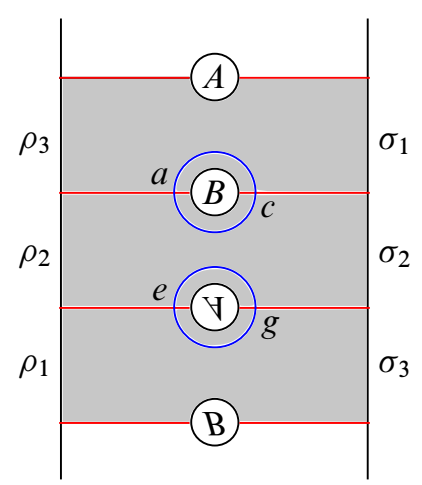

Figure 18: A bordered Heegaard diagram for the identity bimodule, $\widehat{\mathrm{CFDD}}(\mathbb{I})$. $R_{1} R_{2} R_{3} R_{4} R_{5} R_{6} R_{8} R_{9} R_{10}$ contributes to $\widehat{\mathrm{CFDD}}\left(\mathcal{H}_{\mathrm{SG}}\right)$ if and only if the shaded region contributes to the differential from ec to $a g$ in $\widehat{\mathrm{CFDD}}(\mathbb{I})$.

domain contributes in the Heegaard diagram for the mapping cylinder of the identity map shown in Figure 18. The computation of $\widehat{\operatorname{CFDD}}(\mathbb{I})$ in [10, Proposition 10.1] reveals that this domain must contribute.

Using $\partial^{\mathbf{2}}=\mathbf{0}$ We can deduce the contribution of other domains using the fact that $\widehat{\mathrm{CFDD}}\left(\mathcal{H}_{\mathrm{SG}}\right)$ must satisfy $\partial^{2}=0$. We now have enough information to deduce the contribution of all domains but one using $\partial^{2}$. Table 5 gives the differential on $\widehat{\mathrm{CFDD}}\left(\mathcal{H}_{\mathrm{SG}}\right)$ as computed so far, with coefficients $\lambda_{i_{1}, \ldots, i_{k}}$ representing unknown contributions; $\lambda_{i_{1}, \ldots, i_{k}}$ is 1 if the domain $R_{i_{1}} \cdots R_{i_{k}}$ contributes to the differential and 0 otherwise.

Consider the generator $a h j$. We have $\partial(a h j)=\sigma_{1}(a h i)+\lambda_{4,8,9,10,11} \sigma_{1}(a n g)$, and so

$$
\begin{aligned}
0= & \partial^{2}(a h j) \\
= & \sigma_{1}\left(\lambda_{2,3,5,8,9,9,10} \rho_{23} \sigma_{2}(a f l)+\lambda_{2,5,6,8,9,9,10} \rho_{2} \sigma_{23}(e b k)\right) \\
& +\lambda_{4,8,9,10,11} \sigma_{1}\left(\rho_{2} \sigma_{2}(e n c)\right) .
\end{aligned}
$$

It follows that $\lambda_{2,3,5,8,9,9,10}=\lambda_{2,5,6,8,9,9,10}=\lambda_{4,8,9,10,11}=0$. Thus we find that

$$
\begin{aligned}
& 0= \partial^{2}(e d i) \\
&= \rho_{1}(0)+\lambda_{1,8,9,10,11} \rho_{1}\left(\rho_{2} \sigma_{2}(e n c)\right) \\
& \quad+\lambda_{1,2,5,8,9,9,10} \rho_{12} \sigma_{2}\left(\rho_{3}(\text { afl })+\sigma_{123}(e b i)+\sigma_{3}(e b k)+(e n c)\right) \\
&=\left(\lambda_{1,8,9,10,11}+\lambda_{1,2,5,8,9,9,10} \rho_{12} \sigma_{2}(e n c)\right. \\
& \quad
\end{aligned}
$$

The coefficient of afl implies that $\lambda_{1,2,5,8,9,9,10}=0$, and the coefficient of enc implies that $\lambda_{1,8,9,10,11}=0$. 


$$
\begin{aligned}
& \partial(a f i)=\rho_{2}(e d i) \\
& \partial(a f j)=\sigma_{1}(a f i)+\rho_{2}(e d j) \\
& \partial(a f k)=\sigma_{2}(a f j)+\rho_{2}(e d k) \\
& \partial(a f l)=\sigma_{123}(a f i)+\sigma_{3}(a f k)+\sigma_{1} a n g+\rho_{2} e d l
\end{aligned}
$$

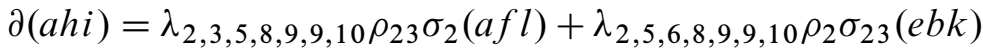

$$
\begin{aligned}
& \partial(a h j)=\sigma_{1}(a h i)+\lambda_{4,8,9,10,11} \sigma_{1}(a n g) \\
& \partial(a h k)=\sigma_{2}(a h j) \\
& \partial(a h l)=\sigma_{123}(a h i)+\sigma_{3}(a h k)+\lambda_{2,3,4,5,6,8,9} \rho_{23} \sigma_{123}(a h k) \\
& +\lambda_{4,5,6,8,9,10,11} \sigma_{123} \text { (ang) } \\
& \partial(\text { ang })=\rho_{2} \sigma_{2}(\text { enc }) \\
& \partial(a m g)=\sigma_{23}(a f i)+(a h i)+\rho_{23}(a h k)+\lambda_{7,8,9,10,11}(a n g)+(a n g) \\
& +\lambda_{2,5,7,8,9,9,10} \rho_{2} \sigma_{2}(e b l)+\rho_{2} \sigma_{2}(e m c) \\
& \partial(e b i)=\rho_{3}(a f i)+\lambda_{1,2,3,8,9,10,11} \rho_{123}(\text { ang }) \\
& \partial(e b j)=\rho_{3}(a f j)+\sigma_{1}(e b i)+\lambda_{1,2,4,5,6,9,10} \rho_{12} \sigma_{123}(e b i)+\rho_{12}(e n c) \\
& \partial(e b k)=\rho_{3}(a f k)+\rho_{1}(a n g)+\sigma_{2}(e b j) \\
& \partial(e b l)=\rho_{3}(a f l)+\sigma_{123}(e b i)+\sigma_{3}(e b k)+(e n c) \\
& \partial(e d i)=\rho_{1}(a h i)+\lambda_{1,8,9,10,11} \rho_{1}(\text { ang })+\lambda_{1,2,5,8,9,9,10} \rho_{12} \sigma_{2}(e b l) \\
& \partial(e d j)=\rho_{1}(a h j)+\sigma_{1}(e d i) \\
& \partial(e d k)=\rho_{1}(a h k)+\sigma_{2}(e d j) \\
& \partial(e d l)=\rho_{1}(a h l)+\sigma_{123}(e d i)+\sigma_{3}(e d k)+\sigma_{12}(e n c) \\
& \partial(\text { enc })=\rho_{3} \sigma_{1}(\text { ang })+\rho_{1} \sigma_{3}(\text { ang })+\rho_{123} \sigma_{123}(\text { ang }) \\
& \partial(e m c)=\lambda_{3,7,8,9,10} \rho_{3}(a f l)+\rho_{3}(a h j)+\lambda_{1,2,3,7,8,9,10} \rho_{123}(a h l)+\rho_{3} \sigma_{1}(a m g) \\
& +\rho_{1} \sigma_{3}(\mathrm{amg})+\rho_{123} \sigma_{123}(\mathrm{amg})+\lambda_{4,5,6,7,8,9,10} \sigma_{123}(\mathrm{ebi}) \\
& +\lambda_{6,7,8,9,10} \sigma_{3}(e b k)+\sigma_{3}(e d i)+\lambda_{7,8,9,10,11}(e n c)+(e n c)
\end{aligned}
$$

Table 5: The differential on $\widehat{\mathrm{CFDD}}\left(\mathcal{H}_{\mathrm{SG}}\right)$. Here $\lambda$ is used for coefficients that have yet to be determined; they are 0 or 1 depending on the contribution of the corresponding domain.

The coefficient of the enc term of $\partial^{2}(a m g)$ is $\lambda_{2,5,7,8,9,9,10} \rho_{2} \sigma_{2}$, which implies that $\lambda_{2,5,7,8,9,9,10}=0$. Then the afl term of $\partial^{2}(a m g)$ becomes $\lambda_{3,7,8,9,10} \rho_{23} \sigma_{2}(a f l)$, and the ebk term becomes $\lambda_{6,7,8,9,10} \rho_{2} \sigma_{23}(e b k)$; thus $\lambda_{3,7,8,9,10}=\lambda_{6,7,8,9,10}=0$. Similarly the ang term of $\partial^{2}(e d l)$ reveals that $\lambda_{4,5,6,8,9,10,11}=1$ and the ahk 


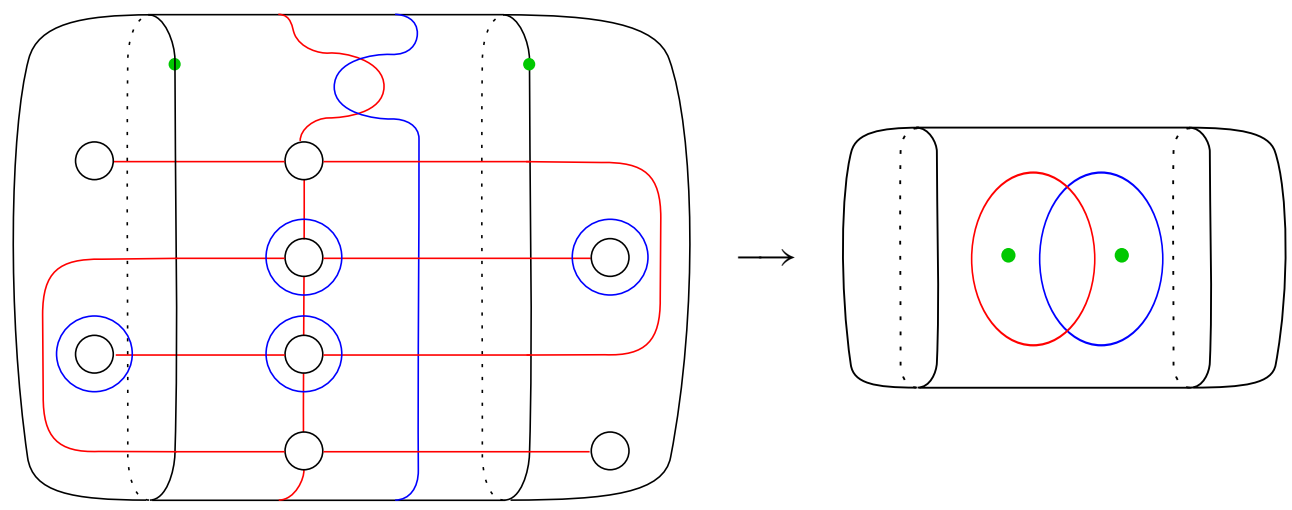

Figure 19: The manifold obtained from the self-gluer by capping off both ends with identical 0 -framed solid tori

term of $\partial^{2}(e d l)$ implies that $\lambda_{2,3,4,5,6,8,9}=0$. The af $i$ term of $\partial^{2}(e b j)$ implies that $\lambda_{1,2,4,5,6,9,10}=0$, and the ang term implies that $\lambda_{1,2,3,8,9,10,11}=1$. Finally, the ang term of $\partial^{2}$ (emc) implies that $\lambda_{4,5,6,7,8,9,10}=1$, and the ahi term implies that $\lambda_{1,2,3,7,8,9,10}=1$. The only coefficient in Table 5 that remains undetermined is $\lambda_{7,8,9,10,11}$.

$\boldsymbol{R}_{\mathbf{7}} \boldsymbol{R}_{\mathbf{8}} \boldsymbol{R}_{\mathbf{9}} \boldsymbol{R}_{10} \boldsymbol{R}_{11}$ We have determined that $\widehat{\mathrm{CFDD}}\left(\mathcal{H}_{\mathrm{SG}}\right)$ is one of two possibilities, depending on the value of $\lambda_{7,8,9,10,11}$. We will deduce the right choice by showing that one of these possible bimodules does not behave correctly under tensoring with type $A$ modules for the solid torus.

Consider the closed, doubly basepointed Heegaard diagram in Figure 19 (left), which is obtained from the bordered Heegaard diagram $\mathcal{H}_{\mathrm{SG}}$ by gluing bordered Heegaard diagrams for solid tori to each boundary component. A sequence of isotopies and destabilizations leads to the diagram in Figure 19 (right), so it is easy to check that $\widehat{\mathrm{HF}}$ of the manifold represented by this diagram has rank 2 . Also, $\widehat{\mathrm{HF}}$ can be obtained by taking the box tensor product of $\widehat{\mathrm{CFDD}}\left(\mathcal{H}_{\mathrm{SG}}\right)$ with two copies of the type $A$ module for the solid torus. A bounded version of the solid torus module has three generators $x, y$ and $z$ and the following operations:

$$
m_{1}(x)=z, \quad m_{2}\left(x, \rho_{1}\right)=y, \quad m_{2}\left(x, \rho_{2}\right)=z, \quad m_{2}\left(x, \rho_{12}\right)=z .
$$

It is routine to perform the tensor products, and we find that the homology of the resulting chain complex is rank 2 if $\lambda_{7,8,9,10,11}=1$, and rank 4 if $\lambda_{7,8,9,10,11}=0$. Thus, the domain $R_{7} R_{8} R_{9} R_{10} R_{11}$ must contribute, and we have completed the 


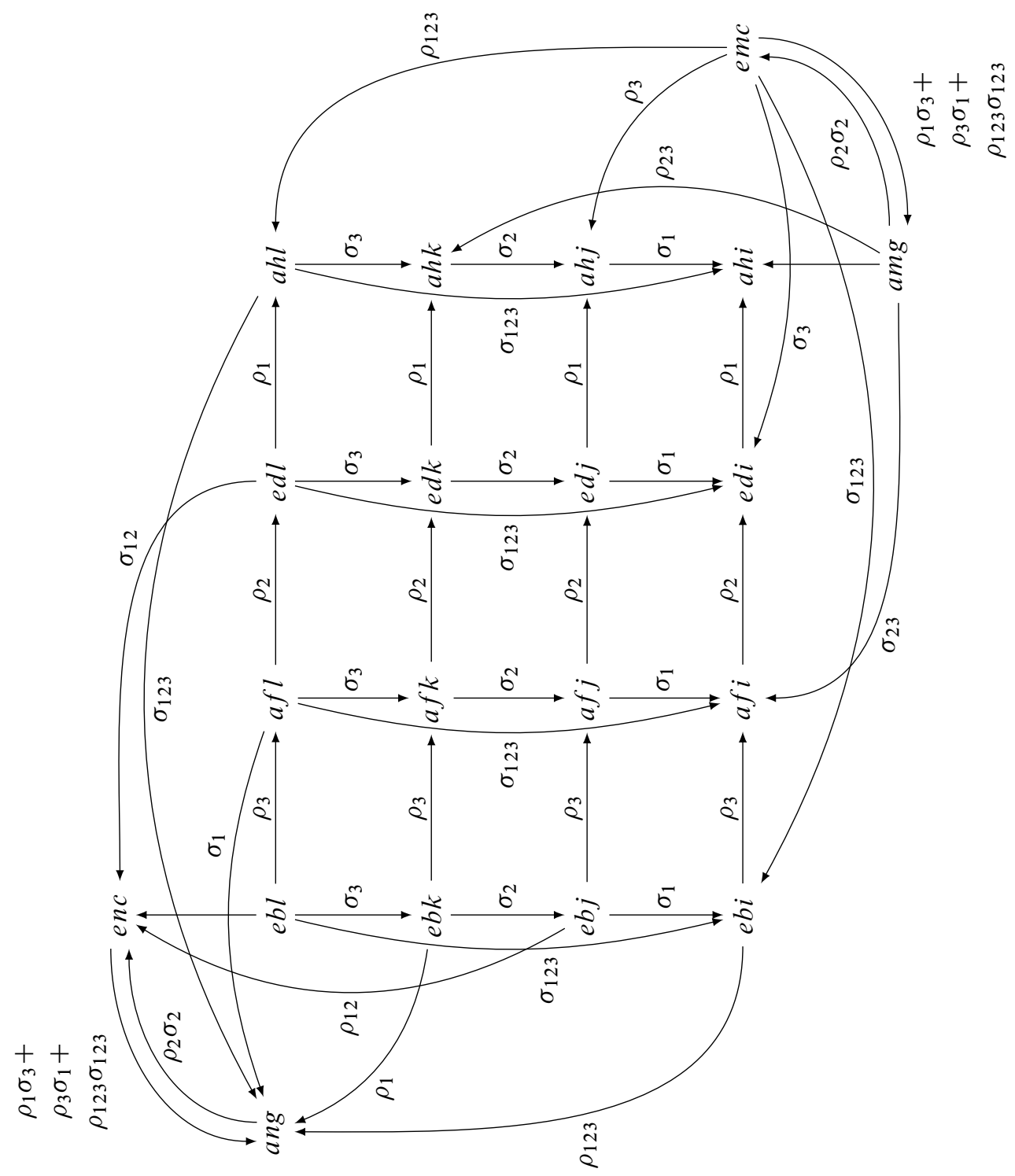

Figure 20: $\widehat{\mathrm{CFDD}}\left(\mathcal{H}_{\mathrm{SG}}\right)$ in the middle $\operatorname{spin}^{c}$-structure

computation of $\widehat{\mathrm{CFDD}}\left(\mathcal{H}_{\mathrm{SG}}\right)$ in the middle $\operatorname{spin}^{c}$-structure. The result is pictured in Figure 20.

Extremal spin ${ }^{c}$-structures First consider the $\operatorname{spin}^{c}$-structure in which both $\alpha^{\rho}$ arcs are occupied and neither $\alpha^{\sigma}$ arc is occupied. There are only two generators with those 
conditions: aem and aen. There are two domains which have the right corner count to connect aem and aen. The bigon $R_{12}$ contributes a differential from aem to aen. The domain $R_{7} R_{8} R_{9} R_{10} R_{11}$, as an element of $\pi_{2}$ (aem,aen), has index -1 and thus does not contribute. Canceling the differential and two generators, we find that $\widehat{\mathrm{CFDD}}\left(\mathcal{H}_{\mathrm{SG}}\right)$ in this extremal $\operatorname{spin}^{c}$-structure has no generators.

The other extremal $\operatorname{spin}^{c}$-structure has more generators (given the choice of Heegaard diagram $\mathcal{H}_{\mathrm{SG}}$ ), but the corresponding summand of $\widehat{\mathrm{CFDD}}\left(\mathcal{H}_{\mathrm{SG}}\right)$ is still trivial. Indeed, we could handleslide $\beta_{0}$ across the handles in Figure 15 to produce a new Heegaard diagram with only two generators in this $\operatorname{spin}^{c}$-structure. This diagram is a mirror image of $\mathcal{H}_{\mathrm{SG}}$, so the reasoning above applies and shows that the two generators are canceled by the single differential between them.

\section{A Gradings}

As described in Section $2 \mathrm{H}, \widehat{\mathrm{CFDD}}\left(\mathcal{H}_{\mathrm{SG}}\right)$ is graded by a set which is a quotient of the noncommutative group $G_{2,0}$. We will compute this (relative) grading using Figure 20.

We choose $e b l$ to be the preferred generator and set $\operatorname{gr}(e b l)=\overrightarrow{0}=(0 ; 0,0 ; 0,0)$. The arrow labeled $\rho_{3}$ from $e b l$ to $a f l$ determines the grading of $a f l$ :

$$
\operatorname{gr}(a f l)=\lambda^{-1} \operatorname{gr}\left(\rho_{3}\right)^{-1} \operatorname{gr}(e b l)=\lambda^{-1}\left(\frac{1}{2} ; \frac{1}{2},-\frac{1}{2} ; 0,0\right) \overrightarrow{0}=\left(-\frac{1}{2} ; \frac{1}{2},-\frac{1}{2} ; 0,0\right) .
$$

Similarly, the successive arrows labeled $\rho_{2}$ and $\rho_{1}$ (moving right from $a f l$ in Figure 20) determine the gradings of $e d l$ and $a h l$ :

$$
\begin{aligned}
\operatorname{gr}(e d l) & =\lambda^{-1} \operatorname{gr}\left(\rho_{2}\right)^{-1} \operatorname{gr}(\text { afl })=\lambda^{-1}\left(\frac{1}{2} ;-\frac{1}{2},-\frac{1}{2} ; 0,0\right)\left(-\frac{1}{2} ; \frac{1}{2},-\frac{1}{2} ; 0,0\right) \\
& =\left(-\frac{1}{2} ; 0,-1 ; 0,0\right), \\
\operatorname{gr}(a h l) & =\lambda^{-1} \operatorname{gr}\left(\rho_{1}\right)^{-1} \operatorname{gr}(e d l)=\lambda^{-1}\left(\frac{1}{2} ;-\frac{1}{2}, \frac{1}{2} ; 0,0\right)\left(-\frac{3}{2} ; 0,-1 ; 0,0\right) \\
& =\left(-\frac{1}{2} ;-\frac{1}{2},-\frac{1}{2} ; 0,0\right) .
\end{aligned}
$$

Working down the chain of $\sigma$ labeled arrows starting from $e b l$ determines the gradings of $e b k, e b j$ and $e b i$ :

$$
\begin{aligned}
& \operatorname{gr}(e b k)=\lambda^{-1} \operatorname{gr}\left(\sigma_{3}\right)^{-1} \operatorname{gr}(e b l)=\left(-\frac{1}{2} ; 0,0 ; \frac{1}{2},-\frac{1}{2}\right), \\
& \operatorname{gr}(e b j)=\lambda^{-1} \operatorname{gr}\left(\sigma_{2}\right)^{-1} \operatorname{gr}(e b k)=\left(-\frac{1}{2} ; 0,0 ; 0,-1\right), \\
& \operatorname{gr}(e b i)=\lambda^{-1} \operatorname{gr}\left(\sigma_{1}\right)^{-1} \operatorname{gr}(e b j)=\left(-\frac{1}{2} ; 0,0 ;-\frac{1}{2},-\frac{1}{2}\right) .
\end{aligned}
$$


The vertical chains of $\sigma$ labeled arrows from $a f l$, edl and $a h l$ determine the gradings

$$
\begin{aligned}
& \operatorname{gr}(\text { afk })=\lambda^{-1} \operatorname{gr}\left(\sigma_{3}\right)^{-1} \operatorname{gr}(\text { afl })=\left(-1 ; \frac{1}{2},-\frac{1}{2} ; \frac{1}{2},-\frac{1}{2}\right), \\
& \operatorname{gr}(a f j)=\lambda^{-1} \operatorname{gr}\left(\sigma_{2}\right)^{-1} \operatorname{gr}(a f k)=\left(-1 ; \frac{1}{2},-\frac{1}{2} ; 0,-1\right), \\
& \operatorname{gr}(\text { afi })=\lambda^{-1} \operatorname{gr}\left(\sigma_{1}\right)^{-1} \operatorname{gr}(a f j)=\left(-1 ; \frac{1}{2},-\frac{1}{2} ;-\frac{1}{2},-\frac{1}{2}\right), \\
& \operatorname{gr}(e d k)=\lambda^{-1} \operatorname{gr}\left(\sigma_{3}\right)^{-1} \operatorname{gr}(e d l)=\left(-1 ; 0,-1 ; \frac{1}{2},-\frac{1}{2}\right), \\
& \operatorname{gr}(e d j)=\lambda^{-1} \operatorname{gr}\left(\sigma_{2}\right)^{-1} \operatorname{gr}(e d k)=(-1 ; 0,-1 ; 0,-1), \\
& \operatorname{gr}(e d i)=\lambda^{-1} \operatorname{gr}\left(\sigma_{1}\right)^{-1} \operatorname{gr}(e d j)=\left(-1 ; 0,-1 ;-\frac{1}{2},-\frac{1}{2}\right), \\
& \operatorname{gr}(a h k)=\lambda^{-1} \operatorname{gr}\left(\sigma_{3}\right)^{-1} \operatorname{gr}(a h l)=\left(-1 ;-\frac{1}{2},-\frac{1}{2} ; \frac{1}{2},-\frac{1}{2}\right), \\
& \operatorname{gr}(a h j)=\lambda^{-1} \operatorname{gr}\left(\sigma_{2}\right)^{-1} \operatorname{gr}(a h k)=\left(-1 ;-\frac{1}{2},-\frac{1}{2} ; 0,-1\right), \\
& \operatorname{gr}(a h i)=\lambda^{-1} \operatorname{gr}\left(\sigma_{1}\right)^{-1} \operatorname{gr}(a h j)=\left(-1 ;-\frac{1}{2},-\frac{1}{2} ;-\frac{1}{2},-\frac{1}{2}\right) .
\end{aligned}
$$

The two unlabeled arrows in the diagram determine the gradings of enc and amg:

$$
\begin{aligned}
\operatorname{gr}(e n c) & =\lambda^{-1} \operatorname{gr}(e b l)=(-1 ; 0,0 ; 0,0), \\
\operatorname{gr}(a m g) & =\lambda \operatorname{gr}(a h i)=\left(0 ;-\frac{1}{2},-\frac{1}{2} ;-\frac{1}{2},-\frac{1}{2}\right) .
\end{aligned}
$$

Finally, the two arrows labeled $\rho_{2} \sigma_{2}$ determine the gradings of ang and emc:

$$
\begin{aligned}
& \operatorname{gr}(\text { ang })=\lambda \operatorname{gr}\left(\rho_{2}\right) \operatorname{gr}\left(\sigma_{2}\right) \operatorname{gr}(\text { enc })=\left(-1 ; \frac{1}{2}, \frac{1}{2} ; \frac{1}{2}, \frac{1}{2}\right), \\
& \operatorname{gr}(\text { emc })=\lambda \operatorname{gr}\left(\rho_{2}\right) \operatorname{gr}\left(\sigma_{2}\right) \operatorname{gr}(a m g)=(0 ; 0,0 ; 0,0) .
\end{aligned}
$$

It remains to compute the indeterminacy $\mathcal{P}(e b l)$. We compute equivalent values for the grading of $e b l$ by using the loop ebl to $a f l$ to $e d l$ to $e n c$ to $e b l$ and the loop $e b l$ to $e b k$ to $e b j$ to $e n c$ to $e b l$. The first loop gives the element of $\mathcal{P}(e b l)$ corresponding to a periodic domain with boundary $\rho_{23}+\sigma_{12}$ :

$$
\begin{aligned}
& \operatorname{gr}(a f l)=\lambda^{-1} \operatorname{gr}\left(\rho_{3}\right)^{-1} \operatorname{gr}(e b l)=\left(-\frac{1}{2} ; \frac{1}{2},-\frac{1}{2} ; 0,0\right), \\
& \operatorname{gr}(e d l)=\lambda^{-1} \operatorname{gr}\left(\rho_{2}\right)^{-1} \operatorname{gr}(a f l)=\left(-\frac{1}{2} ; 0,-1 ; 0,0\right), \\
& \operatorname{gr}(e n c)=\lambda^{-1} \operatorname{gr}\left(\sigma_{12}\right)^{-1} \operatorname{gr}(a f l)=(-1 ; 0,-1 ;-1,0), \\
& \operatorname{gr}(e d l)=\lambda \operatorname{gr}(e n c)=(0 ; 0,-1 ;-1,0) .
\end{aligned}
$$

The second loop gives the element of $\mathcal{P}(e b l)$ corresponding to a periodic domain with boundary $\rho_{12}+\sigma_{23}$ :

$$
\begin{aligned}
& \operatorname{gr}(e b k)=\lambda^{-1} \operatorname{gr}\left(\sigma_{3}\right)^{-1} \operatorname{gr}(e b l)=\left(-\frac{1}{2} ; 0,0 ; \frac{1}{2},-\frac{1}{2}\right), \\
& \operatorname{gr}(e b j)=\lambda^{-1} \operatorname{gr}\left(\sigma_{2}\right)^{-1} \operatorname{gr}(e b k)=\left(-\frac{1}{2} ; 0,0 ; 0,-1\right), \\
& \operatorname{gr}(e n c)=\lambda^{-1} \operatorname{gr}\left(\rho_{12}\right)^{-1} \operatorname{gr}(e b j)=(-1 ;-1,0 ; 0,-1), \\
& \operatorname{gr}(e d l)=\lambda \operatorname{gr}(e n c)=(0 ;-1,0 ; 0,-1) .
\end{aligned}
$$

Thus $\mathcal{P}(e b l)$ is the subgroup of $G_{2,0}$ generated by $(0 ; 0,-1 ;-1,0)$ and $(0 ;-1,0 ; 0,-1)$. 


\section{Computing $\widehat{\mathrm{HF}}$ of graph manifolds}

This section describes the procedure for computing $\widehat{\mathrm{HF}}$ of an arbitrary graph manifold given a connected plumbing graph $\Gamma$. For simplicity, we will assume that every vertex of $\Gamma$ has nonnegative genus. The manifold can be constructed from simpler bordered pieces using two types of gluing: extension glues fibers to fibers and base surface to base surface, and plumbing glues a fiber of one bundle to a curve in the base of the other bundle. Gluing two $S^{1}$-bundles by extension produces an $S^{1}$-bundle over the surface obtained by gluing the two bases.

In the Heegaard diagram for $\mathcal{Y}_{\mathcal{P}}$, recall that $\alpha_{1}^{\rho}, \alpha_{2}^{\sigma}$ and $\alpha_{1}^{\tau}$ parametrize curves in the base surface $\mathcal{P}$, while $\alpha_{2}^{\rho}, \alpha_{1}^{\sigma}$ and $\alpha_{2}^{\tau}$ parametrize fibers. If we glue two type $D$ boundaries together, $\alpha_{1}$ glues to $\alpha_{2}$ and vice versa (to combine the relevant modules we would first change one of the boundaries to type $A$, which switches $\alpha_{1}$ and $\alpha_{2}$ ). Thus gluing the $\rho$ boundary of one copy of $\mathcal{Y}_{\mathcal{P}}$ to the $\sigma$ boundary of another is extension. Gluing the $\rho$ boundary to the $\tau$ boundary is plumbing.

It will be convenient to introduce the bordered manifold $\overline{\mathcal{Y}}_{\mathcal{P}}$, the mirror image of $\mathcal{Y}_{\mathcal{P}}$. The trimodule $\widehat{\mathrm{CFD}}^{3}\left(\overline{\mathcal{Y}}_{\mathcal{P}}\right)$ can be obtained from $\widehat{\mathrm{CFD}}^{3}\left(\mathcal{Y}_{\mathcal{P}}\right)$ by interchanging ones with threes for all algebra elements and reversing the direction of the arrows. Also, $\alpha_{1}$ and $\alpha_{2}$ are interchanged on each boundary component.

\section{A Trivial bundles over surfaces}

Recall that each vertex of $\Gamma$ represents a particular $S^{1}$-bundle over a surface $S_{g, b}$ with genus $g$ and $b$ boundary components. We first construct the trivial bundle over $S_{g, b}$. If $g=0$ and $b \geq 3$, then we simply glue copies of $\mathcal{Y}_{\mathcal{P}}$ by extension until we have the right number of boundary components. The multimodule $\widehat{\mathrm{CFD}}^{b}$ is obtained by taking box tensor products, inserting copies of $\widehat{\mathrm{CFAA}}(\mathbb{I})$ when two type $D$ boundaries are glued. For instance, $\widehat{\mathrm{CFD}}^{4}\left(S^{1} \times S_{0,4}\right)$ is given by

$$
\left(\widehat{\mathrm{CFAA}}(\mathbb{I}) \otimes \widehat{\mathrm{CFD}}^{3}\left(\mathcal{Y}_{\mathcal{P}}\right)\right) \otimes \widehat{\mathrm{CFD}}^{3}\left(\mathcal{Y}_{\mathcal{P}}\right),
$$

where the tensor products are with respect to the $\rho$ and $\sigma$ boundaries on the two copies of $\widehat{\mathrm{CFD}}^{3}\left(\mathcal{Y}_{\mathcal{P}}\right)$. The trivial bundle over $S_{0,1}$ is just the solid torus, which has bordered invariant

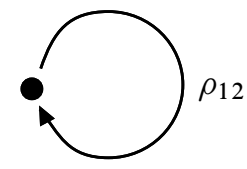

or

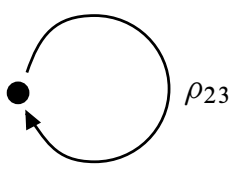




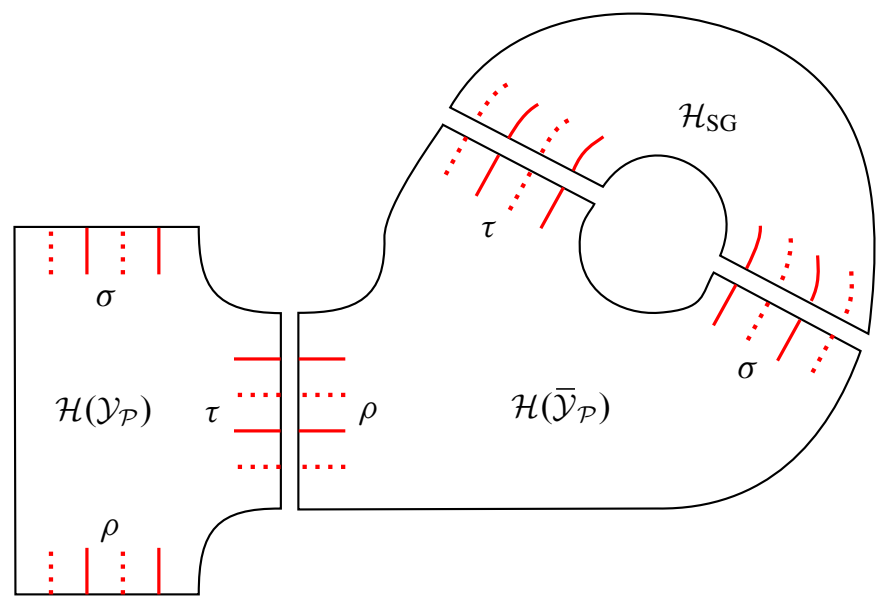

Figure 21: A Heegaard diagram for $S_{1,2} \times S^{1}$ can be obtained by gluing $\mathcal{H}_{\mathrm{SG}}$ and Heegaard diagrams for $\mathcal{Y}_{\mathcal{P}}$ and $\overline{\mathcal{Y}}_{\mathcal{P}}$. Solid red lines indicate $\alpha$ arcs in the base of each bundle, while dotted red lines correspond to fibers.

depending on whether $\alpha_{1}$ parametrizes a curve in the base (left) or a fiber (right) [8, Section 11.2]. The trivial bundle over $S_{0,2}$ is the same as the mapping cylinder of the identity map on the torus. The corresponding bimodule $\widehat{\operatorname{CFDD}}(\mathbb{I})$ is computed in $[10$, Proposition 10.1]. Here either $\alpha$ arc can be the fiber, but $\alpha_{1}$ on one boundary is the same as $\alpha_{2}$ on the other boundary.

We construct a Heegaard diagram for the trivial bundle over $S_{1,2}$ as indicated in Figure 21. Notice that we must insert a copy of $\mathcal{H}_{\mathrm{SG}}$ when we glue two components of $\partial \overline{\mathcal{Y}}_{\mathcal{P}}$ to each other. The bimodule $\widehat{\operatorname{CFDD}}\left(S^{1} \times S_{1,2}\right)$ can be computed as follows:

- Change $\widehat{\mathrm{CFD}}^{3}\left(\overline{\mathcal{Y}}_{\mathcal{P}}\right)$ to a type $D D A$ trimodule by tensoring with $\widehat{\mathrm{CFAA}}(\mathbb{I})$ along the $\sigma$ boundary.

- Tensor the type $A$ boundary of the resulting trimodule with $\widehat{\mathrm{CFDD}}\left(\mathcal{H}_{\mathrm{SG}}\right)$.

- Change the $\tau$ boundary to type $A$ by tensoring with $\widehat{\mathrm{CFAA}}(\mathbb{I})$, and then take the Hochschild homology with respect to the appropriate boundary components, resulting in a type $D$ module.

- Change this module to type $A$ by tensoring with $\widehat{\mathrm{CFAA}}(\mathbb{I})$ and tensor with the $\tau$ boundary of $\widehat{\operatorname{CFD}}^{3}\left(\mathcal{Y}_{\mathcal{P}}\right)$.

- The result is a type $D D$ bimodule with 16 generators. Note that it is still the case that $\alpha_{2}^{\rho}$ and $\alpha_{1}^{\sigma}$ represent fibers. 
For $b>0$, the trivial bundle over $S_{g, b}$ can now be obtained easily by extending $S_{0, b}$ with $g$ copies of $S_{1,2}$. For the case of $b=0$, we simply extend the trivial bundle over $S_{g, 1}$ by capping off the boundary with the trivial bundle over $S_{0,1}$.

\section{B Nontrivial bundles}

In general the bundle associated to a vertex of $\Gamma$ is nontrivial, with a specified Euler number $e$. The Euler number of a circle bundle over a surface with boundary is well defined only once a trivialization is chosen on the boundary circles. A trivialization of an $S^{1}$-bundle over a circle is simply a map $\phi$ from the standard torus $S^{1} \times S^{1}$ to the bundle. Choosing a trivialization amounts to specifying two curves, $\gamma_{f}=\phi\left(\{*\} \times S^{1}\right)$ and $\gamma_{b}=\phi\left(S^{1} \times\{*\}\right)$. We say that $\gamma_{f}$ is a fiber and $\gamma_{b}$ is a curve in the base surface.

The Euler number for $S^{1}$-bundles over surfaces with boundary is additive under gluing (when the gluing identifies fibers with fibers and base curves with base curves). Thus to understand bundles with arbitrary Euler number it is sufficient to find Euler number \pm 1 bundles over the annulus. Gluing these to a given $S^{1}$-bundle over a surface adds \pm 1 to the Euler number without changing the topology of the base surface.

Given a torus $T^{2}=S^{1} \times S^{1}$, let $a$ denote the curve $\{*\} \times S^{1}$ and let $b$ denote the curve $S^{1} \times\{*\}$. Let $\tau_{a}^{-1}: T^{2} \rightarrow T^{2}$ denote the negative Dehn twist about $a$ and consider the mapping cylinder $M_{\tau_{a}^{-1}}$. Now $M_{\tau_{a}^{-1}}$ is a $T^{2}$ bundle over $[0,1]$, but there is also a projection of each fiber to $S^{1}$ (namely, projection to the first $S^{1}$ factor). As a result, we can also view $M_{\tau_{a}^{-1}}$ as an $S^{1}$ bundle over [0,1] $\times S^{1}$. We fix a trivialization of this bundle by letting $\left(\gamma_{f}, \gamma_{b}\right)$ be $(a, b)$ on the $\{0\} \times S^{1}$ end and $\left(\tau_{a}^{-1}(a), \tau_{a}^{-1}(b)\right)$ on the $\{1\} \times S^{1}$ end.

To compute the Euler number of this bundle, we will think of it as the boundary of $D^{2}$-bundle over $[0,1] \times S^{1}$. The Euler number is an obstruction to the existence of a nonzero section of this bundle; more precisely, it is the signed intersection number of the zero section of this $D^{2}$-bundle and any section transverse to the zero section. Figure 22 depicts a section of a $D^{2}$-bundle over $[0,1] \times S^{1}$. Over each point in the cylinder (the rectangle, with the top and bottom edges identified) we choose a point in the corresponding $D^{2}$ fiber (represented in the figure by a vector). The signed number of zeros of this section is one. It remains to check that the boundary of this $D^{2}$-bundle is $M_{\tau_{a}^{-1}}$.

Over each $x \in[0,1]$, restricting the $S^{1}$-bundle to $\{x\} \times S^{1}$ (that is, to a vertical line in Figure 22) gives a torus; we will identify this with $T^{2}$ so that $\{x\} \times S^{1}$ identifies with $b$ and a fiber over a point identifies with $a$. The section of the bundle specifies a trivialization over the boundary by taking $\gamma_{f}$ to be a fiber and $\gamma_{b}$ to be the image of the section. We see that on the left boundary $\gamma_{f}=a$ and $\gamma_{b}=b$, and on the right 

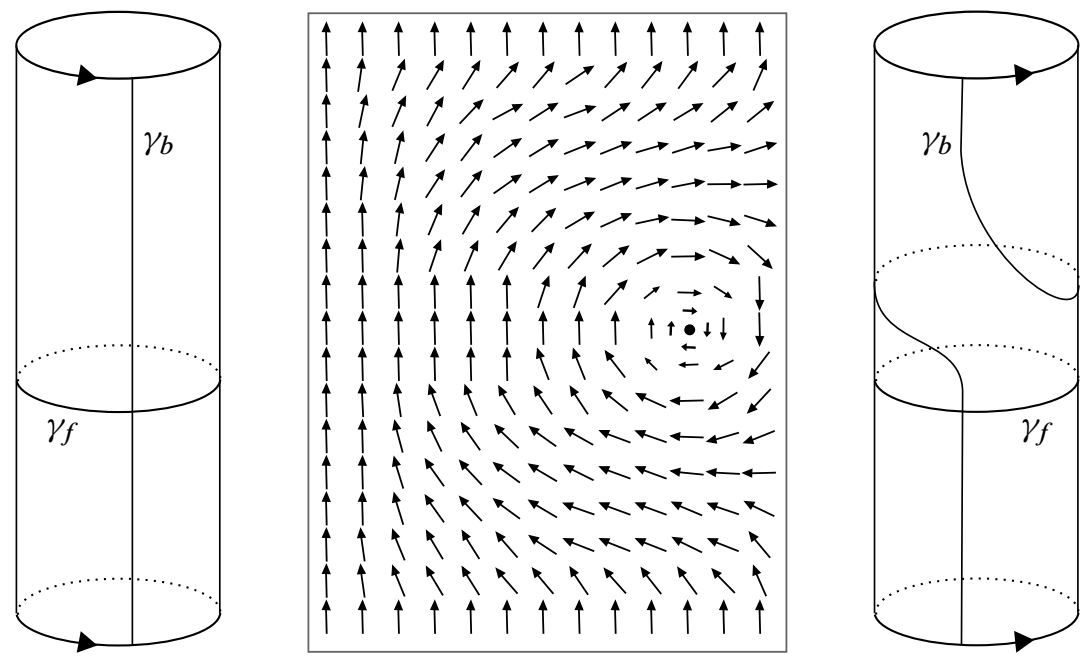

Figure 22: A section of a $D^{2}$-bundle over the cylinder (center), where the top and bottom edges are identified. The signed number of zeros indicates that the Euler number is +1 . Over each end of the cylinder, the section is nonzero and gives a section of the boundary $S^{1}$-bundle. This specifies a trivialization of the $S^{1}$-bundle over each end of the cylinder, which is pictured on the tori on the left and right.

boundary $\gamma_{f}=\tau_{a}^{-1}(a)=a$ and $\gamma_{b}=\tau_{a}^{-1}(b)$, so this bundle corresponds with the mapping cylinder $M_{\tau_{a}^{-1}}$.

Thus, to increase the Euler number of a given $S^{1}$-bundle over a surface, we attach a mapping cylinder of a negative Dehn twist about a curve which is identified with the fiber. In the same way, applying a positive Dehn twist about the fiber has the effect of decreasing the Euler number by 1 . The Euler number zero bundles constructed above always have fiber $\alpha_{1}$ or $\alpha_{2}$, and the bimodules for Dehn twists about $\alpha_{1}$ and $\alpha_{2}$ are known [10, Section 10.2]. By tensoring with enough of these bimodules we can obtain the bordered invariants for arbitrary $S^{1}$-bundles over arbitrary (oriented) surfaces with boundary.

\section{C Combining vertices}

Once multimodules have been determined for each vertex of $\Gamma$, they can be combined according to the edges of $\Gamma$. If vertices $v_{1}$ and $v_{2}$ are connected by an edge, choose a boundary component of each circle bundle such that both boundaries have fiber $\alpha_{1}$ or both have fiber $\alpha_{2}$. Take the box tensor product (after changing one boundary component to type $A$ ) to compute the new multimodule. If there is no way to choose a 

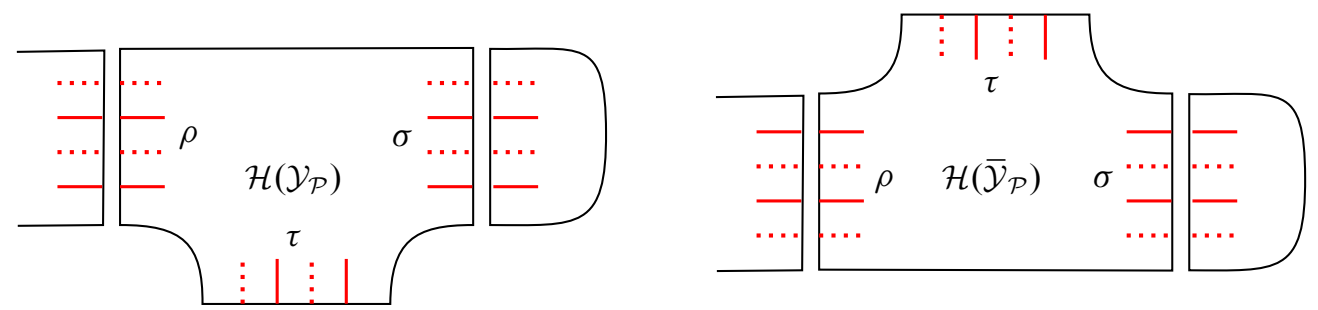

Figure 23: The fiber direction can be changed by extending at a boundary with $\mathcal{Y}_{\mathcal{P}}$ or $\overline{\mathcal{Y}}_{\mathcal{P}}$ and an appropriate solid torus. Dotted lines indicate $\alpha$ arcs which are fibers. The arrangement on the left changes the fiber from $\alpha_{1}$ to $\alpha_{2}$, while the arrangement on the right does the opposite.

boundary component with the desired $\alpha$ arc as fiber, the fiber direction can be changed as follows:

- To change the fiber from $\alpha_{1}$ to $\alpha_{2}$, extend the bundle by $\mathcal{Y}_{\mathcal{P}}$, attached along the $\rho$ boundary, with the $\sigma$ boundary capped off by a solid torus as in Figure 23 (left).

- To change the fiber from $\alpha_{2}$ to $\alpha_{1}$, extend the bundle by $\overline{\mathcal{Y}}_{\mathcal{P}}$, attached along the $\rho$ boundary, with the $\sigma$ boundary capped off by a solid torus as in Figure 23 (right).

For acyclic graphs any plumbing will work when combining vertices along an edge. In general, however, there is an additional consideration: edges are decorated by a sign, which distinguishes between two plumbing options. In terms of bordered Heegaard diagrams, the difference is between gluing two type $D$ boundaries with fiber $\alpha_{1}$ or gluing two boundaries with fiber $\alpha_{2}$. Suppose we orient each boundary component so that the positive fiber direction is to the left of the positive base direction at a fiberbase intersection. Then a type $D$ boundary with fiber $\alpha_{1}$ has oriented fiber $-\alpha_{1}$ and oriented base $+\alpha_{2}$. Since gluing type $D$ boundaries glues $\alpha_{1}$ to $-\alpha_{2}$, this corresponds to the map $\left(\begin{array}{ll}0 & 1 \\ 1 & 0\end{array}\right)$ in the standard \{base, fiber\} basis. That is, gluing two boundaries with $\alpha_{1}$ fibers corresponds to a + edge. A type $D$ boundary with fiber $\alpha_{2}$ has oriented fiber $+\alpha_{2}$ and oriented base $+\alpha_{1}$, so gluing two of these boundaries corresponds to the map $\left(\begin{array}{rr}0 & -1 \\ -1 & 0\end{array}\right)$.

Once the bundles of two adjacent vertices have been plumbed, the result is no longer an $S^{1}$-bundle. However, continue to keep track of which $\alpha$ arc is the "fiber" at each boundary component. Repeat the process above to add on successive vertices. If at any point an edge connects to a vertex that has already been incorporated, insert the bimodule $\widehat{\mathrm{CFDD}}\left(\mathcal{H}_{\mathrm{SG}}\right)$ and take the appropriate Hochschild homology instead of a tensor product. 


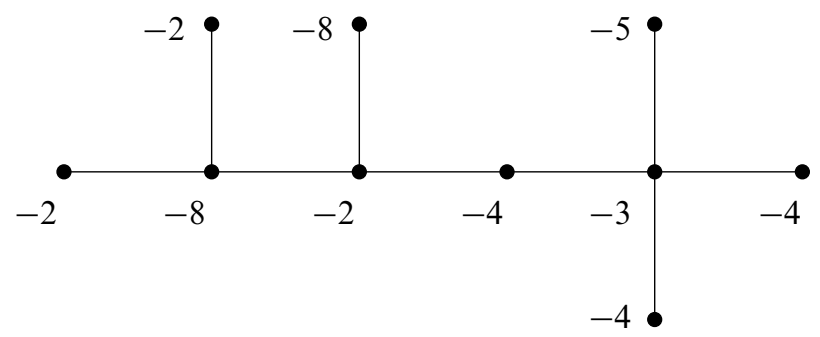

Figure 24: Plumbing graph for a graph manifold with $\operatorname{rk}(\widehat{\mathrm{HF}})=213,312$. The weights on the vertices correspond to Euler numbers; the genus is zero for every vertex and we omit it from the notation.

\section{D Example computations}

The author has implemented a program ${ }^{1}$ using the techniques described above to compute the total rank of $\widehat{\mathrm{HF}}$ of a closed graph manifold, or the bordered invariant of a graph manifold with boundary, from a plumbing graph. It can be used, for example, to see that the rank of $\widehat{\mathrm{HF}}$ of the manifold represented by the negative definite plumbing tree in Figure 24 is 213,312 . It is easy to compute $\left|H_{1}\right|$ from the plumbing graph and see that this manifold is an $L$-space. This is as expected; the fact that this plumbing graph corresponds to an $L$-space follows from [16, Theorem C]. With such large homology, this example is near the limit of the current implementation's reach; the computation took roughly 12 hours to run on a personal computer. For small examples, such as the $E_{8}$ plumbing for the Poincaré homology sphere or small Brieskorn spheres, computations take fractions of a second.

With this algorithm, we can quickly run computations for large sets of graph manifolds and check, for instance, which are $L$-spaces. Consider as an example the plumbing graph $\Gamma$ below, with weights in the range $-5 \leq a, b \leq 5$ and $-5 \leq c, d, e, f \leq-2$ (the bound of -2 on the weights of the outer vertices is so that we only consider graphs in normal form, in the notation of [18]).

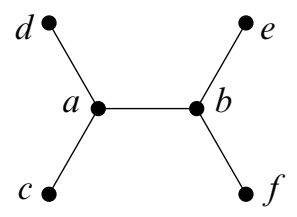

There are 6106 distinct graphs of this form. Of the corresponding 3-manifolds, 5643 are $L$-spaces. Some of these trees are negative definite, but most are not. To

${ }^{1}$ Available at https://github.com/hanselman/HFhat_graph_manifolds. 


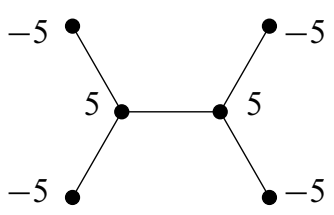

$\operatorname{rk}(\widehat{\mathrm{HF}})=17,600$ $\left|H_{1}\right|=17,600$

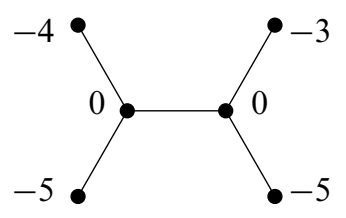

$\operatorname{rk}(\widehat{\mathrm{HF}})=230$

$\left|H_{1}\right|=228$

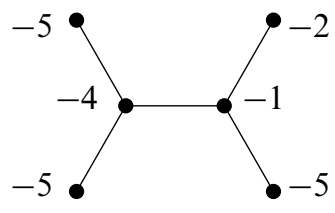

$\operatorname{rk}(\widehat{\mathrm{HF}})=72$

$\left|H_{1}\right|=20$

Figure 25: The manifold corresponding to the graph on the left has the largest $\widehat{\mathrm{HF}}$ of the 6106 examples tested, and it is an $L$-space. The graph in the middle gives the smallest difference between $\mathrm{rk}(\widehat{\mathrm{HF}})$ and $\left|H_{1}\right|$ possible for a non- $L$-space, and the third gives the largest difference among this set of examples.

the author's knowledge, there is currently no other way to compute $\widehat{\mathrm{HF}}$ for these nondefinite examples. Results for a few examples are in Figure 25.

Our final example is the manifold $\Sigma \times S^{1}$, where $\Sigma$ is the surface of genus two. This manifold can be represented by a plumbing graph with just one vertex and no edges. The vertex carries the weights 2 and 0 for the genus and Euler number, respectively. Evaluating the rank of $\widehat{\mathrm{HF}}$ from this graph gives 24, which agrees with the result in [5].

\section{References}

[1] F Bourgeois, Y Eliashberg, H Hofer, K Wysocki, E Zehnder, Compactness results in symplectic field theory, Geom. Topol. 7 (2003) 799-888 MR

[2] S Boyer, C M Gordon, L Watson, On L-spaces and left-orderable fundamental groups, Math. Ann. 356 (2013) 1213-1245 MR

[3] J Hales, D Karabash, M T Lock, A modification of the Sarkar-Wang algorithm and an analysis of its computational complexity, preprint (2007) arXiv

[4] M Hedden, A S Levine, Splicing knot complements and bordered Floer homology, preprint (2012) arXiv

[5] S Jabuka, T E Mark, On the Heegaard Floer homology of a surface times a circle, Adv. Math. 218 (2008) 728-761 MR

[6] A S Levine, Knot doubling operators and bordered Heegaard Floer homology, J. Topol. 5 (2012) 651-712 MR

[7] R Lipshitz, A cylindrical reformulation of Heegaard Floer homology, Geom. Topol. 10 (2006) 955-1097 MR

[8] R Lipshitz, P Ozsváth, D Thurston, Bordered Heegaard Floer homology: invariance and pairing, preprint (2011) arXiv 
[9] R Lipshitz, PS Ozsváth, D P Thurston, Computing $\widehat{H F}$ by factoring mapping classes, Geom. Topol. 18 (2014) 2547-2681 MR

[10] R Lipshitz, PS Ozsváth, DP Thurston, Bimodules in bordered Heegaard Floer homology, Geom. Topol. 19 (2015) 525-724 MR

[11] R Lipshitz, D Treumann, Noncommutative Hodge-to-de Rham spectral sequence and the Heegaard Floer homology of double covers, preprint (2012) arXiv

[12] P Lisca, A I Stipsicz, On the existence of tight contact structures on Seifert fibered 3-manifolds, Duke Math. J. 148 (2009) 175-209 MR

[13] C Manolescu, P Ozsváth, Heegaard Floer homology and integer surgeries on links, preprint (2010) arXiv

[14] C Manolescu, P Ozsváth, S Sarkar, A combinatorial description of knot Floer homology, Ann. of Math. 169 (2009) 633-660 MR

[15] C Manolescu, P Ozsváth, D Thurston, Grid diagrams and Heegaard Floer invariants, preprint (2009) arXiv

[16] M Mauricio, On lattice cohomology and left-orderability, preprint (2013) arXiv

[17] A Némethi, Lattice cohomology of normal surface singularities, Publ. Res. Inst. Math. Sci. 44 (2008) 507-543 MR

[18] W D Neumann, A calculus for plumbing applied to the topology of complex surface singularities and degenerating complex curves, Trans. Amer. Math. Soc. 268 (1981) 299-344 MR

[19] W D Neumann, Graph 3-manifolds, splice diagrams, singularities, from "Singularity theory" (D Chéniot, N Dutertre, C Murolo, D Trotman, A Pichon, editors), World Sci. Publ., Hackensack, NJ (2007) 787-817 MR

[20] P S Ozsváth, A I Stipsicz, Z Szabó, A combinatorial description of the $U^{2}=0$ version of Heegaard Floer homology, Int. Math. Res. Not. 2011 (2011) 5412-5448 MR

[21] P Ozsváth, A I Stipsicz, Z Szabó, Combinatorial Heegaard Floer homology and nice Heegaard diagrams, Adv. Math. 231 (2012) 102-171 MR

[22] P Ozsváth, A Stipsicz, Z Szabó, Knot lattice homology in L-spaces, preprint (2012) arXiv

[23] P Ozsváth, A I Stipsicz, Z Szabó, Knots in lattice homology, Comment. Math. Helv. 89 (2014) 783-818 MR

[24] P Ozsváth, A I Stipsicz, Z Szabó, A spectral sequence on lattice homology, Quantum Topol. 5 (2014) 487-521 MR

[25] P Ozsváth, Z Szabó, On the Floer homology of plumbed three-manifolds, Geom. Topol. 7 (2003) 185-224 MR

[26] P Ozsváth, Z Szabó, Holomorphic disks and knot invariants, Adv. Math. 186 (2004) 58-116 MR 
[27] P Ozsváth, Z Szabó, Holomorphic disks and topological invariants for closed threemanifolds, Ann. of Math. 159 (2004) 1027-1158 MR

[28] P Ozsváth, Z Szabó, Holomorphic triangles and invariants for smooth four-manifolds, Adv. Math. 202 (2006) 326-400 MR

[29] T Peters, On L-spaces and non left-orderable 3-manifold groups, preprint (2009) arXiv

[30] J A Rasmussen, Floer homology and knot complements, $\mathrm{PhD}$ thesis, Harvard University (2003) MR Available at http://search.proquest.com/docview/305332635

[31] S Sarkar, J Wang, An algorithm for computing some Heegaard Floer homologies, Ann. of Math. 171 (2010) 1213-1236 MR

[32] R Zarev, Bordered Floer homology for sutured manifolds, preprint (2009) arXiv

Department of Mathematics, University of Texas at Austin,

1 University Station, C1200, Austin, TX 78712, United States

hanselman@math.utexas. edu

Received: 22 November 2013 Revised: 12 January 2016 